\title{
Carbon Black Reborn: Structure and Chemistry for Renewable Energy Harnessing
}

\author{
Saeid Khodabakhshi, ${ }^{1}$ Pasquale F. Fulvio, ${ }^{2 *}$ Enrico Andreoli ${ }^{1} *$
}

${ }^{1}$ Energy Safety Research Institute, Swansea University, Bay Campus, Swansea SA1 8EN, UK.

${ }^{2}$ Department of Nuclear Engineering, Texas A\&M University, College Station, TX 77843, USA.

\begin{abstract}
Carbon Black (CB) is one of the most abundantly produced carbon nanostructured materials, and approximately $70 \%$ of it is used as pigment and as reinforcing phase in rubber and plastics. Recent scientific findings report on other uses of $\mathrm{CB}$ that are of current interest, such as renewable energy harvesting and carbon capture. The present review focuses on the use and role of $\mathrm{CB}$ in renewable and environmental applications relevant to contemporary global challenges focusing specifically on clean energy. Key and recent research on the structure and chemistry of $\mathrm{CB}$, including its uses as precursors to graphene quantum dots and hollow carbon spheres, is discussed in relation to renewable energy devices, electrochemical energy storage and environmental remediation. The surface chemistry of $\mathrm{CB}$ is closely related to that of graphitic and of turbostratic carbons through the predominant hexagonal carbon lattice from graphene fragments forming its basic structural units. Consequently, modern methods for grafting polymers and functional groups are easily translated to this abundant nanostructured material. Moreover, recent advances in electron microscopy that probe the structure of $\mathrm{CB}$, and its electronic and physicochemical properties in nanocomposites revived the attention of what is wrongfully considered as a scientifically uninspiring material with limited potential for future technology breakthrough. CB has the potential to surge as a key player in renewable energy and environmental applications, meaning "When Black Turns Green".
\end{abstract}


Corresponding Author, Tel: ++1(979)458-8164, E-mail: pfulvio@ @amu.edu \& Tel: (01792)

602524, E-mail: e.andreoli@swansea.ac.uk 

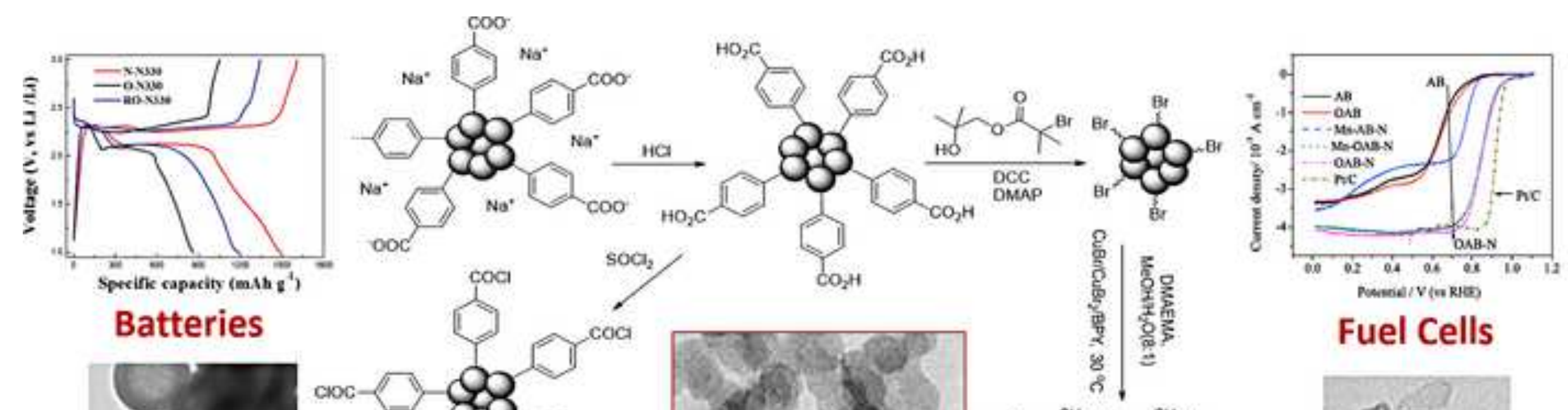

\section{Fuel Cells}

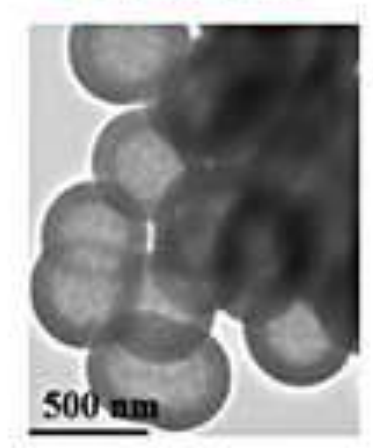

Nanomaterials and

Nanocomposites

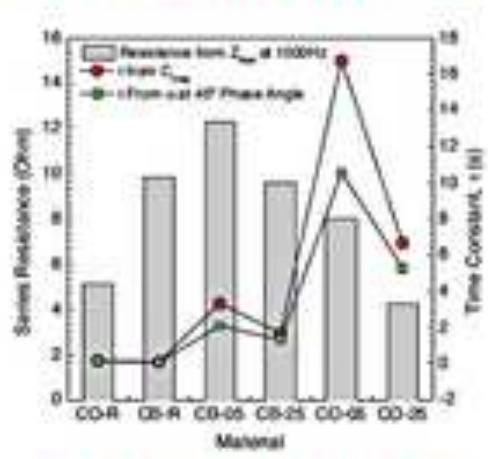

Supercapacitors
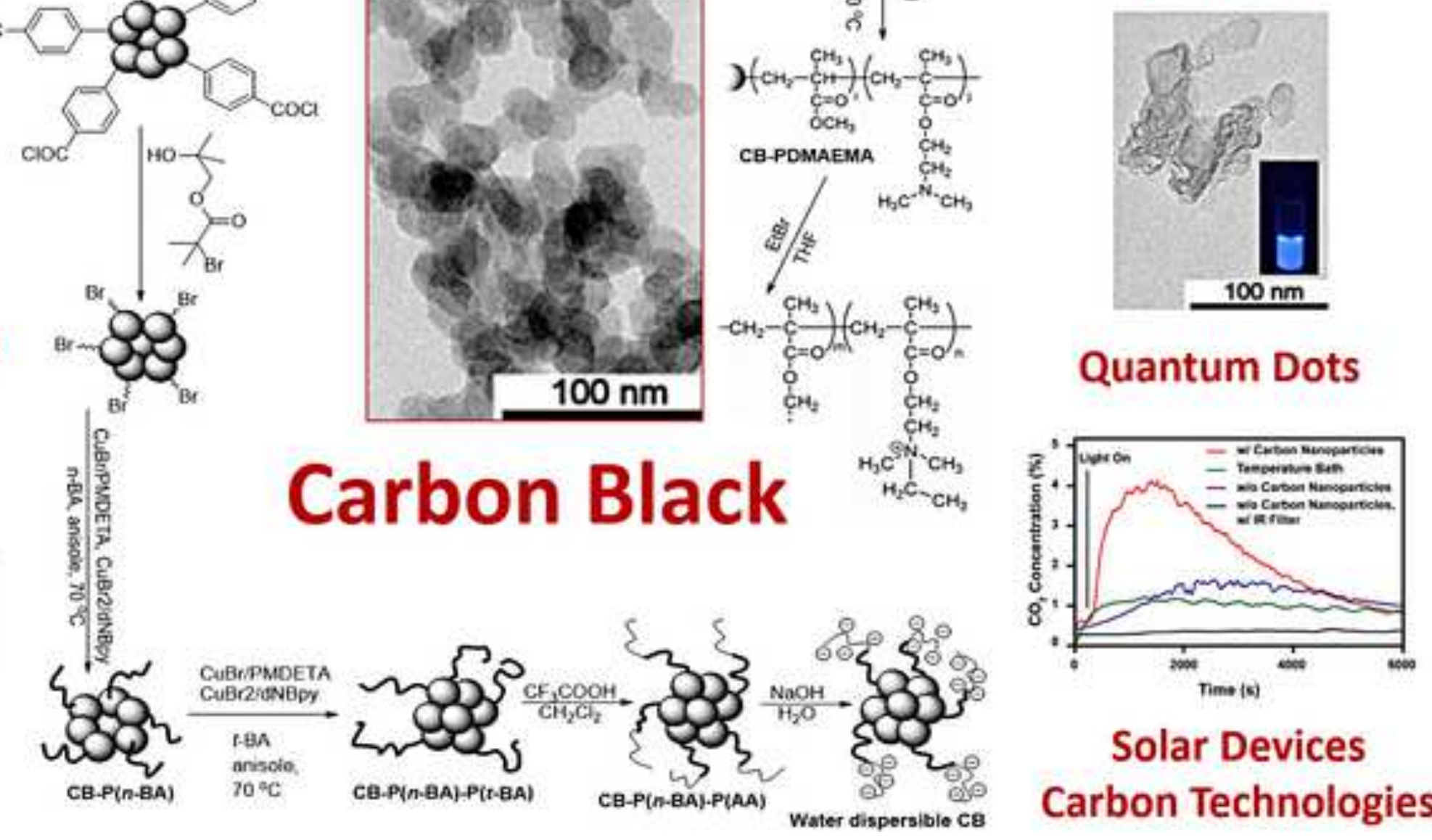

Solar Devices Carbon Technologies 
Table of Contents

Abstract

1 Introduction

2 Carbon black materials

2.1 Particle structure and modification

2.2 Formation of particles and aggregates

\subsection{Alternative production methods}

2.4 Carbon black electrical properties

3 Chemical functionalization

\subsection{Oxidation}

\subsubsection{Gaseous oxidation}

\subsubsection{Wet oxidation}

3.2 Surface modification of oxidized carbon black

3.3 Covalent functionalization by direct carbon-carbon bond formation

3.4 Functionalization with polymers

3.5 Alternative approaches

4 Emerging energy and environmental applications of CB-based materials

4.1 Fuel cells

\subsection{Batteries}

\subsection{Supercapacitors}

4.4. Photocatalysts and Solar Devices

$4.5 \mathrm{CO}_{2}$ storage and separations

5 Conclusions

Abbreviation list

Acknowledgement

References

\section{Introduction}

Carbon black (CB, CAS No. 1333-86-4) materials are formed under the rigorously controlled thermal decomposition of carbon rich feeds in inert (pyrolysis) or oxygen-depleted (partial combustion) atmosphere. Aggregates of carbon nanoparticles (NP) are thus produced, and $\mathrm{CB}$ is in all effects the longest-established commercially-marketed form of carbon 
nanostructured material. CB production process is fundamentally different from that of black carbon and soot, both resulting from the incomplete non-purposely engineered combustion of carbon sources such as fossil fuels, biofuels, and biomass. CB is the cheapest and largest scale industrially produced nanocarbon with a projected annual production of 15 Mt by 2025 [1]. CB dwarfs the market of carbon nanotubes (15 kt by 2022 [2]) and with an average price of $\$ 1 / \mathrm{kg}$, it is by far cheaper than the cheapest grade of carbon nanotubes currently available on the market, $\$ 600 / \mathrm{kg}$ [3]. To further exacerbate this contrast, graphene nanoplatelets and fullerene $\mathrm{C}_{60}$ are sold at about $\$ 10,000 / \mathrm{kg}$ and $\$ 35,000 / \mathrm{kg}[4,5]$, respectively. Hence, where large scale availability, cost of materials, and required properties meet those of other carbon allotropes, CB appears to be an attractive option.

Impingement, gas, lamp, channel, furnace, thermal, and acetylene blacks are all different types of $\mathrm{CBs}$, each produced using different processes and/or starting materials. Currently, the vast majority of CBs are furnace blacks, while some others are thermal and acetylene blacks. The other CBs have been phased out because their preparation processes were less economically viable or less environmentally friendly [6]. The furnace black process can produce practically all grades of $\mathrm{CB}$ used in the rubber industry, the major user of $\mathrm{CB}$ as a reinforcing filler in tires. Key to the production process is a partial combustion furnace where an atomized spray of aromatic oils (rich in polycyclic aromatic hydrocarbons) and an airdeficient fuel source (natural gas or oil) are injected to combust and produce the temperature $\left(1200-1900{ }^{\circ} \mathrm{C}\right)$ required to pyrolyze and covert the aromatic oils into carbon black. Furnace black is freshly collected as a very low bulk density powder (20-60 g/L), compacted or pelletized to facilitate handling [6]. Thermal blacks are produced in an inert atmosphere using lower molecular weight gaseous hydrocarbons. A furnace is heated to the required temperature $\left(1400{ }^{\circ} \mathrm{C}\right)$ with the combustion of natural gas in air, at that temperature the air flow is stopped letting in only gas that is then pyrolyzed to $\mathrm{CB}$ and $\mathrm{H}_{2}$. Acetylene black production is much smaller in scale since acetylene is an expensive feed material, and it is only used in specialized high-value applications most often as (electrical) conductivity and battery blacks.

Typically, furnace blacks have specific surface areas from 20 to $150 \mathrm{~m}^{2} / \mathrm{g}$, but, values up to $300 \mathrm{~m}^{2} / \mathrm{g}$ can be achieved in the case of pigment-grade furnace blacks. Rubber-grade furnace blacks can contain significant amounts of oxygen, ash, and volatiles with a resulting carbon content not lower than 97\% [7]. Ash residues are usually due to salts present in the water used to quench the pyrolysis process, or purposely added to control the $\mathrm{CB}$ structure. 
Hydrogen, nitrogen and sulfur elements can also be found in $\mathrm{CB}$ depending on the raw material feeds used. C-H hydrogen is found at the edge of the carbon layers, while nitrogen and sulfur are usually integrated in the aromatic structure of the layer. Oxygen groups such as $\mathrm{COOH}, \mathrm{C}=\mathrm{O}$, and $\mathrm{OH}$ are present on the surface depending on the use of air during the production process. Thermal and acetylene blacks instead consist of $>99 \%$ carbon [7]. Some thermal black grades can be made very coarse with specific surface areas of a few $\mathrm{m}^{2} / \mathrm{g}$, while some pigment grades can be powders with specific surface areas of a thousand $\mathrm{m}^{2} / \mathrm{g}$ [6]. CBs are indeed a very wide family of materials as exemplified by the number of commercial products available on the market covering an as wide variety of technological specifications and performance requirements. Examples of key global producers with some of their main product trademarks and series are: Cabot Corporation (BLACK PEARLS®, EMPEROR®， MONARCH®， REGAL®， VULCAN®)， Birla Carbon （RAVEN®, CONDUCTEX®, ULTRA®, BC and N series), Continental Carbon (N, CL, E, JE, LH, Satin, and SBX series), Orion Engineered Carbon (CORAX®, PUREX®, DUREX®, NEROX®, PRINTEX®), and Imerys Graphite \& Carbon (Super $\mathrm{S} \AA$, Super P®, ENSACO ${ }^{\circledR}$ ). A summary of the most referenced CB materials and some of their properties, which are discussed in the sections herein, are included in Table 1. Selected CB materials, properties and applications have been compiled from the representative references cited in this work. The table includes materials not mentioned in the present text, but that were extracted from the same references as those explicitly mentioned in the examples. ASTM International (formerly known as American Society for Testing and Materials) reference numbers were obtained from different vendors and manufacturers whenever a given property or specific application was unavailable from referenced works. A comprehensive list of ASTM nomenclature, numbers and $\mathrm{CB}$ properties is available from Report 93 of the International Agency for Research on Cancer (IARC) [8].

The aim of this document is to review the most significant and latest uses of $\mathrm{CB}$ in applications relevant to current global challenges focusing specifically on clean energy. The different types of $\mathrm{CB}$ grades, structures, properties, manufacturing processes and major industrial applications (e.g. tires, pigments, inks, etc.) are already covered in numerous excellent chapters and reviews [6, 7, 9-12]. Therefore, our focus is on recent work covering these different aspects of $\mathrm{CB}$ and only revisiting some of the general knowledge where beneficial. A significant part of this review is also dedicated to the chemistry of CB since chemical modification and functionalization are often instrumental to application. Solar cells, 
fuel cells, batteries, and supercapacitors are the key applications discussed in this review, with an additional section dedicated to $\mathrm{CB}$ applied to carbon dioxide capture also relevant to the deployment of low carbon energy technologies. 
Table 1 - Most commonly reported Carbon Black materials and discussed in the present work, properties and uses.

\begin{tabular}{|c|c|c|c|c|c|c|c|c|}
\hline Manufacturer & $\begin{array}{c}\text { CB } \\
\text { Grade }\end{array}$ & $\begin{array}{c}\text { Commercial } \\
\text { Name } \\
\text { (ASTM \#) }\end{array}$ & Type & $\begin{array}{l}\text { PPS } \\
(\mathrm{nm})\end{array}$ & $\begin{array}{l}\text { SSA } \\
\left(\mathrm{m}^{2} / \mathrm{g}\right)\end{array}$ & Functionalization & Uses & $\begin{array}{c}\text { Representative } \\
\text { References }\end{array}$ \\
\hline & & BP120 & & $\begin{array}{l}52- \\
60\end{array}$ & 30 & \multirow{7}{*}{ Oxidation } & & \multirow{7}{*}[40,41]{} \\
\hline & & BP280 & & 34 & 41 & & Conductive & \\
\hline & BLACK & BP460 & Furnace & 29 & 78 & & additives, fuel & \\
\hline & PEARLS $^{\circledR}$ & BP800 & Black & 22 & 242 & & cells, carbon & \\
\hline & & BP880 & & 19 & 210 & & capture & \\
\hline Cabot & & BP1300 & & 13 & 520 & & & \\
\hline \multirow[t]{5}{*}{ Corporation } & & BP2000 & & 15 & 1500 & & & \\
\hline & \multirow{4}{*}{ VULCAN $^{\circledR}$} & $\begin{array}{l}\text { XC-72R, } \\
\text { powder }\end{array}$ & & $\begin{array}{l}20- \\
50\end{array}$ & 241 & \multirow{4}{*}{$\begin{array}{c}\text { Oxidation, } \mathrm{C}-\mathrm{N} \\
\text { coupling, } \mathrm{C}-\mathrm{C} \\
\text { coupling through } \\
\text { radical reactions } \\
\text { or by diazonium } \\
\text { salts }\end{array}$} & \multirow{4}{*}{$\begin{array}{c}\text { Conductive } \\
\text { additives, fuel } \\
\text { cells, } \\
\text { supercapacitors }\end{array}$} & \\
\hline & & $\begin{array}{c}\text { XC-72, } \\
\text { pellet }\end{array}$ & Furnace & $\begin{array}{l}30- \\
60\end{array}$ & 218 & & & $\begin{array}{c}{[169,171,} \\
176,177,180,\end{array}$ \\
\hline & & $\mathrm{P}(\mathrm{N} 294)$ & Black & $\begin{array}{l}20- \\
25\end{array}$ & 140 & & & $\begin{array}{c}185-188,194- \\
197,231]\end{array}$ \\
\hline & & $9(\mathrm{~N} 115)$ & & $\begin{array}{l}11- \\
19\end{array}$ & 123 & & & \\
\hline
\end{tabular}




\begin{tabular}{|c|c|c|c|c|c|c|c|c|}
\hline \multirow{14}{*}{ 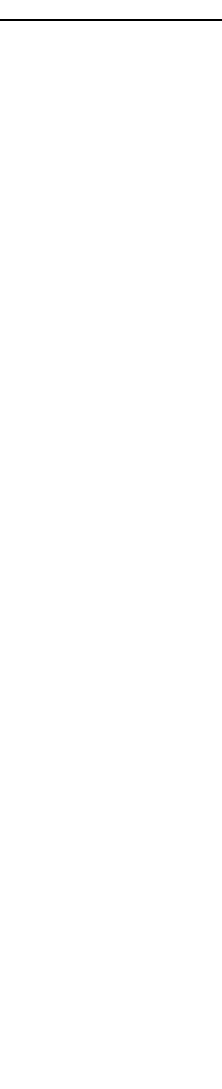 } & & \multicolumn{2}{|r|}{$\begin{array}{c}\text { Acetylene } \\
\text { Black }\end{array}$} & 34 & 254 & & & \\
\hline & \multirow{8}{*}{ MONARCH ${ }^{\circledR}$} & 120 & & 75 & 31 & Oxidation, C-N & & \multirow{8}{*}[135,144-148]{} \\
\hline & & 430 & & 30 & 85 & coupling, C-C & & \\
\hline & & 700 & & 18 & 200 & coupling through & & \\
\hline & & 800 & Furnace & 19 & 210 & atom transfer & & \\
\hline & & 1100 & Black & 14 & 240 & radical & & \\
\hline & & \multirow{3}{*}{1300} & & \multirow{3}{*}{13} & \multirow{3}{*}{560} & polymerization & & \\
\hline & & & & & & (ATRP) or by & & \\
\hline & & & & & & diazonium salts & & \\
\hline & \multirow{2}{*}{ MOGUL $^{\circledR}$} & \multirow[b]{2}{*}{$\mathrm{L}$} & Furnace & \multirow[b]{2}{*}{21} & \multirow[b]{2}{*}{138} & Oxidation, C-N & & \multirow[b]{2}{*}[38]{} \\
\hline & & & & & & coupling & & \\
\hline & \multirow{3}{*}{ REGAL $^{\circledR}$} & 250 & \multirow{3}{*}{$\begin{array}{c}\text { Furnace } \\
\text { Black }\end{array}$} & 35 & 78 & & Conductive & \multirow{3}{*}[16,125,171]{} \\
\hline & & 400 , pellet & & 46 & 115 & Oxidation & additives, fuel & \\
\hline & & $660 \mathrm{R}$ & & 21 & 121 & & cells & \\
\hline \multirow{4}{*}{$\begin{array}{l}\text { Orion } \\
\text { Engineered } \\
\text { Carbon }\end{array}$} & \multirow{4}{*}{ CORAX $^{\circledR}$} & N110 & & $\begin{array}{r}11- \\
19\end{array}$ & 130 & & Nanofluids, & \multirow{4}{*}{$\begin{array}{c}{[104,138,} \\
161,269,270, \\
276]\end{array}$} \\
\hline & & N115 & Furnace & $11-$ & \multirow[t]{2}{*}{137} & & Solar energy & \\
\hline & & \multirow[b]{2}{*}{ N121 } & DTack & 19 & & & conversion, & \\
\hline & & & & $\begin{array}{c}11- \\
19\end{array}$ & 114 & & carbon capture & \\
\hline
\end{tabular}




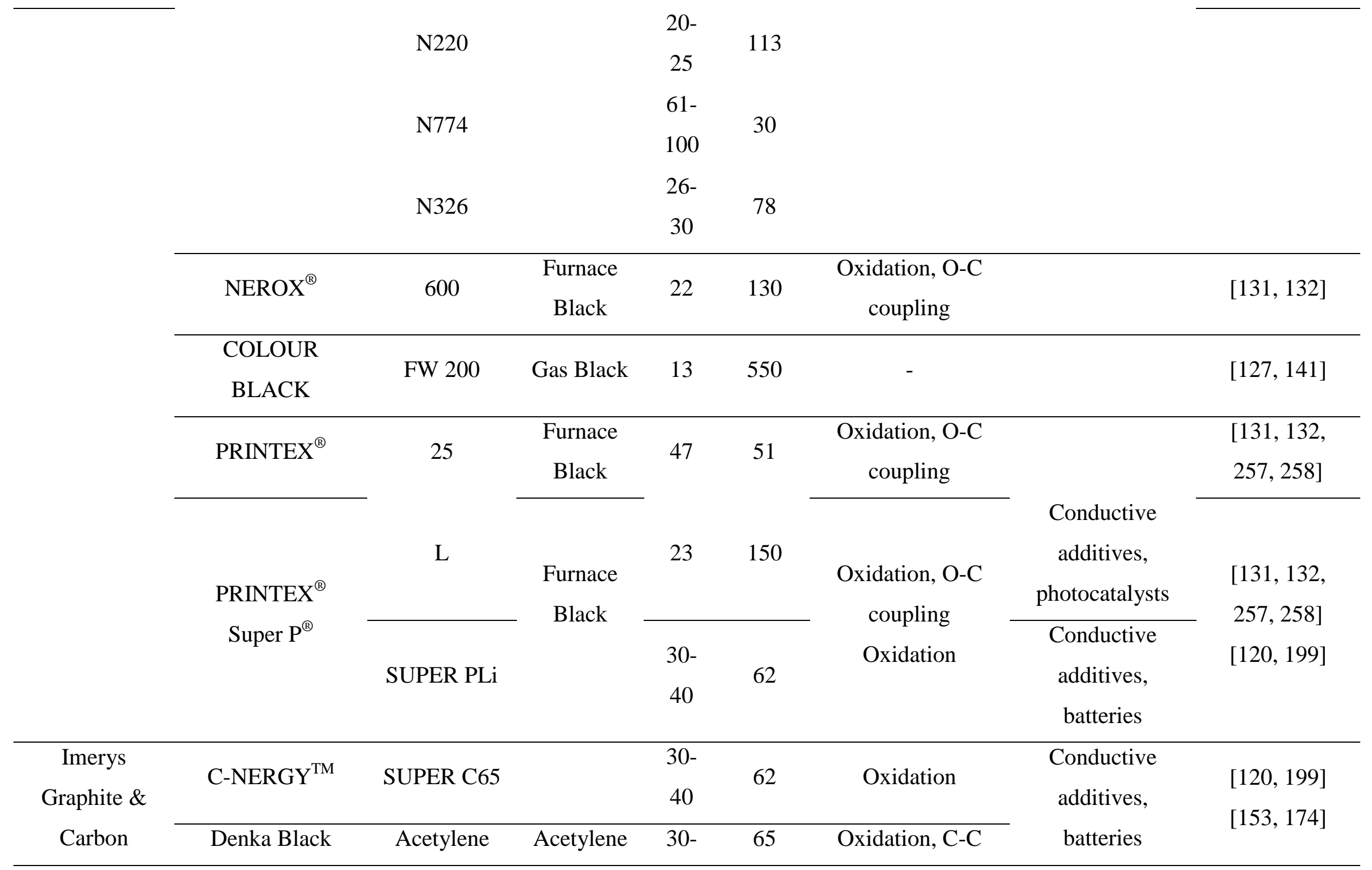




\begin{tabular}{|c|c|c|c|c|c|c|c|c|}
\hline & (DB) & Black & Black & 40 & & $\begin{array}{l}\text { coupling through } \\
\text { radical reactions }\end{array}$ & $\begin{array}{c}\text { Conductive } \\
\text { additives, fuel } \\
\text { cells, } \\
\text { supercapacitors }\end{array}$ & \\
\hline $\begin{array}{c}\text { Denka } \\
\text { Corporation }\end{array}$ & Ketjenblack $^{\circledR}$ & $\begin{array}{l}\text { EC300J, } \\
\text { granule }\end{array}$ & $\begin{array}{c}\text { Acetylene } \\
\text { Black }\end{array}$ & 79 & 800 & Oxidation & $\begin{array}{c}\text { Conductive } \\
\text { additives, fuel } \\
\text { cells, } \\
\text { supercapacitors, } \\
\text { carbon capture }\end{array}$ & {$[44,47,115]$} \\
\hline \multirow[b]{2}{*}{ AkzoNobel } & & $\begin{array}{l}\text { EC600JD, } \\
\text { powder }\end{array}$ & \multirow[b]{2}{*}{$\begin{array}{c}\text { Acetylene } \\
\text { Black } \\
\text { Acetylene } \\
\text { Black }\end{array}$} & 68 & 1270 & \multirow[b]{2}{*}{$\begin{array}{l}\text { Oxidation } \\
\text { Oxidation }\end{array}$} & $\begin{array}{l}\text { Conductive } \\
\text { additives, fuel }\end{array}$ & \\
\hline & $\begin{array}{c}\text { Ketjenblack }^{\circledR} \\
\text { Shawinigan } \\
\text { Black }^{\circledR}\end{array}$ & $\mathrm{C}-55$ & & 42 & 70 & & $\begin{array}{c}\text { cells, } \\
\text { supercapacitors, } \\
\text { carbon capture } \\
\text { Conductive } \\
\text { additives, fuel } \\
\text { cells, batteries }\end{array}$ & $\begin{array}{r}{[44,47,115]} \\
{[169,174]}\end{array}$ \\
\hline $\begin{array}{l}\text { Chevron } \\
\text { Phillips }\end{array}$ & Neo-Spectra & Neo-Spectra & $\begin{array}{c}\text { Channel } \\
\text { Black }\end{array}$ & 25 & 1000 & & & $\begin{array}{c}{[153,226,} \\
235]\end{array}$ \\
\hline $\begin{array}{l}\text { Columbian } \\
\text { Carbon Co. }\end{array}$ & Neo-Spectra & $\begin{array}{c}\text { Neo-Spectra } \\
\text { II }\end{array}$ & $\begin{array}{c}\text { Channel } \\
\text { Black }\end{array}$ & 13 & 906 & $\begin{array}{l}\text { Oxidation, } \mathrm{C}-\mathrm{C} \\
\text { coupling through }\end{array}$ & & $\begin{array}{c}{[153,226,} \\
235]\end{array}$ \\
\hline
\end{tabular}




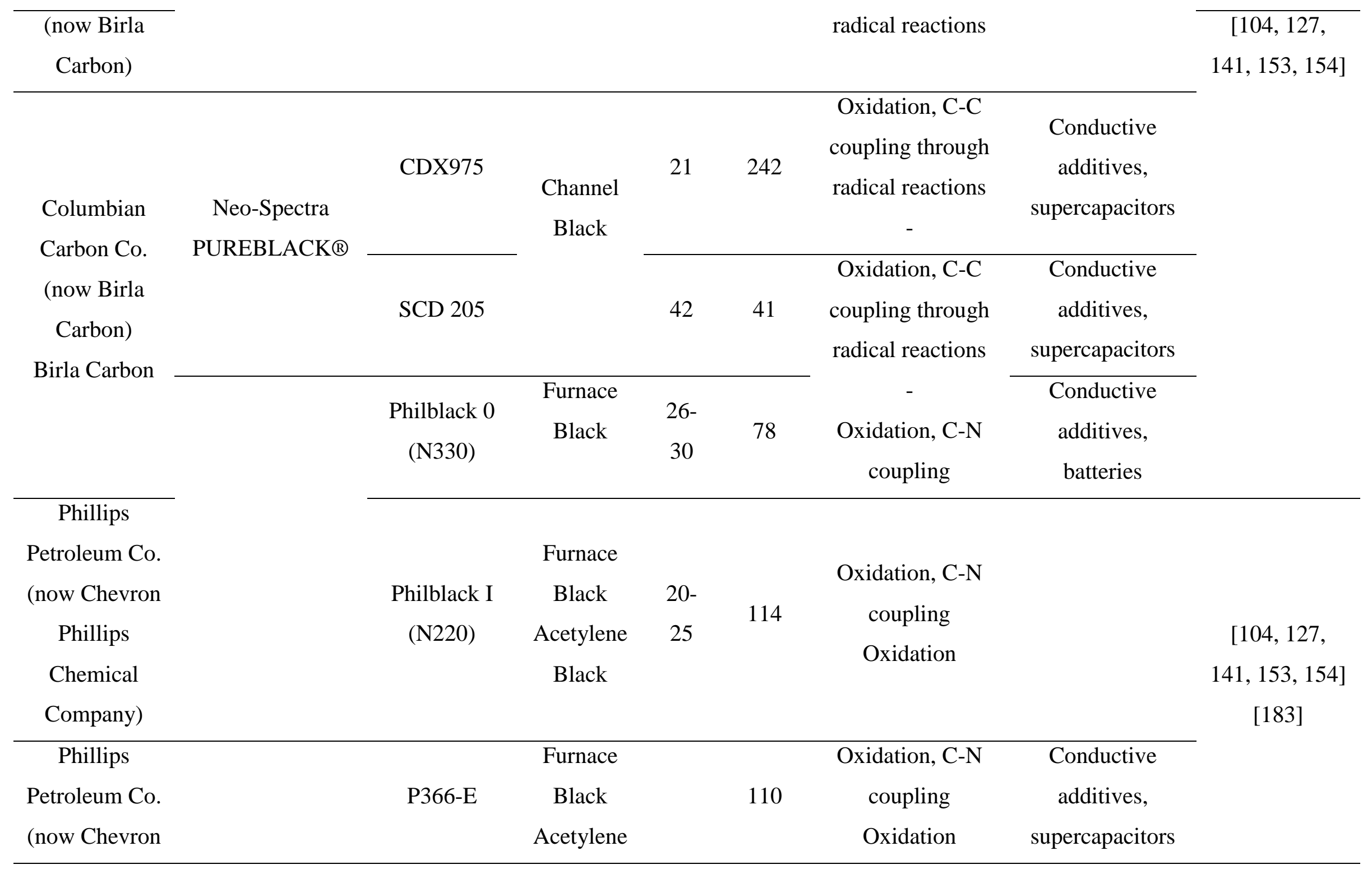




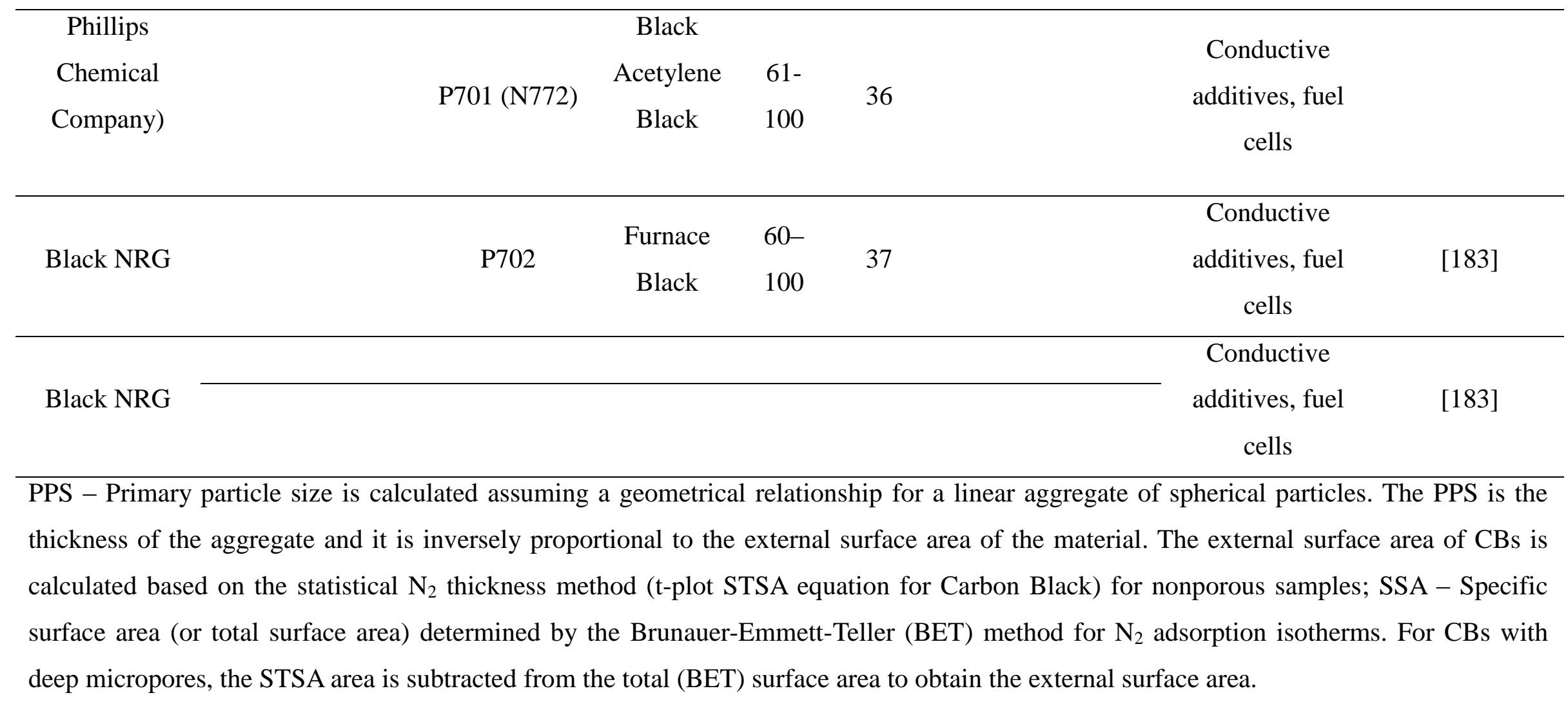




\section{Carbon black materials}

$\mathrm{CBs}$ are engineered to meet specific process and application requirements. Their properties must meet processability specifications (e.g., high dispersibility in rubber to make tires, or in water to make inks), and final product specifications (e.g., high abrasion resistance in tires, or blue undertone in inks). The key material features that determine these properties are down to the particle level where size, porosity, structure, and surface chemistry determine dispersibility, conductivity, rheology, pigmentation, abrasion and UV stabilization, to name some.

\subsection{Particle structure and modification}

CB materials are aggregates of nanoparticles of spheroidal shape, typically 10 to $100 \mathrm{~nm}$ in diameter, with an amorphous core surrounded by a shell of stacked graphene-like domains each made of a nanometric stack of polyaromatic sheets (Figure 1). These domains tend to orient concentrically around the amorphous core forming a disordered onion-like nanostructured shell and are often referred to as basic structural unit (BSU) [13]. Each nanoparticle, also referred to as primary particle, is a non-discrete component of an aggregate, meaning it can only be separated from the other particles by fracturing [14]. The size and number of particles present in an aggregate define its structure: low structure CB has aggregates of spherical shape often made of less and larger particles (coarse structure), whereas high structure $\mathrm{CB}$ has aggregates of branched shape often made of more and smaller particles (fine structure). CB may contain chemically bound hydrogen, oxygen, nitrogen and sulfur depending on the raw materials and production process used. Heteroatoms are removed upon thermal treatment at high temperature in inert atmosphere (carbonization). Increased size of two-dimensionally ordered domains and formation of three-dimensional staking order (graphitization) can be observed above $2000{ }^{\circ} \mathrm{C}$, whereas the amorphous core is at best converted to a microporous structure consisting of warped carbon sheets at a temperature of $3000{ }^{\circ} \mathrm{C}$ and above. Combined X-ray diffraction (XRD), high resolution transmission electron microscopy (HRTEM), and Raman studies showed that $\mathrm{CB}$ is comprised of small sized graphene domains with limited number of stacked layers and greater degree of stacking disorder between parallel planes than graphite. As Pawlyta et al. stated "during graphitization the size of stacked polyaromatic layers cannot exceed the volume inside which the layers were previously oriented in parallel" [15]. This is exemplified in Figure 2 where upon heat treatment at $2600{ }^{\circ} \mathrm{C}$, PRINTEX ${ }^{\circledR} 25$ with larger primary particles allows for the growth of bigger domains resembling the graphitic structure 
compared to Color Black FW200 where stacks of only a few graphene layers are formed. Consequently, CB is generally categorized as a non-graphitizable material since its mismatch stacking (turbostratic structure) carbon intraplanar spacing of $0.344 \mathrm{~nm}$ does not reduce to $0.335 \mathrm{~nm}$ for graphite even after heat treatment at $3000{ }^{\circ} \mathrm{C}$.[16] The latter happens for two main reasons: the small particle size and lattice mismatch. In case of small particle size, the forces binding each stacked graphene domain are weaker than in much larger sheets; the lower interplanar forces results in increased separation between sheets. The second reason results from the inability of each individual sheet to rotate and orient itself with respect to the next two sheets as in graphite. Consequently, the expected ABABA... stacking is not achieved. In the hexagonal graphite unit cell, half of the $\mathrm{C}$ atoms find themselves immediately above and below the centers of hexagons of the adjacent graphene planes. In case of stacking disorders, there is no regular correspondence between $\mathrm{C}$ atoms in adjacent parallel layers, which further increases the interplanar spacing in CB. Instead of forming infinitely large graphitic domains at high temperature, the BSUs flatten out to join other BSUs at high angles, an orientation imposed by the CB particle geometry and size. This junction takes place at the terminations of the edge planes to form closed polyhedrons with layer spacing greater than that of graphite.[16]

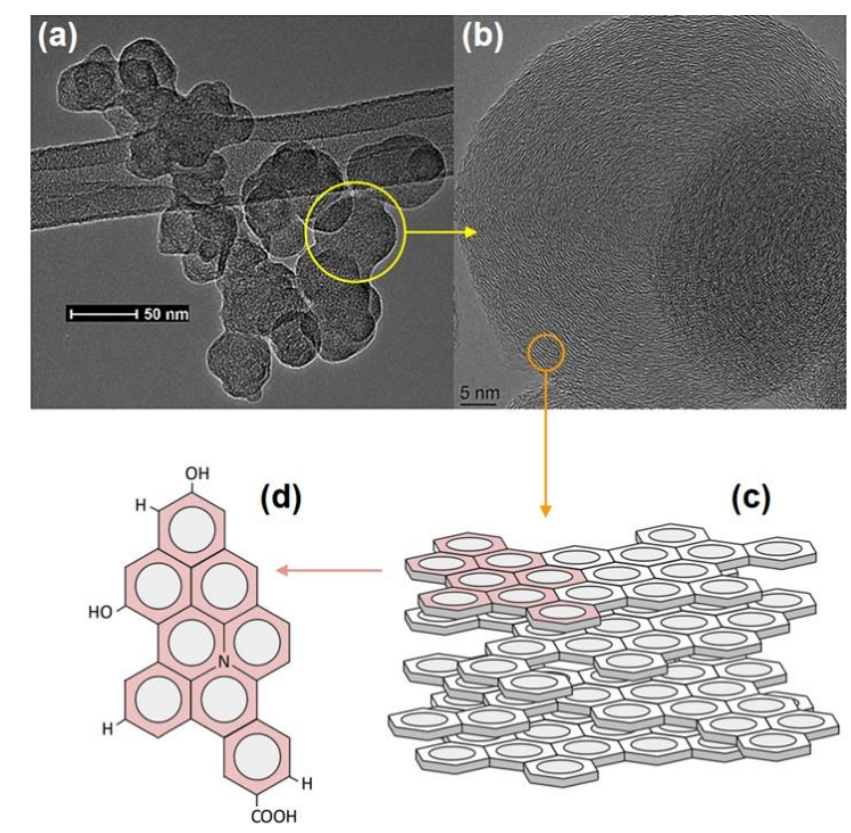

Figure 1 - TEM images of (a) aggregate and (b) primary particle of carbon black Cabot REGAL® 250 (adapted with permission from reference [16]). Illustrative representation of 
(c) a hypothetical graphitic basic structural unit, and (d) a simplified example of hydrogen and heteroatoms present in it.
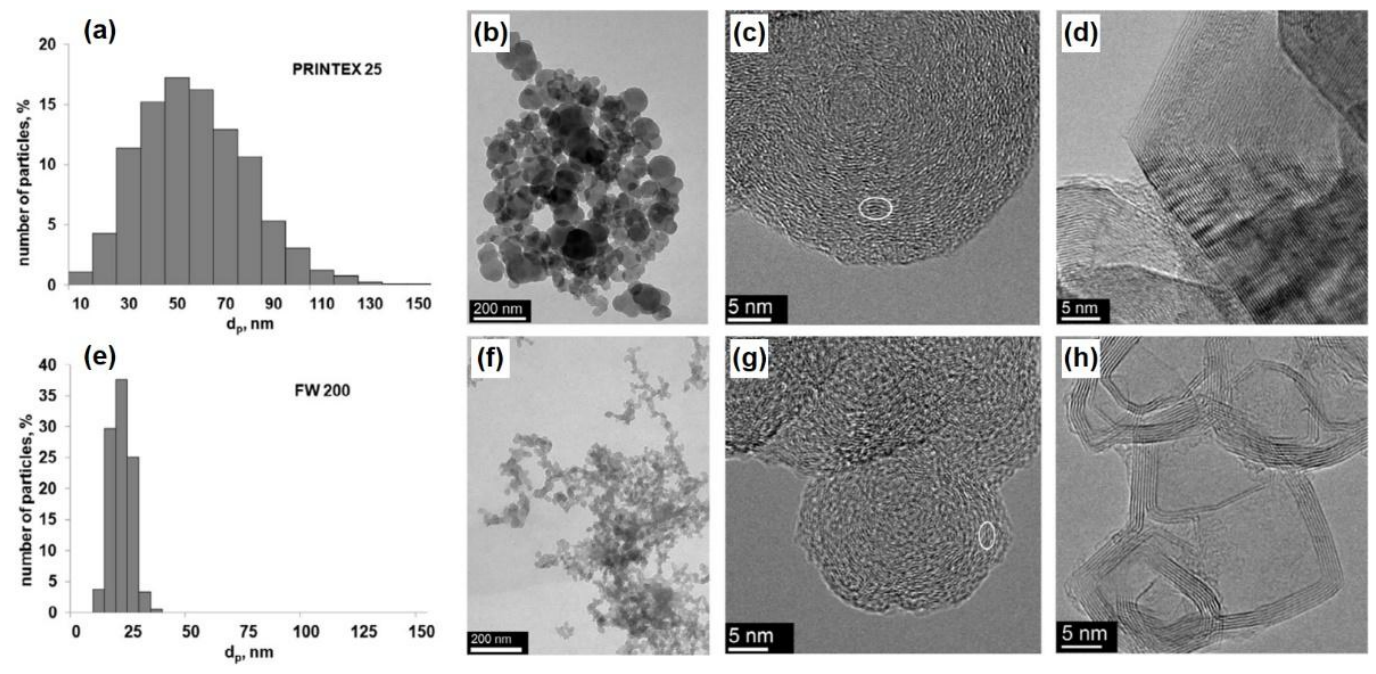

Figure 2 - Effect of heat treatment on two CBs of different particle size distribution: PRINTEX® 25 (a-d) and Color Black FW200 (e-h) both from Orion Engineered Carbons. Distributions of primary particles diameters are in (a) and (e). TEM images of aggregates in (b) and (f), and HRTEM images of primary nanoparticles in (c) and (g), all before heat treatment. HRTEM images of primary particles after heat treatment in argon at $2600{ }^{\circ} \mathrm{C}$ are given in (d) and (h). Examples of BSU are circled in (c) and (g). Reproduced and adapted from reference [15].

A molecular model of the core-shell structure of CB primary particles has been developed by Ban et al. [17], showing small size graphite sheets randomly arranged in the amorphous core and larger size sheets oriented almost concentrically in the shell (Figure 3). The model indicates that larger shell units allow for a better concentric orientation of the carbon layers in the outer shell, with $50 \%$ of all graphene fragments forming BSUs made from the stacking of 2-5 layers. The calculated BSU size were in agreement with those measured experimentally of 1.1-2.0 nm height and 2.0-4.0 nm extent for units of 3-6 parallel layers [17]. The separated core-shell structure of $\mathrm{CB}$ is strikingly evident in samples produced by laser-induced combustion, as shown in Figure 3 (c). 

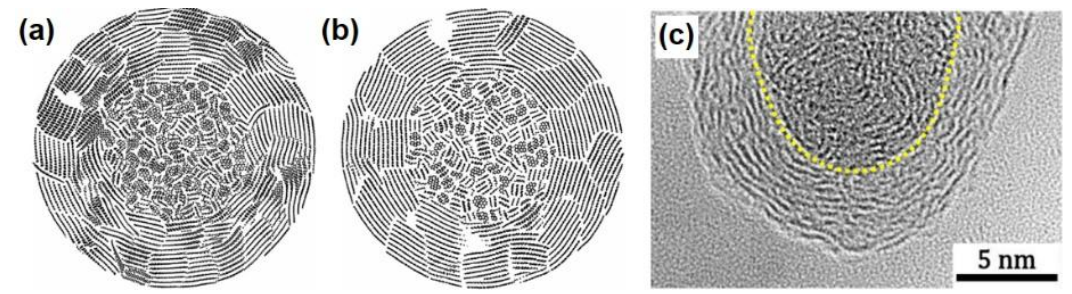

Figure 3 - Slice views of modeled carbon black primary particles with shell units of size 2.7 $\mathrm{nm}$ (a) and $3.7 \mathrm{~nm}$ (b). TEM image of a carbon black particle produced by laser-induced combustion with a yellow dotted line marking the boundary between a disorder core and more organized outer shell. Reproduced and adapted from references [17, 18].

As previously discussed, a BSU domain can be thought as made of a stack of few and small graphene fragments. In addition to flat graphene domains, curved graphene layers are present [19]. Recent HRTEM studies on soot confirmed the presence of curved graphene domains resembling those of fullerene [20]. Such domains in soot also have strong resemblance to those found in CB. The visualization and modeling of these domains on soot provided support to the hypothesis of fullerene-type nucleation during soot and CB growth [21]. The proposal of pentagons into the growth mechanism of soot was driven by the need to eliminate dangling bonds in the growing carbon network. Although topological models indicate that concentric fullerene structures would have $\mathrm{d}_{002}$-spacing that is smaller than those found in soot and CB [22], the existence of carbon pentagons provides curvature to fullerene and to CNT, whereas lines of pentagons give graphene curvature when forming graphitizing porous carbons [23]. Charged fullerene fragments are also known to occur in flames simultaneously to soot particles [24], and $\mathrm{C}_{60}$ was originally observed on graphitized CB [25]. Hence, the presence of such pentagonal defects breaking the periodical hexagonal ordering of planar graphene fragments contributes to curvature and misalignment of curved fragments in soot and in CB [26, 27]. In addition to pentagonal defects in the graphene lattice, other structural defects cannot be ruled out when considering lattice mismatches and curvature, e.g. heptagonal defects and junctions of pentagonal and heptagonal rings [28]. While series of pentagonal rings introduce greater curvature to the graphene fragments that act as building blocks, heptagonal rings reduce such curvature when in contact with a pentagonal defect. Also supporting of the fullerene building blocks, and by analogy to the well-characterized pentagons in fullerenes, models suggest that the fullerene-like structure of $\mathrm{CB}$ has much greater electron affinity than $\mathrm{C}_{60}$ [29]. The consequence of that greater affinity is that the fullerene-like structures may act as free-radical acceptors. Thus, pentagons may play key-role 
in surface grafting of polymers and rubber [29]. Further studies incorporating heptagons and other lattice defects may further refine such models for the reactivity and curvature of $\mathrm{CB}$, and potentially help elucidate the growth mechanism of these materials.

Size and extension of the stacked graphene shell can be amplified upon bombardment with high energy electrons. Pulsed irradiation leads to the reordering of graphene layers [30, 31], whilst continued irradiation with relativistic electrons $(11.2 \mathrm{~kW}$ of power for $7 \mathrm{~min})$ have been shown to induce the formation of nanocapsules with significant increase in the degree of crystallinity [32]. A comparison of CB before and after irradiation is given in Figure 4. The starting material was a thermal $\mathrm{CB}$ with $250 \mathrm{~nm}$ average diameter particles and 0.4-2.0 $\mathrm{nm}$ graphene layers spaced by about $0.40 \mathrm{~nm}$. Upon irradiation, nanocapsules made of graphene layers spaced by $0.355 \mathrm{~nm}$ were formed, Figure 4(e). The increase of crystallinity was evident in the fast Fourier transform of the transmission electron microscopy (TEM) images with more and better-defined electron diffraction spots, see Figures 4(b) and 4(f). Electron bombardment induced fast heating and corresponding conversion of $\mathrm{sp}^{3}$ to $\mathrm{sp}^{2}$ carbon [32], and fast heating is believed to be the cause of turbostratic stacked graphene formation upon high energy electrons bombardment. Similar conversions were observed when high energy laser was used, and turbostratic stacks of extended graphene layers formed by treating $\mathrm{CB}$ with high energy laser reaching temperatures as high as $4200{ }^{\circ} \mathrm{C}$ (Figure 5) [33].

Depending on the energy of the electron beam (e-beam), structural changes or damage to the carbon nanomaterials may undergo different mechanisms. Absorption of electrons in the range of $100 \mathrm{keV}$ to $10 \mathrm{MeV}$ leads to production of secondary electrons via energy loss and collision processes, further producing ions and free radicals in the target material. Hence, the overall effect of the electron bombardment is related to the energy loss due to electron scattering and its dissipation as heat $[34,35]$. In carbon materials, e.g. C60, graphite, SWNT, MWNT and carbon nanofibers, the radiation damage starts with the knock-on process, followed by vacancy interstitials and Stone-Wales (SW) defects and recombination process [36]. A Stone-Wales defect involves the change of connectivity between two $\pi$-bonded C atoms and (this is the replacement of four hexagons in the graphene sheet with two pentagons and two heptagons. This change results from rotation by $90^{\circ}$ of the two $\pi$-bonded $\mathrm{C}$ atoms with respect to the midpoint of their bond [37]. The electron bombardment induces vacancies by dangling bond saturation. This process allows for the welding of SWNT, MWNTS and carbon fibers $[34,38]$. Besides SW defect, Frenkel pairs have recently been pointed out as 
relevant to high-energy e-beam irradiation of carbon nanostructures [39, 40]. Frenkel pairs are a type of point defects, and form when a small atom or ion migrates and leaves a lattice vacancy; this atom or ion then becomes an interstitial in the lattice. Computational methods have further proposed formation of energetically favored Frenkel pairs and bridging neighboring graphene layers in graphite and in materials having curved graphene lattices, e.g. MWNTs, fullerenes and onion-like carbons [36, 40]. In radiation damaged graphite, part of the SW defects has been found to be corrected by Frenkel pairs, with the number of Frenkel pairs becoming more relevant at high energies. This concerted mechanism has been found to restore graphite lattice in radiation exposed graphite, thus rendering it high radiation stability [36]. Moreover, energy is dissipated well-below the surface of a material, and the energy dissipation in the form of heat becomes increasingly important at increasingly higher e-beam energies. The dissipation of the e-beam energy in a massive target (meaning a large volume of a material) has been found to vary with depth. For example, the energy dissipation as heat in $\mathrm{Cu}$ has been investigated experimentally and the maximum energy dissipation rate found to occur at $400 \mathrm{~nm}$ and $600 \mathrm{~nm}$ from the surface for a $20 \mathrm{keV}$ and $30 \mathrm{keV}$ e-beam, respectively. These results indicate that the effects of electron irradiation will be dissipated at a greater depth from the surface of a target material [35]. Although turbostratic and graphitic carbons are both subjected to similar radiation damage mechanisms leading to rearrangement of $\mathrm{sp}^{2} \mathrm{C}$ lattice, intense local heating may further explain the formation of large $\mathrm{CB}$ aggregates $[32,33]$. As pointed by authors investigating SWNTs, e-beam heating induced carbon sublimation, leading to SWNT thickening and welding [38].
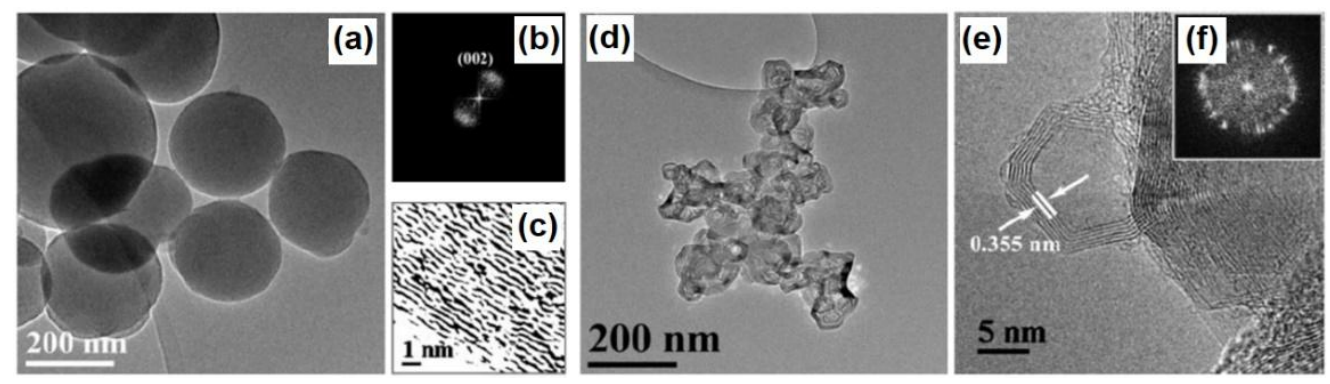

Figure 4 - Effect of high-energy relativistic electron beam irradiation (11.2 kW for 7 min) on the graphitic structure of thermal black. TEM image of CB before irradiation (a) with related fast Fourier transform (b), and detailed structure of the graphene layers at a particle surface (c). TEM images of $\mathrm{CB}$ after irradiation showing a resulting aggregate of 

nanocapsules (d), a nanocapsule close up (e) with related fast Fourier transform (f). Reproduced and adapted from reference [32].

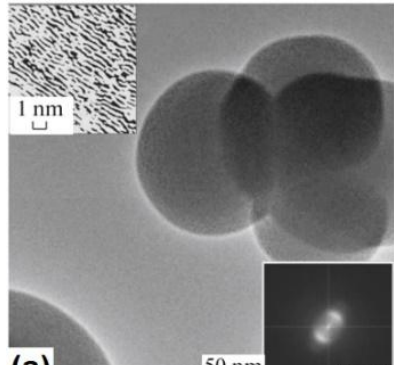

(a)

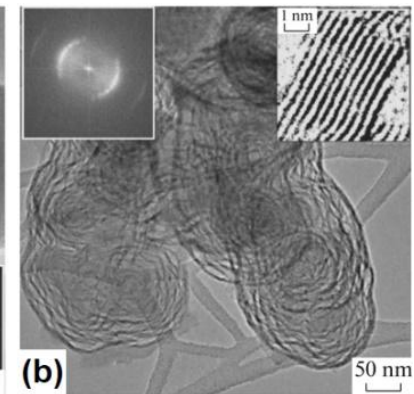

(b)

Figure 5 - Effect of high-energy laser irradiation $(1064 \mathrm{~nm}$; energy pulse $0.7 \mathrm{~J}$; length and frequency $8 \mathrm{~ns}$ and $10 \mathrm{~Hz}$, respectively) on the turbostratic graphitic structure of gas black (BLACK NRG® T900). HRTEM images of CB before (a) and after (b) laser irradiation in aqueous suspension. Insets show related fast Fourier transforms and details of the graphene layers. Reproduced and adapted from reference [33].

Thermal annealing is an excellent method to improve the lattice ordering in CB. Heating rate, duration, and temperature are key factors in determining the resulting graphene in-plane ordering and the reduced degree of stacking disorder (graphitization). Furnace heating is slow and effective in the scale of several minutes, whereas laser induced heating can achieve rates of millions of degrees per second. At this ultrafast heating rate, long range reconstructing of the stacked graphene domains is kinetically limited and only partial graphitization is observed. A striking example is given in Figure 6 where the stacking order of carbon black REGAL $® 250$ appears greatly enhanced after furnace heating at a rate of 25 ${ }^{\circ} \mathrm{C} /$ min holding at $2600{ }^{\circ} \mathrm{C}$ for one hour, Figure 6(a). However, when a similar temperature $\left(2850{ }^{\circ} \mathrm{C}\right)$ is achieved in a few nanoseconds using pulsed laser heating, lamellae are found to form concentric shells, Figure 6(b). At higher laser fluences, temperatures are so high that $\mathrm{C}_{2}$ sublimation occurs, making the nanoparticles burst and leaving behind entangled ribbon structures, Figures 6(c) and 6(d) [16]. Evidently laser-induced thermal treatment produces a diversity of carbon nanostructures. However, it is unclear how scalable the process is and whether the resulting carbon structures can be obtained in sufficiently large scale for applications. Nonetheless, future research work published on these materials properties in energy storage and conversion devices may push for further advances in the field provided properties exceed those of the starting CBs or of other graphene-based materials. 

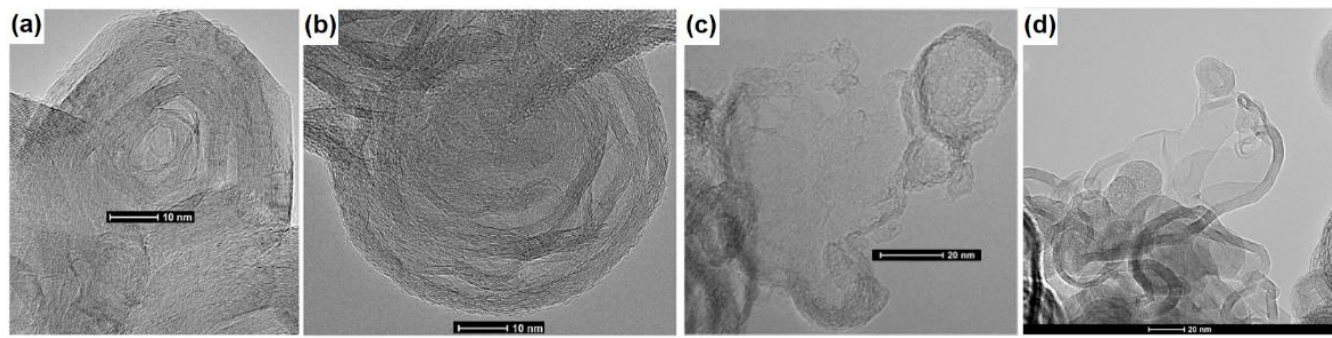

Figure 6 - Effect of heating method and temperature on the BSU integrity and structure of carbon black REGAL® 250. TEM images of CB after furnace heat treatment in argon 2600 ${ }^{\circ} \mathrm{C}$ (a), and $1064 \mathrm{~nm}$ laser heating at a fluence value of $50 \mathrm{~mJ} / \mathrm{cm}^{2}$ reaching a maximum temperature of about $2850{ }^{\circ} \mathrm{C}(\mathrm{b}), 300 \mathrm{~mJ} / \mathrm{cm}^{2}$ achieving the maximum temperature of $\mathrm{C}_{2}$ sublimation at $4180{ }^{\circ} \mathrm{C}(\mathrm{c})$, and $550 \mathrm{~mJ} / \mathrm{cm}^{2}$ causing significant vaporization of the sample (d). Reproduced and adapted from reference [16].

Microwave treatment can also be applied to alter and modify the structure of CB. In this case, a combined thermal (phonons generation), electron (electronic excitation), and ion (surface ionization) effect has been proposed to explain the structural changes observed upon microwaving [41]. Atom displacement, bond breaking, and crosslinking of activated surfaces were proposed to be the cause of major structural changes transforming CB agglomerates into clusters of hollow nano-balls, or higher aspect ratio nano-sticks, or even into entangled nano-wire like patterns depending on the amount of energy input (Figure 7). The round (balllike) and elongated (stick-like) shapes of the resulting products were dependent on different structural orientation in CB, "spheroidal" and "fibrous" orientations, respectively. These orientations were probably influenced by the dispersion of $\mathrm{CB}$ in toluene followed by hot plate heating at temperatures up to $500{ }^{\circ} \mathrm{C}$ [41]. $\mathrm{CB}$ can be converted to hollow carbon nanospheres through chemical methods. One recent example is the work of Wutthiprom et al. [42] applied to Li-ion batteries. In their work, $\mathrm{CB}$ was first oxidized in $\mathrm{HNO}_{3}$ at $100{ }^{\circ} \mathrm{C}$ for 96 hours, and then reduced with hydrazine in water at $100{ }^{\circ} \mathrm{C}$ for 36 hours. The resulting hollow carbon nano-spheres are shown in Figure 8. While Raman provided evidence for greater ordering of the hexagonal carbon lattice, fast Fourier-transform (FFT) applied to HRTEM characterization results supported the formation of local graphitic carbon domains [42].

The size and shape of graphene layers in CB have been determined from powder X-ray diffraction data. The best fit of the two-dimensional 10 reflections in the diffraction profile of CB was found by applying the Debye equation to a single simulated graphene sheet while 
varying its shape, size, and interatomic distances within the aromatic layer [43]. The results indicate that the ordered regions within the graphene layers are elliptical in shape and curved along the longer axis of the ellipse, matching to those experimentally observed using TEM (Figure 9). Individual GO-like nanosheets with characteristic blue photoluminescence can be peeled off from the surface outer layers of $\mathrm{CB}$ via oxidative treatment with $\mathrm{KMnO}_{4}$ in $\mathrm{H}_{2} \mathrm{SO}_{4}$ (Figure 10) [18], an easy and potentially scalable method to prepare GO-like products from inexpensive $\mathrm{CB}$ for energy applications. Ultrasound irradiation can also be used to produce carbon nanosheets from CB, this was achieved with carbon black Cabot N330 dispersed in water after prolonged (44 h) bath ultrasonication (90-W) [44]. Using an ultrasonic processor to input more power $(300-\mathrm{W})$ over a shorter time $(1 \mathrm{~h})$, localized short-range order of BSU was induced in low structure carbon black Cabot MOGUL® L, however this order was lost after prolonged sonication ( $3 \mathrm{~h}$ ) (Figure 11). Electron energy loss spectroscopy was used to quantify the ratio of $\mathrm{sp}^{2}: \mathrm{sp}^{3}$ carbon in the sample after ultrasonication, the ratio dropped from 77:23 for MOGUL® L to 55:45 for the ultrasonicated sample $(300-\mathrm{W} / 3 \mathrm{~h})$ with a corresponding loss of UV absorbance at $250 \mathrm{~nm}$ [45]. This conversion from $\mathrm{sp}^{2}$ to $\mathrm{sp}^{3}$ hybridization, due to BSU oxidation, is an important factor to consider when processing $\mathrm{CB}$ for specific applications as discussed in the later sections of this manuscript.

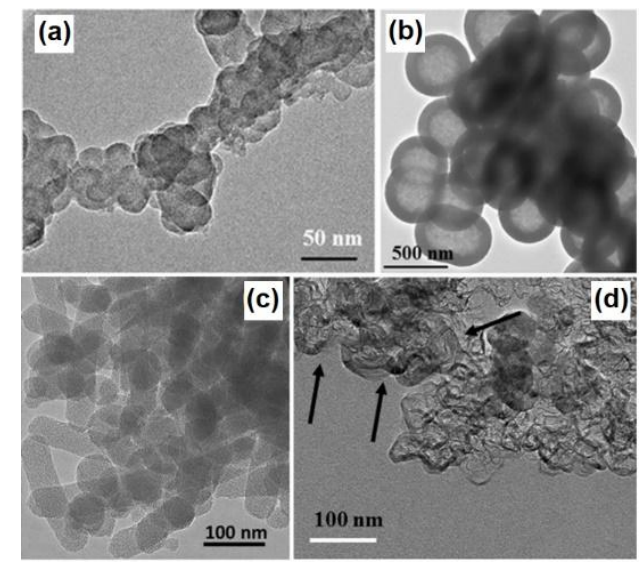

Figure 7 - Effect of microwave treatment on the structure of conductive carbon black ENSACO ${ }^{\circledR} 350 \mathrm{G}$ from Imerys Graphite \& Carbon. HRTEM images of an agglomerate of pristine CB (a), and of the modified carbon structures formed upon exposure to $900-\mathrm{W}$ microwave radiation for $15 \mathrm{~min}, 30 \mathrm{~min}$, and $45 \mathrm{~min}$ to make carbon nanoballs (b), nanosticks (c), and nano-wires like patterns with high graphitized regions as showed by the arrows (d), respectively. Reproduced and adapted from reference [41]. 

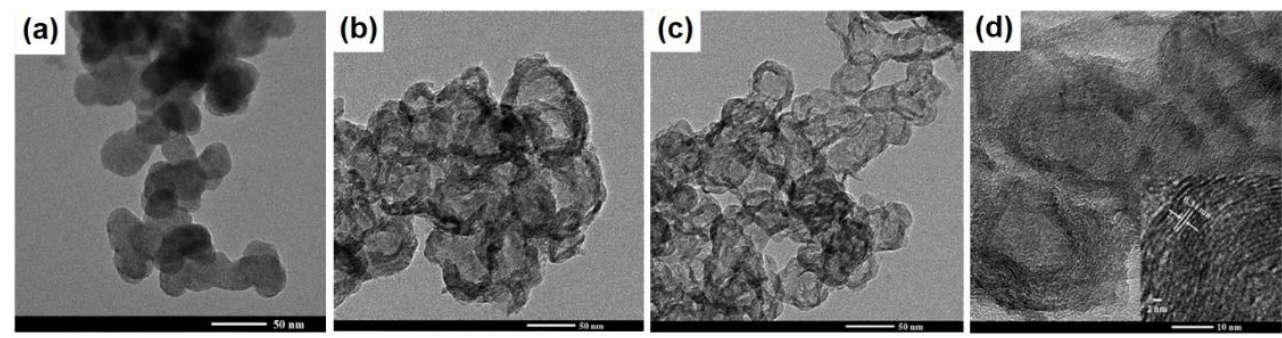

Figure 8 - Conductive carbon black Super $P ®$ from Imerys Graphite \& Carbon converted to hollow carbon nano-spheres using a chemical method. TEM images of a pristine aggregate (b), the hollow carbon structures produced after oxidation with $\mathrm{HNO}_{3}$ (b) converted to hollow carbon nano-sphere upon chemical reduction with hydrazine (c), and HRTEM of the localized graphitic domains present in the shells (d). Reproduced and adapted from reference [42].
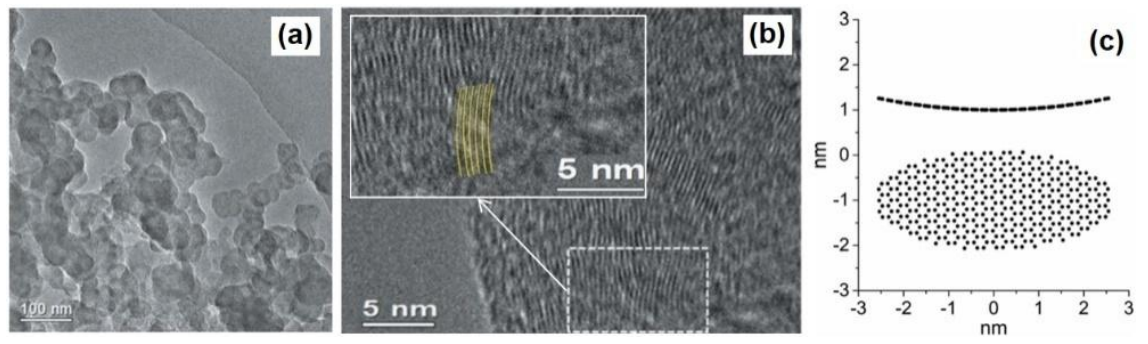

Figure 9 - Size and shape of ordered regions within the graphene layers of conductive carbon black Super P®. TEM images of aggregate of Super $\mathrm{P} \circledast$ nanoparticles (a), close ups of a primary particle with the yellow lines showing ordered layer profiles in the inset (b). The curvature of the layer profile agrees with that calculated using the Debye equation for elliptically shaped ordered regions (c). Reproduced and adapted from reference [43].
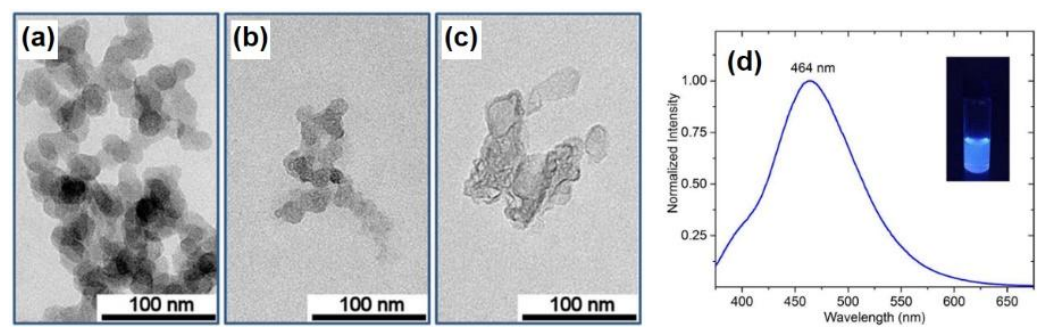

Figure 10 - Oxidative peeling off of graphene oxide-like nanosheets from CB produced using a laser-assisted combustion process $[18,46]$. TEM images of pristine CB aggregates (a), an aggregate after oxidative treatment in $\mathrm{KMnO}_{4} / \mathrm{H}_{2} \mathrm{SO}_{4}$ (b), resulting exfoliated GO-like nanosheets (c), and their photoluminescence spectrum recorded in aqueous solution at 350 
$\mathrm{nm}$ excitation (in the inset a photo of the same solution under a $365 \mathrm{~nm}$ UV lamp) (d). Reproduced and adapted from reference [18].
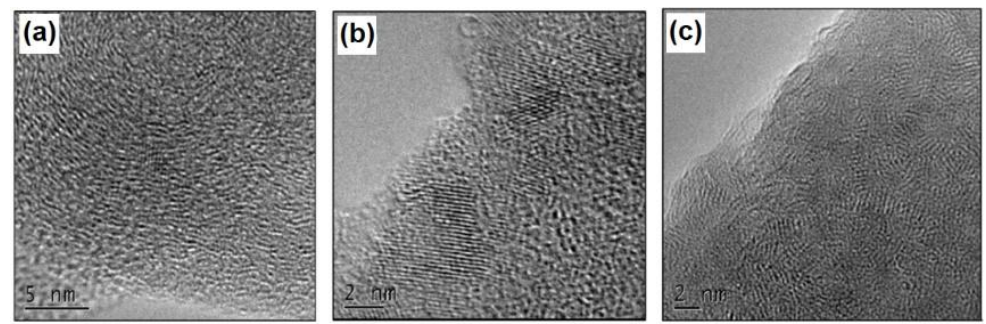

Figure 11 - Effect of ultrasonic treatment on the localized graphitic structure of carbon black Cabot MOGUL® L. HRTEM images of pristine CB (a), and after ultrasonication at 300-W for $1 \mathrm{~h}(\mathrm{~b})$ and $3 \mathrm{~h}$ (c). Reproduced and adapted from reference [45].

The internal structure of CB nanoparticles can be micro and/or mesoporous. Evidence for this is found in the comparison of the external surface area of the primary particles and the total or specific surface area that includes internal particle pores. The external surface areas of $\mathrm{CBs}$ are of major importance to the rubber and polymer composite industry, as these provide a measure of the accessible CB surface to rubber [47]. Both external and total surface areas can be calculated by the statistical film thickness method (STSA) and by the BrunauerEmmett-Teller (BET) method for the $\mathrm{N}_{2}$ adsorption isotherms at $77 \mathrm{~K}$, respectively. When measuring an adsorption isotherm, $\mathrm{N}_{2}$ strongly adsorbs on both the external and internal surface of the particles. The amount of gas uptake is plotted with respect to the relative pressure of $\mathrm{N}_{2}$ at which adsorption takes place. Characteristic adsorption isotherms are thus obtained and classified based on the types of or lack of pores [48, 49].

The BET method is based on the assumption of a statistical film formed on the surface of the material under investigation and it describes the monolayer-multilayer adsorption isotherm for nonporous sorbents. The linear form of the BET equation allows for estimating the volume of gas required to form a monolayer, and this value consequently leads to the surface area of the solid [48-50]. Even though the BET method was derived for a macroporous sorbent, it has been widely used for the investigation of microporous and mesoporous materials. In the presence of pores, the monolayer volume includes the volume required to fill small micropores, and the statistical film formed within larger micropores, mesopores and small macropores depending on the selected partial pressure range for linear fittings. This leads to difficulties in estimating the correct $\mathrm{N}_{2}$ monolayer capacity, and subsequently SSA 
for porous solids [51-53]. Consequently, results from the BET method for the same materials vary among different authors. However, the method has been widely accepted for comparing series of samples exhibiting similar adsorption properties, including microporous and mesoporous materials. The interpretation of the BET values may be better appreciated by different authors provided the relative pressure range is reported. For instance, Choma and Jaroniec located the monolayer range for the BET method to lie within 0.05 and 0.30 for nonporous carbons and for carbons having small macropores based on adsorption potential distributions [54]. The latter range has also been found valid to the study of mesoporous nanocomposites and carbon materials $[48,55]$. For most microporous carbons, the monolayer formation may take place within lower relative pressure ranges, and authors may find the set of recommendations published by Rouquerol, et al. helpful in locating the monolayer range [53].

The t-plot method, on the other hand, makes use of nonporous sorbents as reference for the analysis of other sorbents having similar chemical composition and surface properties. Consequently, the calculated surface areas from the t-plot are relative to the reference sorbent. The statistical film thickness is obtained by multiplication of the monolayer thickness of adsorbed gas by the surface coverage $(\theta)$ for reference solid [56]. The surface coverage is the ratio of the amount of gas adsorbed by the monolayer capacity (or monolayer volume) of the reference sorbent. A general equation for the reference solid can be obtained for the curve relating the statistical film thickness to the relative pressure. In case of $\mathrm{CB}$, the reference N762 material was used as reference for the equation for its low surface area and low structural level [47]. The gas uptake of a sample under investigation is then plotted against the calculated statistical film thickness for the sample using the equation for the reference solid. The statistical film thickness surface area (STSA) is thereby obtained by linear fitting of the obtained curve. For nonporous or macroporous sorbents, the t-plot (or STSA method for CB) yields the surface areas for the statistical film thickness of adsorbed $\mathrm{N}_{2}$ on the external particle surfaces, which is in the range between $0.35 \mathrm{~nm}$ and $0.50 \mathrm{~nm}$ for the reference carbon surface [56]. When micropores are present in the investigated sample, the calculated STSA surface area within this range is that of the accessible micropores [56]. The external surface area in the latter case is obtained by subtracting the STSA values from the BET surface areas [47].

Moreover, the use of a suitable reference carbon material offers a very powerful method for modeling adsorption isotherms, allowing for the accurate investigation of properties such as 
surface adsorption potential changes from surface heterogeneities, e.g. functional groups and pores [51, 52, 57]. Of interest to carbon materials, recent adsorption studies demonstrated that given the adsorption characteristics of non-graphitized BP280, this material is a suitable reference for adsorption studies of other carbon blacks and of porous carbons. In this direction, comparative plot analysis of the $\mathrm{N}_{2}$ adsorption isotherms for BP280, including the model independent $\alpha$-plot $[48,49]$, further led to more accurate methods to calculate adsorption potential and pore size distributions for microporous and mesoporous carbons [51, $52,55,57]$. These results were also extended for other gas probes such as Ar at $87 \mathrm{~K}$ for the same BP280 as the reference material [58].

Given the model restrictions and the need for careful analysis of the fitting ranges used in each method, the external surface areas from STSA equation and the BET method are the industry standard for calculating primary particle size (PPS) [47]. For calculating the PPS, the external surface area value is combined with an assumed spherical geometry of the individual particles forming linear aggregates. The PPS is the average thickness of the aggregate chain and its value is inversely proportional to the external surface area of the material [47]. Besides gas adsorption methods, TEM provides an additional method to directly image and estimate the geometry and PPS of linear CB aggregates, and consequently, to estimate the geometrical surface areas of these materials.

As a rule of thumb, $\mathrm{CB}$ is microporous when the specific surface area from $\mathrm{N}_{2}$ adsorption is significantly larger than that from the geometrical surface area from STSA calculations or from TEM. This has been reported for carbon black CARBOLAC 1 where the BET area is 3.7 times larger than the TEM area, whereas the two areas are practically the same for carbon black MOGUL® A [13]. Consequently, CARBOLAC 1 has micropores, whereas MOGUL® particles lack internal particle porosity. Carbon black MONARCH® 1300 has been reported to have a BET specific surface area of $554 \mathrm{~m}^{2} / \mathrm{g}$, while CB N762 of just $32 \mathrm{~m}^{2} / \mathrm{g}$, also providing evidence for the significant microporosity of the former with a micropore volume of $0.12 \mathrm{~cm}^{3} / \mathrm{g}$ [59]. The internal particle microporosity can be correlated to the inefficient random packing of large BSUs, leading to a higher free volume space between these, an hypothesis supported by molecular simulations [17]. Another factor affecting porosity is the presence of in-layer nonhexagonal defects that make the graphene sheets ripple and interfere with their stacking inducing nanoporosity [23, 60], a phenomenon observed in ultramicroporous carbon materials that might play a role in some highly porous CBs. Nonetheless, significantly larger voids can also be present. An outstanding example is the 
hierarchical microporous-mesoporous structure of highly electrically conductive Ketjenblack ${ }^{\circledR}$ (AkzoNobel) with BET surface areas reaching $1400 \mathrm{~m}^{2} / \mathrm{g}$. Figure 12 shows the mesopore structure (hollow cores) of Ketjenblack® $\left(1445 \mathrm{~m}^{2} / \mathrm{g}, 2.07 \mathrm{~cm}^{3} / \mathrm{g}\right)$ compared to acetylene black (Sterm Chemicals, $66 \mathrm{~m}^{2} / \mathrm{g}, 0.14 \mathrm{~cm}^{3} / \mathrm{g}$ ) [61], including a magnification of a typical mesopore [62]. The presence of mesopores increases the total surface area and accessibility of $\mathrm{N}_{2}$ to micropores in the carbon shells. Ketjenblack® can be further activated in air at $600{ }^{\circ} \mathrm{C}$ to further increase the surface area and the pore volume $\left(1600 \mathrm{~m}^{2} / \mathrm{g}, 4.15\right.$ $\mathrm{cm}^{3} / \mathrm{g}$ ), but also oxygen content from $4.2 \%$ to $6.7 \%$ for the pristine and 10 -min air-treated $\mathrm{CB}$, respectively. Further oxidation, however, appeared to compromise the mesoporous structure with the surface area dropping to $1030 \mathrm{~m}^{2} / \mathrm{g}$ after $30-\mathrm{min}$ of treatment [63]. Ketjenblack ${ }^{\circledR}$ can also be thermally annealed to increase electrical conductivity. The annealing leads to longer stacked graphene domains with an interspace distance of about 0.34 $\mathrm{nm}$, distinctive of graphitic carbons, Figure 12(d) [64]. Interestingly, the structure of graphitized Ketjenblack ${ }^{\circledR}$ is similar to that of Color Black FW200 heat-treated at $2600{ }^{\circ} \mathrm{C}$, Figure 2(h), and of thermal black after relativistic electron beam irradiation, Figure 4(e). The important difference that a much lower temperature $\left(1300^{\circ} \mathrm{C}\right)$ is required to obtain similar turbostratic stacked graphene shells in Ketjenblack® than in Color Black. In both examples, the growth of the BSUs into longer stacked turbostratic graphene domains resulted in growth of the conjugated $\mathrm{sp}^{2}$ network. Consequently, the greater electronic delocalization within each graphene plane provided the mechanism for the enhanced electrical conductivity in annealed materials [65].
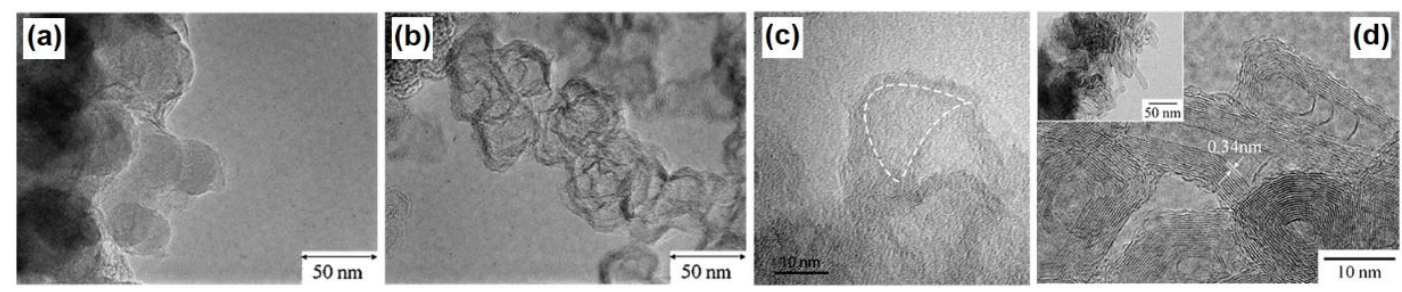

Figure 12 - Mesoporous structure of carbon black Ketjenblack®. TEM images of acetylene black showing solid primary particles (a), and of Ketjenblack® EC-600JD with its characteristic hollow structure (b) and a highlight of a mesopore of Ketjenblack ${ }^{\circ}$ EC-300J (c). Graphitized structures obtained from the thermal annealing of Ketjenblack® EC-600JD in argon at $1300{ }^{\circ} \mathrm{C}$ for $12 \mathrm{~h}$ are shown in the TEM image (d), where the inset is a low magnification image of the same sample. Reproduced and adapted from references [61, 62, 64]. 


\subsection{Formation of particles and aggregates}

The partial combustion of hydrocarbon feeds is a remarkably complex process not entirely understood at present. Reaction kinetics, heat and mass transfers are intertwined to deliver rapid changes of gas composition, nuclei concentration, particle growth, and evolving morphology of resulting aggregates - all factors strongly dependent on composition of the feed, furnace design and performance, and operational conditions. It is thus no surprise that attempts to shed light in such a complex process look at simplified systems to extract intelligible information. Ono et al. [66-69] studied the pyrolysis of nitrogen-diluted benzeneacetylene mixtures to understand the dynamics of $\mathrm{CB}$ particle formation and aggregation. In general, there is agreement that during pyrolysis polycyclic aromatic hydrocarbons (PAH) form and grow into heavier PAH particles collectively termed as soot. The collision of soot produces larger spherical particles that then sinter into the final $\mathrm{CB}$ aggregates. By adding acetylene $(0.5 \%)$ to a benzene $(1.0 \%)$ feed, it has been experimentally documented that more branched aggregates form at high furnace temperature, whereas the aggregates are more spherical at lower temperature since the presence of small PAH enhances surface growth when benzene or acetylene concentrations are high (5\%) [66]. Figure 13 shows that adding acetylene to a benzene feed increases the nucleation rate, i.e., nuclei form earlier and faster in the process, exactly when a drop of acetylene concentration is also observed. Phenanthrene and pyrene (3- and 4-membered aromatics, respectively) form in parallel to increase nucleation, supporting that the acetylene-induced hydrogen-abstraction/carbon-addition (HACA) mechanism, responsible for the formation of PAH, is also linked to the increased branching of the aggregates [68]. This example shows how CB particle growth and aggregation are intimately related, although such evidence has been obtained under simplified process conditions different from those of the more complex industrial production processes. Sintering or fusing behavior of primary particles leading to aggregates has been linked to the pyrolysis temperature, that also affects particle growth, nucleation rate, and PAH formation. In particular, the pyrolysis of ethylene (1\% in nitrogen) produces aggregates with better refined branching and smaller particles with increasing temperature (Figure 14) [70]. A comparison of 100-nm-sized aggregates formed at about 1300, 1400, and $1500{ }^{\circ} \mathrm{C}$ shows that at higher temperatures, clusters are more complex in shape, as evidenced by 180nm-sized aggregates. Furthermore, at lower temperatures smaller aggregates consist of larger fused particles with little or no branching, see Figure 14(a). A similar temperature effect was 
observed in another study where an idealized benzene feed (1.2\% in nitrogen) was used, from which smaller primary particle and more complex shape agglomerates formed at high furnace temperature and short residence time. It was implied that high molecular weight PAH formed at higher temperature since they are the species responsible for nucleation, whereas low molecular weight PAH are involved in particles growth [71]. The particles subsequently fuse with each other to form a "welded" aggregate, as early studies of commercial furnace carbon blacks have highlighted the continuity of stacked graphene domains across different primary particles forming continuous clusters [72]. Aggregates can further fuse into larger globular clusters using microwaves [41], or by coating with graphitic material deposited on preformed CB aggregates via pyrolysis of a light carbon source such as propylene [73], as shown in Figure 15. Because of the complexity of the different structures formed, the characterization of $\mathrm{CB}$ particle size and shape distribution is a non-trivial task, and reproducible interlaboratory tests are difficult to develop [74].
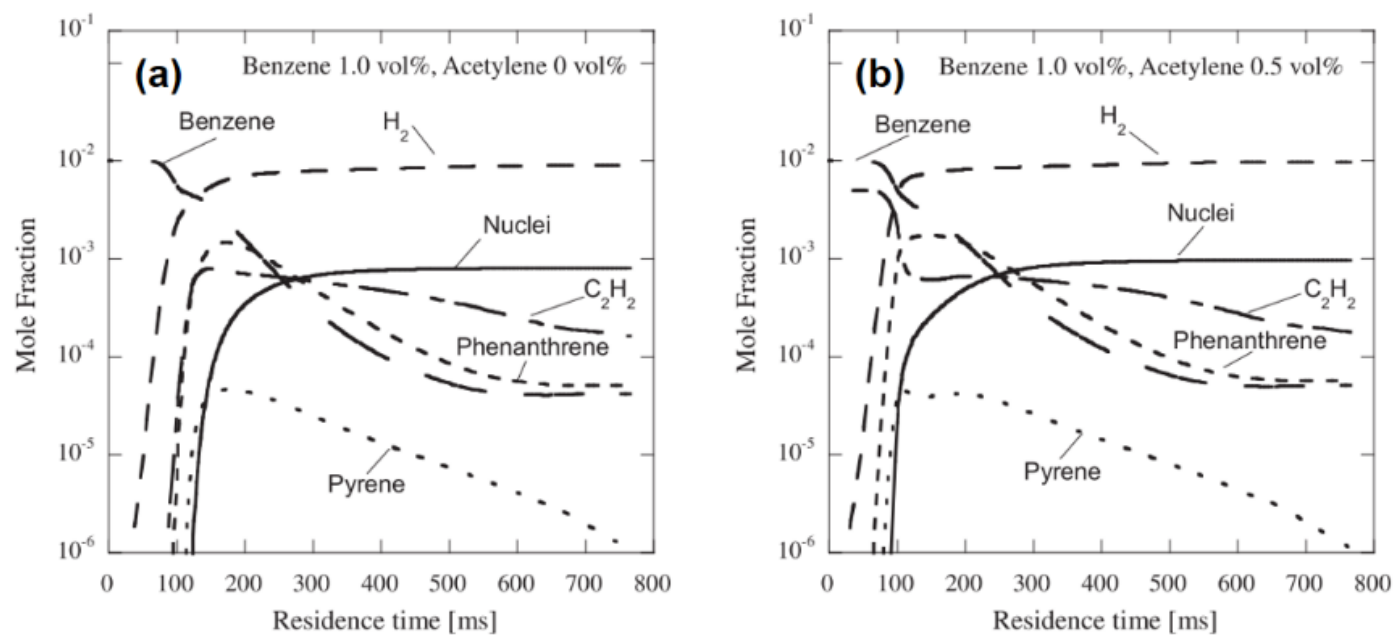

Figure 13 - Composition-time profiles of the main species involved in the formation of $\mathrm{CB}$ from benzene-acetylene mixtures. Benzene, acetylene, hydrogen, phenanthrene, pyrene, and nuclei mole fractions change during pyrolysis of $1 \%$ benzene in nitrogen (a), and the same with the addition of $0.5 \%$ acetylene (b). Reproduced and adapted from reference [68]. 

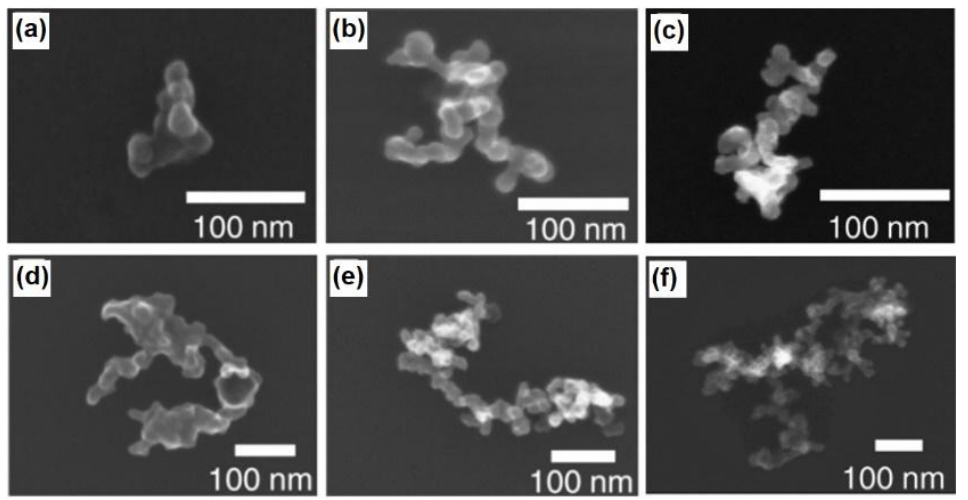

Figure 14 - CB aggregates of different sizes produced from the pyrolysis of ethylene at different temperatures, an informative yet idealized case compared to the carbon feeds used in industrial processes. SEM images of 100-nm-sized (a, b, c) and 180-nm-sized (d, e, f) aggregates produced at about $1300{ }^{\circ} \mathrm{C}(\mathrm{a}, \mathrm{d}), 1400{ }^{\circ} \mathrm{C}(\mathrm{b}, \mathrm{e})$, and $1500{ }^{\circ} \mathrm{C}(\mathrm{c}, \mathrm{f})$. Reproduced and adapted from reference [70].

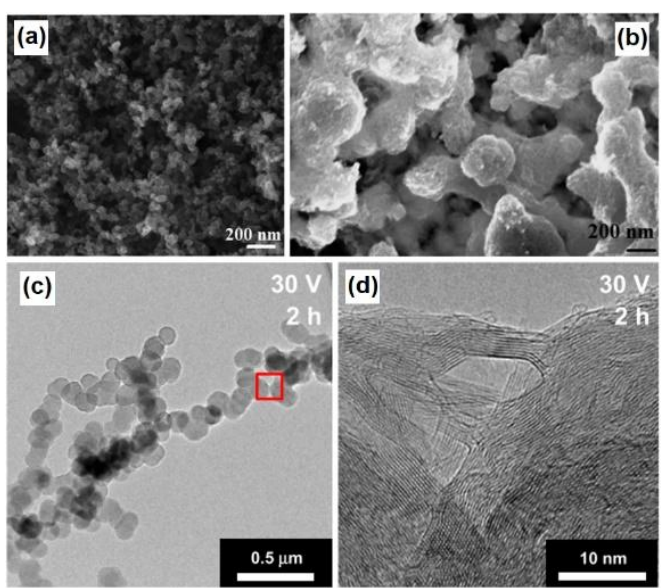

Figure 15 - Modification of carbon particle agglomerates. SEM images of as received ENSACO ${ }^{\circledR} 350 \mathrm{G}$ (a), and after heat treatment followed by microwave irradiation at $900-\mathrm{W}$ for $15 \mathrm{~min}$ (b). TEM images of multi-layer graphene-coated carbon particles produced from the pyrolysis of propylene (c, d). A constant voltage was used during pyrolysis to collect chain-like agglomerates that after collection were again exposed to the pyrolysis environment to produce graphitic layer preformed aggregates. The content in the red square (c) is magnified in image (d). Reproduced and adapted from references [41, 73].

\subsection{Alternative production methods}

Thermal plasma, liquid phase plasma, microwave and radiofrequency-generated plasma, arcdischarge non-thermal plasma, chemical looping pyrolysis, thermal conversion of biomass 
pyrolysis oil, and solar thermal conversion of hydrocarbon feeds have been all proposed and studied as alternatives to commercial $\mathrm{CB}$ production processes. At present, none of these alternatives have been deployed at a production scale as large as the commercial processes. Thermal plasma is considered a major energy efficient technology for tunable high temperature heat sources, especially when paired with renewable energy sources substituting conventional furnaces that operate on thermoelectric energy sources. $\mathrm{CB}$ has been produced from the thermal plasma of methane and polymers, and long studied and modeled pilot processes are now becoming commercially available [75-81]. Converting carbon-based feeds without combustion for energy input has the benefit to drastically reduce $\mathrm{CO}_{2}$ emissions. Monolith Materials Inc. has licensed the technology, developed and built a demonstration CB production plant using a thermal plasma process that emits $70 \%$ less $\mathrm{CO}_{2}$ and about a hundred times less nitrogen (NOx) and sulfur (SOx) oxides compared to the furnace process [76]. Liquid phase plasma has also been used to prepare CB. Two tungsten electrodes immersed in benzene were utilized to generate a plasma flux by pulsing high voltage to produce $\mathrm{CB}$ with an ordered carbon content greater than that of the highly conductive Ketjenblack® [82]. This $\mathrm{CB}$ was then tested in its pristine and $\mathrm{KOH}$-activated forms in supercapacitors, outperforming commercial $\mathrm{CB}$. The plasma and $\mathrm{KOH}$-activated $\mathrm{CB}$ achieved a gravimetric capacitance of $116 \mathrm{~F} / \mathrm{g}$ against $47 \mathrm{~F} / \mathrm{g}$ for activated commercial $\mathrm{CB}$ at a current density of $250 \mathrm{~mA} / \mathrm{g}$. These results were obtained for symmetrical supercapacitor coin cells using $1 \mathrm{M} \mathrm{LiPF}_{6}$ in ethylene carbonate/dimethyl carbonate (EC/DMC, 3:7 by volume) as the electrolyte solution [83]. In a different study, low temperature plasma was applied to convert methane into $\mathrm{CB}, \mathrm{C} 2$ products, and $\mathrm{H}_{2}$ [81]. Both microwave- and radiofrequency $(\mathrm{RF})$-generated plasma were used in the absence and presence of a bimetallic catalyst, $\mathrm{Pd}-\mathrm{NiO} / \gamma-\mathrm{Al}_{2} \mathrm{O}_{3}$. The catalyst was found to increase the overall conversion efficiency of the process from $60 \%$ to $70 \%$ using $800-\mathrm{W}$ RF plasma; the selectivity for CB also increased from about $20 \%$ to $30 \%$. The resulting plasma black had a BET surface area slightly below $45 \mathrm{~m}^{2} / \mathrm{g}$, which increased to approximately $68 \mathrm{~m}^{2} / \mathrm{g}$ at an applied power of $1000 \mathrm{~W}$.

Low-current/high-voltage arc plasma discharge was applied to crack propane into $\mathrm{CB}$ and $\mathrm{H}_{2}$ [84]. A small current plasma arc discharge was produced by applying a high voltage in argon (2 $\mathrm{kV}, 0-1 \mathrm{~A})$; next, propane was fed in the plasma to produce $\mathrm{CB}$. Under optimized conditions ( $\mathrm{Ar} 80 \mathrm{~L} / \mathrm{min}, \mathrm{C}_{3} \mathrm{H}_{8} 60 \mathrm{~mL} / \mathrm{min}, 0.9 \mathrm{~A}$ ), spherical carbon black particles with 50 $\mathrm{nm}$ average diameter and $96.7 \mathrm{~m}^{2} / \mathrm{g}$ BET surface area were obtained [84]. From TEM 
characterization, it was found that the resulting $\mathrm{CB}$ contained both acetylene-type and furnace-type nanoparticles (Figure 17). The acetylene-type $\mathrm{CB}$ had a well-arranged and extended domains resembling the graphitic structure in the shell structure formed under HACA conditions, typical of acetylene-rich feeds, Figure 17(b). The furnace-type, on the other hand, shows a turbostratic structure with disordered graphene layers staking due to random layer rotation and translation causing alignment mismatch, shown in Figure 17(c). Coupling arc discharge non-thermal plasma with thermal pyrolysis in tandem allowed to improve the content of ordered graphene units in the resulting CB. Consequently, the electrical performance with a drop of resistivity from 1000 to $800 \Omega \cdot \mathrm{m}$ measured for CBpolymer binder composites (Figure 18) [85].
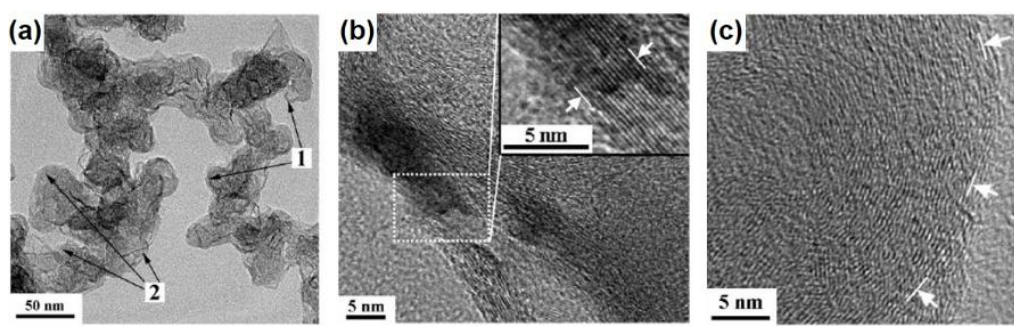

Figure 16 - CB produced by plasma arc discharge. TEM image of arc plasma CB aggregates merging two typologies of $\mathrm{CB}$, as shown by the arrows, 1) acetylene-type black and 2) furnace-type black with their HRTEM images provided in (b) and (c), respectively. Reproduced and adapted from reference [84].
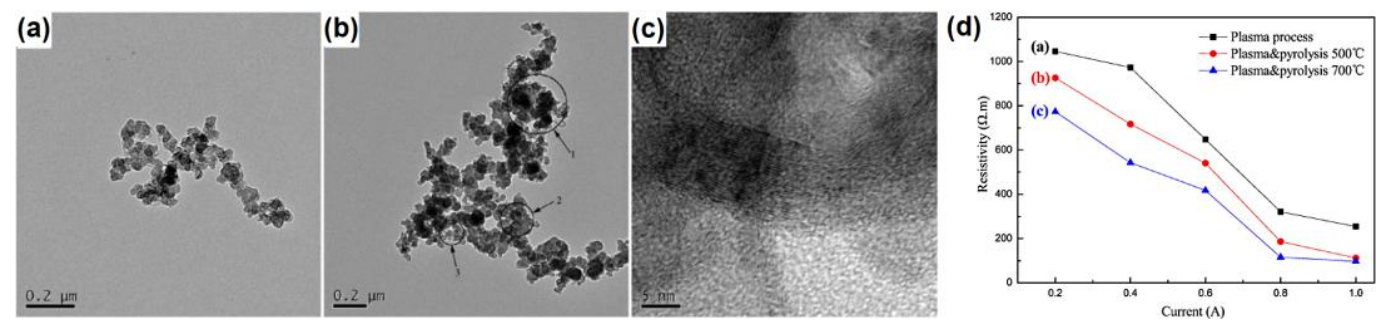

Figure 17 - CB produced by plasma arc discharge in tandem with thermal pyrolysis. TEM images of arc plasma CB prepared under plasma process only (a), and in tandem with thermal pyrolysis (b). HRTEM image showing the extended domains resembling graphitic carbon structure of arc plasma-thermal annealed CB (c). Comparison of electrical performance of composites made of $\mathrm{CB}(60 \mathrm{wt} \%)$ and polyvinylidene fluoride binder (40 wt.\%) (d). Reproduced and adapted from reference [85]. 
Chemical looping pyrolysis has also been investigated in order to decrease the energy demand and environmental footprint of CB production [86]. The principle of operation is the use of an oxygen carrier (OC) particulate material to transfer lattice oxygen to the pyrolysis reactor from an air reactor utilized to regenerate the OC. The role of lattice oxygen (i.e., oxygen transported in the material lattice of the OC) is to extract hydrogen from the carbon feed, thus promoting the generation of $\mathrm{CB}$. The resulting chemically reduced $\mathrm{OC}$ is then reoxidized in the air reactor to be reused in the next pyrolysis cycle. Using an OC allows to reduce process temperature, energy consumption, and emissions. Tested OCs were hematite $\left(88 \% \mathrm{Fe}_{2} \mathrm{O}_{3}\right.$ with alumina and silica as the next most abundant components) and gypsum $\left(93 \% \mathrm{CaSO}_{4}\right.$ with a balance of magnesia). Hematite produced $40 \mathrm{~nm}$ and $20 \mathrm{~nm} \mathrm{CB}$ particles at 800 and $900{ }^{\circ} \mathrm{C}$, respectively; gypsum produce particles with a wider size distribution from $10 \mathrm{~nm}$ to $100 \mathrm{~nm}$. CB yields increased when smaller diameter OC particulate was used; however, $0.1 \mathrm{~mm}$ OC were found to be incorporated as impurity in the resulting CB products [86]. Alternatively, bio-based "green carbon black" has been recently reported. This approach converted pyrolysis oil (PO) from pine and spruce stem wood into CB [87], in a thorough and detailed work, where process conditions were examined to produce green $\mathrm{CB}$ with structural features similar to commercial $\mathrm{CB}$ materials. For instance, at $1300{ }^{\circ} \mathrm{C}, 10.6 \%$ of fed PO was converted to $\mathrm{CB}$ with structural characteristics similar to medium disperse $\mathrm{CB}$ commercial grades. Graphitization was enhanced at higher temperature, $1700{ }^{\circ} \mathrm{C}$. However, the high oxygen content of PO was a source of intensified oxidation at higher process temperatures, causing selective shrinking of nodules and consumption of small agglomerates, thus impacting the green CB agglomerate size distribution and resulting performance [87]. Finally, a different work reported the use of solar energy to provide the thermal energy required to split methane into carbon $(\mathrm{CB})$ and $\mathrm{H}_{2}$. These findings showed that conversion yields to $\mathrm{CB}$ of up to $63 \%$ could be achieved [88]. Furthermore, a process analysis for a 55 MW solar power plant was proposed to competitively produce $1.7 \mathrm{t} / \mathrm{h}$ and $5 \mathrm{t} / \mathrm{h}$ of $\mathrm{H}_{2}$ and $\mathrm{CB}$, respectively [89]. Hence, the aforementioned recent findings demonstrate a promising future for the $\mathrm{CB}$ market provided high process yields, green energy use, low $\mathrm{CO}_{2}$ emission are achieved, and where CB materials with tunable properties are obtained in large scale using abundant renewable resources, and generating valuable byproducts, namely $\mathrm{H}_{2}$.

\subsection{Carbon black electrical properties}

Among the different properties of $\mathrm{CB}$, one of the most relevant for energy application is 
electrical conductivity since charge transport is often the most common phenomenon involved in low carbon energy storage, generation, and conversion. As discussed in the previous sections, $\mathrm{CB}$ materials are aggregates of carbon nanoparticles each made of sub to a few nanometer scale stacked graphene BSU grain domains. This results in a mostly fragmented electronic conduction pathway. Electrical conductivity $(\sigma)$ in $\mathrm{CB}$ is the result of a complex combination of intra- and inter-particle charge transport. Consequently, $\sigma$ is affected by a series of conduction resistances at BSU/BSU and particle/particle contacts. These resistances dominate over the intrinsic conductivity of single BSUs and primary particles. Research effort has focused on understanding electrical conduction at both intraparticle (localized) and interparticle (extended) level. Microwave techniques have been applied to provide bulk measurements of intraparticle charge conductivity and mobility. This is possible since microwave cavities eliminate the need for electrode contacts and the frequency used avoids conduction across grain boundaries and particle-particle contacts [90]. Studies of BLACK PEARLS® 2000 reported values of intraparticle electron conductivity, mobility, and carrier density of $2.4 \mathrm{~S} / \mathrm{cm}, 5.4 \mathrm{~cm}^{2} /(\mathrm{Vs})$, and $1.5 \times 10^{18} / \mathrm{g}$, respectively [90]. In comparison, the carrier density in polycrystalline graphite is $10 \times 10^{18} / \mathrm{g}$. Furthermore, holes are the primary charge carriers in the graphene domains of CB [90].

The interparticle electrical contact determines the electrical properties of compacted CB. It is thus no surprise that the electrical conductivity depends on the particle size and separation distance, the nature and amount of impurity and/or functionalization present on the surface. Thermal treatment to remove oxygen and sulfur functional groups is a common procedure to increase carbon black conductivity, complementary to thermal annealing used instead to increase graphitization and consequently intraparticle and intra-aggregate electrical conduction. The interparticle conduction mechanism is dominated by electron tunneling governing the transfer of electrons across the particle-particle contacts. The contacts can be altered under mechanical compression, affecting particle packing and as a result the number of contacts between particles. In a study, the electrical conductivity of six CB grades has been characterized under applied pressure, relevant results are presented in Figure 16 [91]. Clearly, the values of $\sigma$ are strongly dependent of the bulk density of the compressed CB powder, with the bulk density dependent on the change of volume it occupies under the applied pressure. The occupied volume is a result of the particle size and intrinsic porosity, the aggregate structure and packing ability, among other factors. Low structure and coarse Sterling ${ }^{\circledR} \mathrm{V}$ achieved high bulk densities and corresponding high electrical conductivities, 
whereas highly structured, high surface area BLACK PEARLS® 2000 could only be packed to give low bulk densities resulting in significantly lower values of $\sigma$ [91]. This example shows how the conduction across aggregates is strongly dependent on the number of contacts and conduction pathways present in the bulk. At lower density with more voids present, there are less available conduction pathways and the resulting electrical conductivity is lower. The likelihood of contact between $\mathrm{CB}$ particles increases with increasing bulk density, i.e., decreasing void volume. A similar dependence of $\sigma$ vs. bulk density has been reported for Ketjenblack® EC-600JD, with compacted powder $\sigma$ values over ten times larger upon doubling the bulk density, going from 0.05 to almost $0.6 \mathrm{~S} / \mathrm{cm}$ [92]. It is relevant to compare the intraparticle (grain domain-confined) and interparticle (bulk-extended) electrical conductivity of BLACK PEARLS ${ }^{\circledR} 2000$, the two are $2.4 \mathrm{~S} / \mathrm{cm}$ and around $0.2 \mathrm{~S} / \mathrm{cm}$ (Figure 16), respectively, highlighting the detrimental effect of contact resistances on the macroscopic electrical performance of CB.

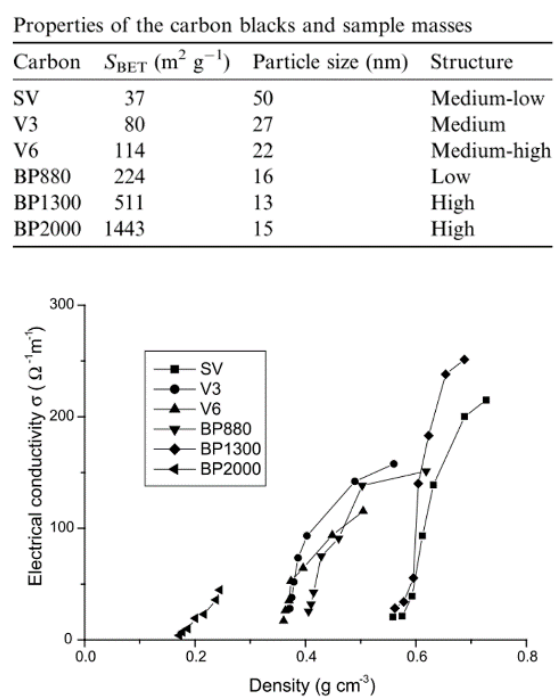

Figure 18 - Effect of bulk density of compacted CB powders on their electrical conductivity. In the table, a list of the properties of six Cabot CBs: Sterling® V (SV), VULCAN® 3 (V3) and 6 (V6), BLACK PEARLS 880 (BP880), 1300 (BP1300), and 2000 (BP2000). In the plot, the electrical conductivity of the six CBs plotted against their bulk density. Reproduced and adapted from reference [91].

In practical applications CB is rarely used alone, being instead combined with other components to form composites. Common binding components in composites are organic polymers having electrical insulating properties. A comprehensive description of electrical phenomena in CB-polymer composites has been provided by Balberg [93]. Different types of 
CB particles yield conducting networks with different interparticle distances affecting the resulting electrical behavior. The critical amount of $\mathrm{CB}$ required to achieve charge percolation (i.e., to switch the composite from electrically insulating to conducting) and how rapidly conductivity increases with increasing added amount of CB are factors influenced by the aggregates structure. Low structure and high structure aggregates of spheroidal and elongated shape, respectively, do contact differently in the polymer matrix. In particular, high structure aggregates can entangle to give a narrow distribution of interparticle distances, thus creating a network of concentrated CB clusters surrounded by volume parts of polymer with no significant CB clusters in them [93]. It is then possible that less CB is needed to achieve percolation. Above the percolation threshold, when the concentration of CB is high enough, other studies propose that conduction proceeds via geometrical contact (such as that between neighboring metal granules) [94]. A polymer commonly used in energy application with CB is poly(vinylidene fluoride), PVDF. CB-PVDF composites are characterized by a positive temperature coefficient (PTC) effect where upon temperature increase the electrical conductivity decreases due to volume expansion. The latter increases the space separation between adjacent particles, to increase further due to agglomeration of $\mathrm{CB}$ particles induced by diffusion from amorphous polymer region to melted crystalline regions [95]. PVDF is used as a binder to hold $\mathrm{CB}$ aggregates in place with other added components depending on the type of application. The spreadability of CB is also another important factor determining the electrical conductivity of the resulting composite. CB nanoparticles spontaneously form aggregates with characteristic highly compressible microstructures. The ability to flow and spread under mechanical stress (e.g., compression, deformation) allow for the formation of squishable electrically conductive networks especially important in batteries and supercapacitors. Also binder-free CB compacts have demonstrated viscoelastic behavior, with elasticity being the main character under dynamic compression [96].

\section{Chemical functionalization}

In this section, we report key developments and findings on the chemical modification of CB. Key developments in this field are discussed in parallel with details of the type and grade of CB when available. The aim is to find useful correlations between CB structure, modification, and application. We hope to stimulate interest in the chemistry of CB and help identify opportunities for its applications in energy and environmental technologies. 
CB materials have intrinsic surface functionalities, mainly with carbon forming bonds to oxygen and hydrogen. As discussed in the introductory sections, the content and nature of groups vary according to the preparation methods. Among the different types of $\mathrm{CB}$, channel black has the highest amounts of oxygen, typically varying between 3-8 wt.\% [13, 97-106]; most CB have significantly lower surface functional groups, thus requiring harsher chemical conditions to form new $\mathrm{C}-\mathrm{C}$ bonds or oxidation to allow for the grafting of species bearing different functional groups. The latter reactions are of great importance, as channel black has no longer been produced in the United States and in most other countries since the 1960s. The channel black process suffered from low productivity and high environment impact compared to the cleaner processes of furnace and thermal blacks. A more efficient and environmentally friendly process, called gas black, is currently in use for specialty products. These share similarities in the production of channel black, thus keeping the literature of channel black relevant. Nonetheless, the gas black process relies on using coal tar distillates as feedstock instead of natural gas used for channel black [107].

Similar to most carbon allotropes, CB tends to be non-dispersible in most organic solvents, and aqueous media due to the strong van der Waals forces between its primary aggregates forming large entangled secondary aggregates. The poor dispersibility is also due to the average particle size of a given CB type, with the primary particle size varying from less than $10 \mathrm{~nm}$ to few hundreds of nanometers. Hence, dealing with the surface chemistry of $\mathrm{CB}$ particles has attracted considerable attention to increase its colloidal stability leading to better handling, processability, and broader spectrum of applications.

The chemical modification of CB is carried out via well-known methods mostly shared with those of activated carbons. These methods have subsequently been applied and modified for single and multiwall carbon nanotubes (CNT) [108], graphene [109] or even graphite in some occasions [110-112] for surface functionalization and nanocomposite manufacturing. In addition, the covalent functionalization of $\mathrm{CB}$ can be more generally divided into two categories: one comprising the introduction of macromolecules such as polymers, and the other comprising the surface functionalization using small molecules. In both cases, the covalent surface modification of CB proceeds either with preliminary oxidation of the carbon surfaces, thus adding alcohol, lactone, ether, ester, carboxylic acid functionalities, etc. [113] or by direct formation of carbon-carbon bonds through aryl diazonium salts, Diels-Alder, and radical reactions. Due to the abundance of literature sources detailing the chemistry of carbon surface, and its scientific and technological importance, herein, a series of the most important 
developments covering the covalent functionalization of CB is reviewed. Firstly, the surface oxidation methods and subsequent modification with alkyl or polymers are discussed, and secondly the direct C-C formation methods for CB are reviewed.

\subsection{Oxidation}

The surface properties of carbon black cannot always be tailored by the various existing manufacturing processes, which in turn limits the use of $\mathrm{CB}$ in some applications. Motivated by the need to improve dispersibility or hydrophilicity of $\mathrm{CB}$ particles in aqueous medium and other polar solvents, the oxidation of $\mathrm{CB}$ has been widely explored over the years. The use of surface oxidation methods aims at overcoming agglomerates formation found for pristine $\mathrm{CB}$ particles, as this is a critical issue for many industrial applications.

The key advantage of oxidizing CB is the introduction of reactive surface groups, which allow for further modification of particles aimed at specific applications. Up to now, three general approaches have been applied for CB oxidation, namely wet oxidation, gas oxidation and plasma oxidation. Figure 19 illustrates different examples of graphene edge oxygen functionalities in the BSU structure as proposed by Fanning et al. [113] and also found for soot [114]. From a chemical perspective, soot and CB share similar reactivity while still being two different materials [115]; hence, both share similar variety of oxygen functional groups formed during the oxidation process.

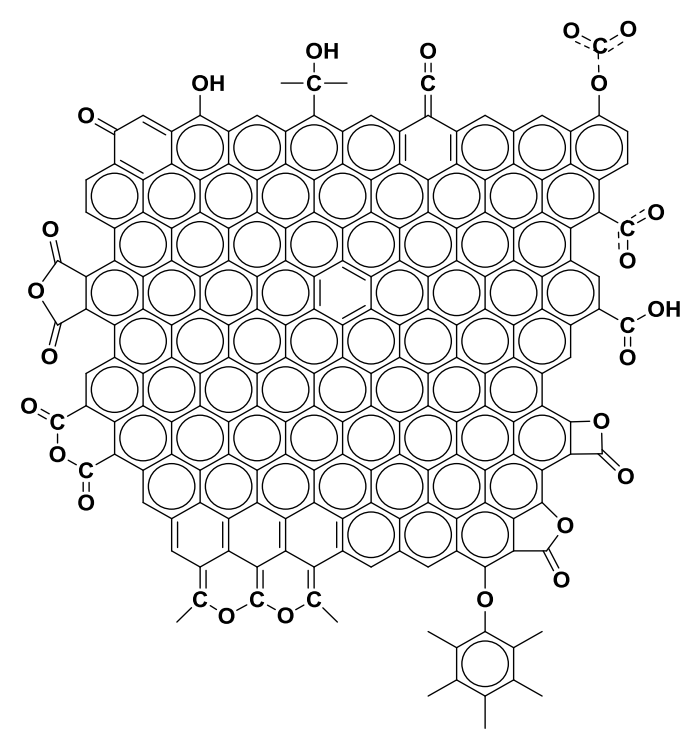

Figure 19 - Possible functional groups on the CB-oxidized surface suggested by Fanning. Adapted with permission from ref [113]. Copyright 1993 Elsevier. 


\subsubsection{Gaseous oxidation}

In 1956, Smith et al., reported the oxidation of fine thermal $\mathrm{CB}$ at $600{ }^{\circ} \mathrm{C}$ under air flow, and consequent six fold increase in its surface area [116]. A similar procedure was reported by Heckmann et al. in 1966 in which hot air progressively oxidized CB particles (BLACK PEARLS® 2000), thus introducing oxygen mainly on the accessible portion of CB surface [113]. Chen et al. further compared both air-oxidized and ozone-treated CBs, finding differences between $\mathrm{O}_{3}$ and $\mathrm{O}_{2}$ selectivity for preferential sites. It was found that $\mathrm{O}_{2}$ is more selective towards the $\mathrm{BSU}$ crystallite edges, while $\mathrm{O}_{3}$ tends to homogeneously attack the whole surface. However, $\mathrm{O}_{2}$ caused a significant increase in surface area as compared to $\mathrm{O}_{3}$ [117]. Later studies in 2009 using air oxidation at $300{ }^{\circ} \mathrm{C}$ for acetylene blacks of different size, i.e., $12 \mathrm{~nm}$ and $35 \mathrm{~nm}$, yielded oxidized graphene sheets protruding from the surfaces of the spherical CB particles [118].

\subsubsection{Wet oxidation}

Wet $\mathrm{CB}$ oxidation relies on several oxidizing agents, ozone $\left(\mathrm{O}_{3}\right)$ in aqueous medium [119], nitric acid $\left(\mathrm{HNO}_{3}\right)$ [120, 121], hydrogen peroxide $\left(\mathrm{H}_{2} \mathrm{O}_{2}\right)$ [121-123], ammonium persulfate, $\left(\mathrm{NH}_{4}\right)_{2} \mathrm{~S}_{2} \mathrm{O}_{8}$, in sulfuric acid [124], and ammonium persulfate in $\mathrm{HCl}$ [125], have all been effectively used to oxidize $\mathrm{CB}$ with varying results.

Overall, all these methods tend to affect the CB textural properties, e.g., surface structure and morphology, yielding higher or lower specific surface areas by introducing or blocking access to existing micropores, respectively. Nonetheless, Sosa and co-workers reported no change in the textural properties of $\mathrm{CB}$ through treatment with $\mathrm{H}_{2} \mathrm{O}_{2}$ or $\mathrm{HNO}_{3}$. Upon $\mathrm{H}_{2} \mathrm{O}_{2}$ or $\mathrm{HNO}_{3}$ treatment, the surface charge of $\mathrm{CB}$ became more negative, in contrast to gaseous thermal oxidation with a $\mathrm{He} / \mathrm{O}_{2}$ mixture that had the opposite effect [121]. Notably, the reproducibility of oxidation reactions remains controversial resting on numerous contributing factors such as reaction time and acid concentration. Besides oxidation time, comparison of the different $\mathrm{CB}$ subtypes, e.g., furnace black (FB) and acetylene black (AB), the oxidation percentage can be dramatically affected, as presented in Table 2 for comparative oxidation of both $\mathrm{AB}$ and $\mathrm{FB}$ in $\mathrm{HNO}_{3}$. In their study, Kamegawa and co-workers found that the oxygen contents of oxidized acetylene black (ABO) were remarkably lower than those of oxidized furnace black (FBO). The authors related these discrepancies to the lower number of edge sites in the BSU of $\mathrm{AB}$ compared to FB. In other words, FB has smaller and more fragmented $\mathrm{BSU}$ than $\mathrm{AB}$, and consequently a greater availability of edge sites than $\mathrm{AB}[8]$. 
In $\mathrm{AB}$, larger graphene layers stacked in greater numbers lower the BSU surface area and resulting in fewer accessible edge sites. Whereas in FB, BSU with smaller basal planes stacked in lower numbers increase the amount of edge sites and reactivity of CB.

Despite the lack of exact surface models to describe the oxidation of graphitic carbons, the formation of oxidized fractions similar to humic acids [126] and the presence of mellitic acid as a by-product [127], indicate the extensive addition of multiple carboxylic and oxygenbearing functional groups upon oxidation. In general, the nitric acid treatment of $\mathrm{CB}$ produces a soluble mixture of small polyaromatic species with a high content of mellitic acid. It is noteworthy that nitrogen is also detected in the product of oxidation. It is likely the nitration reaction also occurs leading to the formation of $\mathrm{C}-\mathrm{NO}_{2}$ or its isomer $\mathrm{C}-\mathrm{O}-\mathrm{N}=\mathrm{O}$.

Table 2 - Effect of CB grade and reaction time on the elemental content for oxidation in $\mathrm{HNO}_{3}$.

\begin{tabular}{ccccccc}
\hline \multirow{2}{*}{ CB } & $\begin{array}{c}\text { Oxidation } \\
\text { time (h) }\end{array}$ & \multicolumn{5}{c}{ Element content (\%) } \\
& & $\mathrm{C}$ & $\mathrm{O}$ & $\mathrm{H}$ & $\mathrm{N}$ & Ash \\
\cline { 3 - 7 } Pristine FB & - & 97.1 & 2.6 & 0.1 & 0.2 & 0.4 \\
Pristine AB & - & 99.3 & 0.3 & 0.0 & 0.4 & 0.1 \\
FBO & 20 & 73.3 & 25.2 & 0.5 & 1.0 & 0.2 \\
FBO & 100 & 57.9 & 38.6 & 0.9 & 2.6 & 0.2 \\
FBO & 200 & 58.6 & 38.5 & 0.9 & 2.0 & 0.1 \\
ABO & 25 & 97.3 & 2.2 & 0.0 & 0.5 & 0.2 \\
ABO & 100 & 96.2 & 3.4 & 0.0 & 0.4 & 0.2 \\
ABO & 200 & 94.6 & 4.9 & 0.0 & 0.5 & 0.1 \\
\hline
\end{tabular}

Oxidized CB is an excellent platform to make graphene-based materials. Heavily $\mathrm{HNO}_{3}-$ oxidized CB (CORAX® N110) was converted to self-assembled graphene-like films using hydrazine hydrate as a reducing agent. In the same study, authors reported this approach to be more advantageous for production of water-insoluble graphene-like films over the conventional graphite oxidation and reduction method, mainly in terms of using milder conditions required for CB (Figure 20) [128]. 


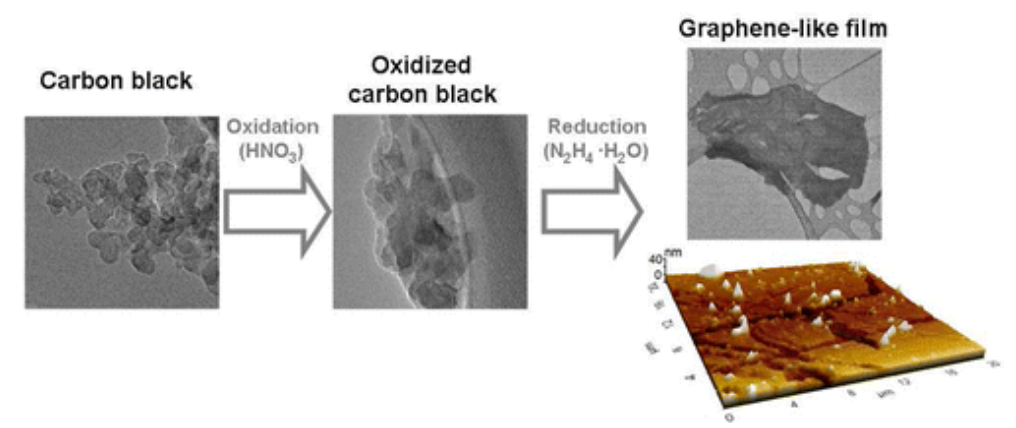

Figure 20 - Producing graphene-like films from CB. Reprinted with permission from ref [128]. Copyright 2012 American Chemical Society.

It is noteworthy that $\mathrm{H}_{2} \mathrm{O}_{2}$ is considered a more environmentally-friendly agent as it produces no hazardous emissions [129], compared to noxious gases emitted during oxidation with $\mathrm{HNO}_{3}$ (dark orange vapors related to $\mathrm{NOx}$ ) and $\mathrm{O}_{2}$ (yellow vapors related to volatile degradation products) [121]. Assuming that the reaction of $\mathrm{CB}$ with $\mathrm{HNO}_{3}$ would proceed similarly to what has been proposed for soot and graphitic carbons (CNT, graphite, fullerene, etc.) [130-133], a possible mechanism of $\mathrm{CB}$ oxidation with $\mathrm{HNO}_{3}$ is illustrated in Figure 21. Two molecules of $\mathrm{HNO}_{3}$ interact with each other providing the surface with the required oxygen, with subsequent $\mathrm{H}_{2} \mathrm{O}$ and $\mathrm{NOx}$ evolution as by-products of $\mathrm{HNO}_{3}$ treatment. $\mathrm{CO}_{2}$ and $\mathrm{CO}$ are released from the surface as a result of $\mathrm{CB}$ degradation [130]. In addition, a low content of nitrogen is also detected as a consequence of the introduction of nitro groups [128].
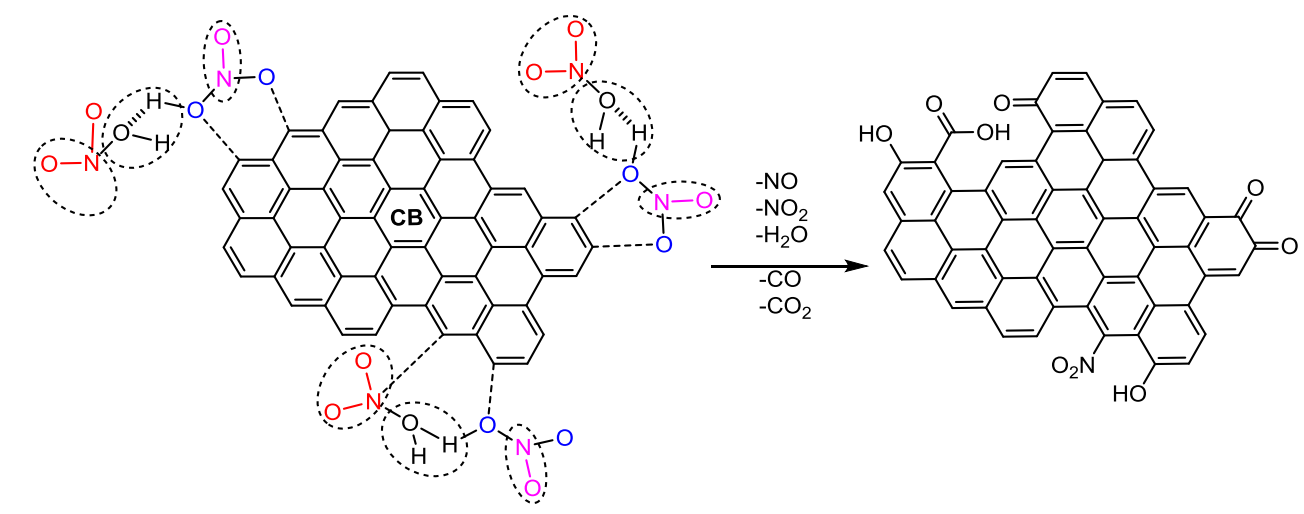

Figure 21 - The oxidation mechanism of $\mathrm{CB}$ using $\mathrm{HNO}_{3}$ and formation of representative functional groups.

The oxygen content added to the final product is dependent on the reaction time, longer $\mathrm{HNO}_{3}$ treatment introduces more oxidized functions. This allows the favorable tuning and control of the oxidation level, but there are still some drawbacks associated with $\mathrm{HNO}_{3}$ 
corrosivity and difficult recycling, this method has become the most practical and common way to prepare oxidized $\mathrm{CB}$ (OCB) with high oxygen content for many $\mathrm{CB}$ grades (except acetylene black).

An alternative wet oxidation method is the Hummers-based oxidation. The Hummers oxidation has drawn considerable attention in recent years, as it is the method of choice for the oxidation of graphite to obtain graphite oxide [134], a precursor to graphene oxide (GO), and to reduced graphene oxide (rGO), or simply graphene $[135,136]$. It is also recognized as the most efficient approach to generate $\mathrm{OH}$ and $\mathrm{COOH}$ moieties in carbons, as well as epoxy groups on graphene layers [137]. Although this method has been extensively modified through alteration of reaction conditions, in general, it relies on the addition of potassium permanganate $\left(\mathrm{KMnO}_{4}\right)$ to a solution of graphite, sodium nitrate, and sulfuric acid. Similar to graphite, CB undergoes oxidative peeling using the Hummers method as it has been studied by Wilson et al. (Figure 22) [18].

It has been hypothesized that outer layers of carbon particles "peel off" because these are oxidized while the inner cores remain mostly intact, with enough oxidation to provide for a significant solubility in water only for the peeled layers. The oxidation products can be separated by centrifugation, both particles and peeled layers. In fact, the exact structure of the peeled by-product layers remains unknown. One could presume they are similar to small polyaromatic compounds/mellitic acid-containing fraction described in earlier literature [120]. Nonetheless, blue photoluminescence is one of the main properties exhibited by the peeled-off shells, characteristic to (GO)-like nanosheets (Figures 10 and 22) [18]. Despite the high efficiency of the Hummers method due to its high levels of oxidation, this approach suffers from using hazardous chemicals, harsh acidic conditions, and cumbersome reactionwork up making it less popular and practical for upscaling CB oxidation. 

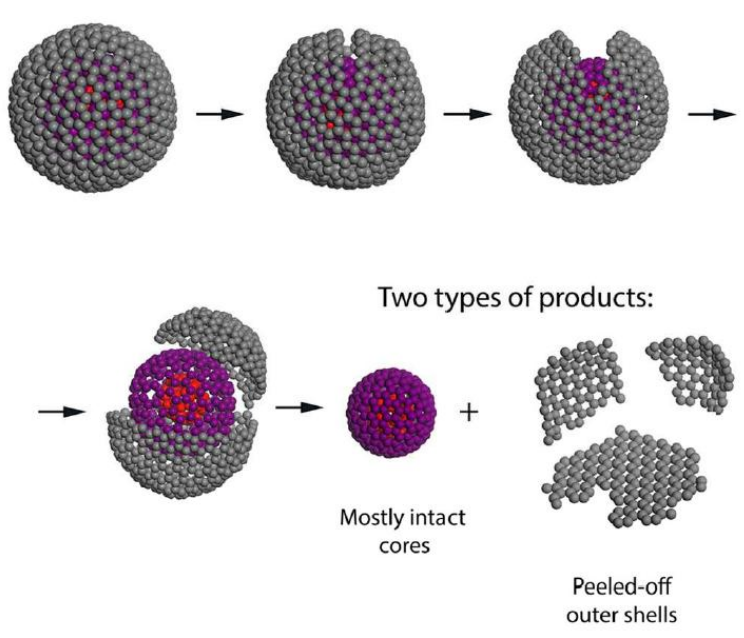

Figure 22 - The suggested mechanism of the oxidative peeling of CB NPs through which the peeled graphene-oxide-like sheets are obtained from the outer shells and lightly oxidized cores. Reprinted with permission from reference [18]. Copyright 2015 Royal Society of Chemistry.

Another wet oxidation approach consist in the use of a piranha solution, a mixture of 3:1, or even as high as 7:1 parts of sulfuric acid and hydrogen peroxide, respectively, has been effectively used for surface oxidation of CB. This metal-free procedure yields oxidized CB (c-nergy super C65) with no appreciable change in physical appearances compared to starting CB for a 3:1 v/v $\mathrm{H}_{2} \mathrm{SO}_{4}: \mathrm{H}_{2} \mathrm{O}_{2}$ mixture [138]. Alternatively, a $\mathrm{H}_{2} \mathrm{O}_{2}: \mathrm{H}_{2} \mathrm{SO}_{4}$ ratio of 5:1 w/w was used to promote the oxidation of high-surface-area $\mathrm{CB}$, leading to a higher degree of oxidation $(\mathrm{O} / \mathrm{C}=0.66)$ [139]. This approach, however, is less effective on low surface area CB. Another drawback is that the resulting OCBs contain sulfur as contamination, similarly to the OCBs prepared with the Hummers method.

\subsection{Surface modification of oxidized carbon black}

After oxidation, the surfaces of $\mathrm{CB}$ become rich in oxygen functionalities, among which carboxylic groups comprise an appreciable fraction [139-142]. As a result, the further modification of the surface of OCB is related to the chemistry and reactivity of the carboxyl and quinonyl groups [132], but mainly $-\mathrm{COOH}$ functions independent of $\mathrm{CB}$ grade. The amount of $\mathrm{COOH}$ groups has been determined in $\mathrm{OCB}$ obtained from $\mathrm{HNO}_{3}$ treatment as 5.1-6.7 $\mathrm{mmol} \mathrm{g}^{-1}$ [120]. The carboxylic groups on the oxidised REGAL ${ }^{\circledR} 400$ can be easily converted into acyl chloride (-COCl) with common chlorinating agents such as thionyl [143] 
or oxalyl chloride [144]. Replacing $\mathrm{OH}$ with $\mathrm{Cl}$ as the leaving group allows the acyl chloride groups to further undergo condensation reactions with appropriate nucleophiles, thus providing a platform to make a wide variety of hybrid materials. Alternatively, various coupling agents including 4-(4,6-dimethoxy-1,3,5-triazin-2-yl)-4-methylmorpholinium chloride (DMTMM) and dicyclohexylcarbodiimide (DCC), and 1-ethyl-3-(3dimethylaminopropyl) carbodiimide (EDCI) [142] have been utilized under milder reaction conditions. Depending on the selected reaction conditions, the degree of surface functionalization and final surface properties can be tailored [143]. This is schematically represented in Figure 23, where Method 1 using DMTMM as a coupling agent, and Method 2 using thionyl chloride $\left(\mathrm{SOCl}_{2}\right)$, yielded surface modification ratios of $47 \%$ and $92 \%$, respectively. The degree of functionalization was determined by titration of unreacted $\mathrm{COOH}$ groups. A consequence of the different degree of surface modification was that Method 2 resulted in $\mathrm{CB}$ with increased surface hydrophobicity as compared to OCB or the product of Method 1.

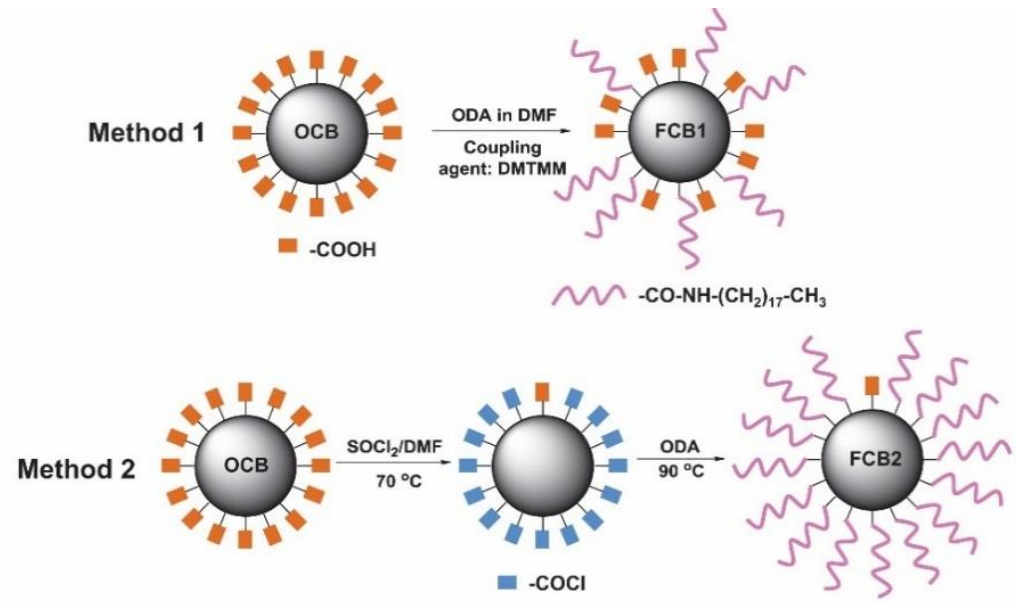

Figure 23 - Surface functionalization of OCB with octadecyl amine (ODA). Reprinted with permission from reference [143]. Copyright 2014 Wiley-VCH.

In addition to alkane surface groups, Table 3 lists a number of alkyl/aryl amines, alcohols, and polymers that have been investigated for the modification of OCB in the presence of various coupling agents.

Table 3 - Reaction of the carboxyl groups of OCB with nucleophiles.

\begin{tabular}{|l|l|l|l|}
\hline Reactant & Reagent & Product & Ref. \\
\hline
\end{tabular}




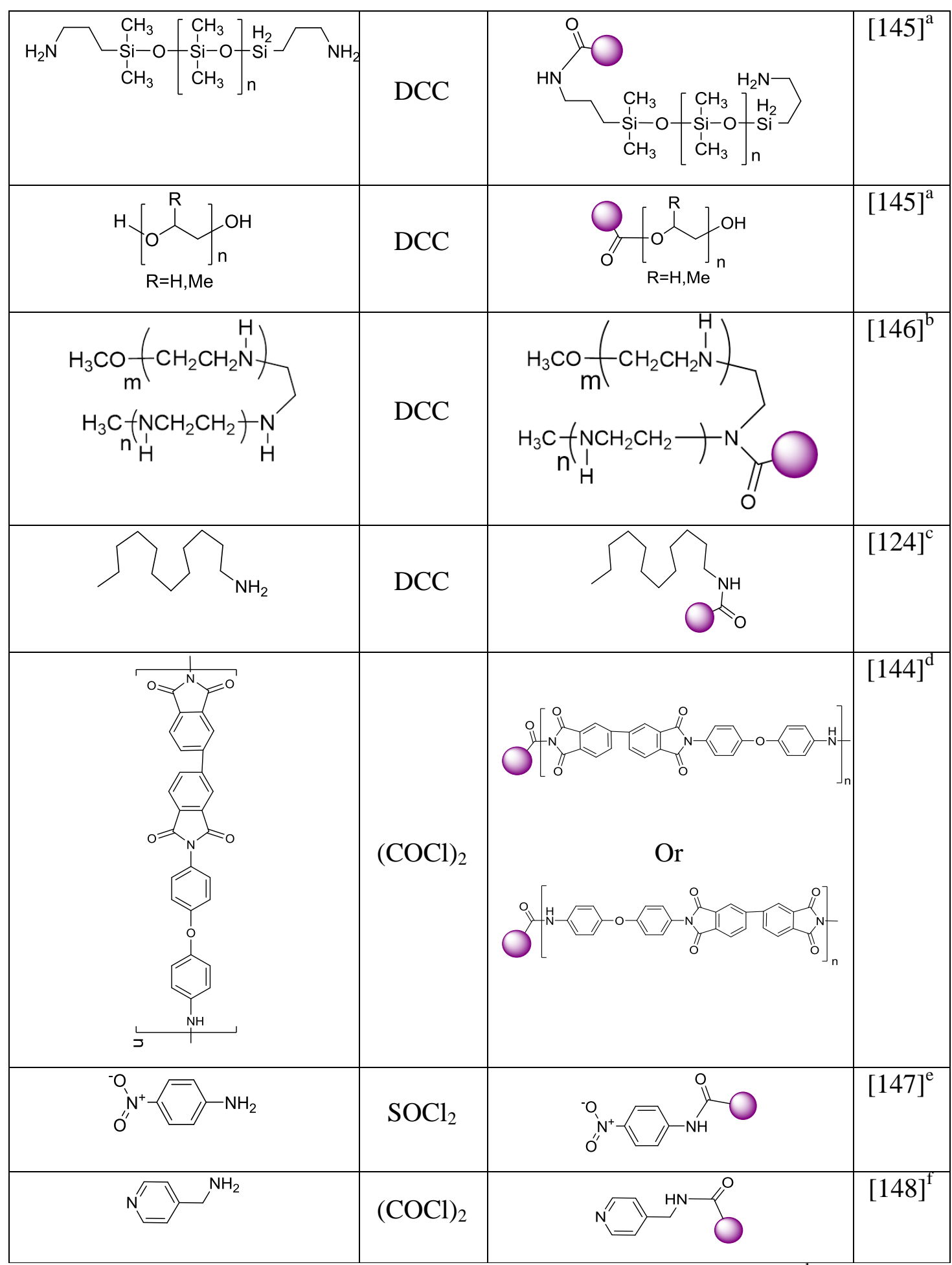

${ }^{\mathrm{a} N e o}$ Spectra II, COLOUR BLACK FW 200, and Philblack 0 (N330). ${ }^{b}$ Furnace Black, Porousblack. ${ }^{\mathrm{c}} \mathrm{MONARCH}{ }^{\circledR}$ 1100. ${ }^{\mathrm{d}} \mathrm{MOGUL}^{\circledR}$ L. ${ }^{\mathrm{e}} \mathrm{MONARCH}^{\circledR}$ 120, 430, and 1100. ${ }^{\mathrm{f}} \mathrm{VULCAN}^{\circledR} \mathrm{XC}-72 \mathrm{R}$.

Other functionalities present on the surface of $\mathrm{OCB}$ are $\mathrm{OH}$ containing organic moieties. In a few reports, the hydroxylic group has been found to be a good nucleophile able to react with 
the electrophilic carbonyl carbon of carboxylic acids, thus allowing for the esterification reaction between $\mathrm{CB}$-modified with $\mathrm{OH}$ containing moieties and carboxylic acids. By using suitable unsaturated acids, OCB (PRINTEX ${ }^{\circledR}$ L and NEROXTM 600) nanoparticles were first esterified with oleic acid further subjected to miniemulsion (or microemulsion) polymerization with styrene $[149,150]$ as schematically represented in Figure 24.

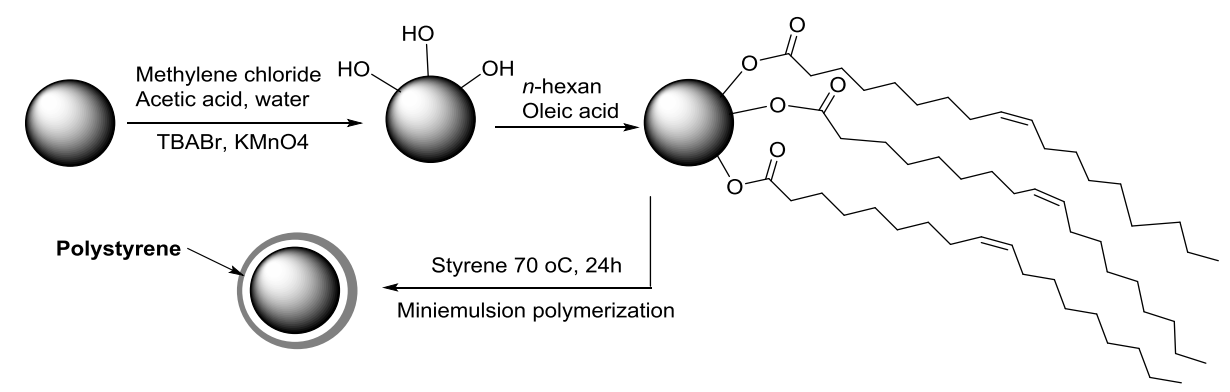

Figure 24 - Preparation of the polystyrene-microencapsulated CB through oxidation, esterification, and miniemulsion polymerization $(\mathrm{TBABr}=$ tetrabutyl ammonium bromide $)$. Adapted with permission from reference [149]. Copyright 2010 Elsevier.

\subsection{Covalent functionalization by direct carbon-carbon bond formation}

An effective strategy to $\mathrm{CB}$ functionalization it the use of aryl diazonium salts. As can be seen in Figure 25, in situ generated aryl diazonium salts produced from aniline derivatives after treatment with sodium nitrite have been added into the structure of CB (VULCAN® XC-72R and VULCAN® 9 N115) through a radical substitution mechanism. A highly agreed assumption is that the carbon $\pi$-electron system is likely to play the nucleophilic role transferring electrons required to reduce the diazonium cations. This resulted in the covalent C-C bonding of a variety of aryl units with the CB surface $[151,152]$.

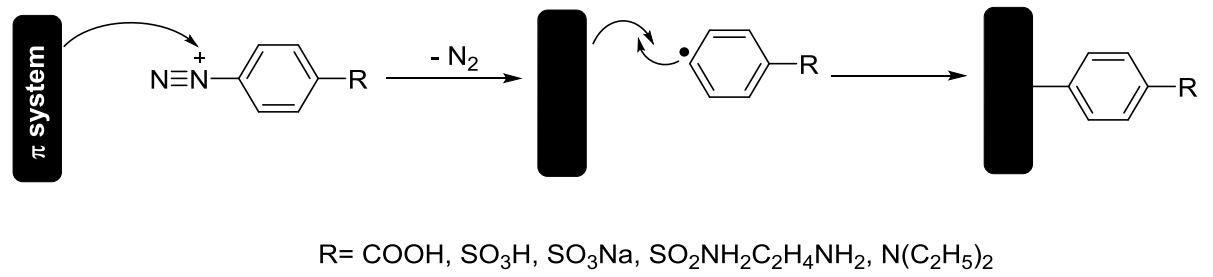

Figure 25 - Heterolytic C-C bond formation between the CB surface ( $\pi$ system) and the phenyl radical. Adapted with permission from reference [151]. Copyright 2008 American Chemical Society. 
Furthermore, diaryl diazo compounds have also been employed to functionalize MONARCH $^{\circledR} 430$ for use in polypropylene nanocomposites [153]. The proposed mechanism for this reaction follows a thermal diazonium coupling reaction through the formation of transient carbene produced by the decomposition of the labile diazo precursor (Figure 26).
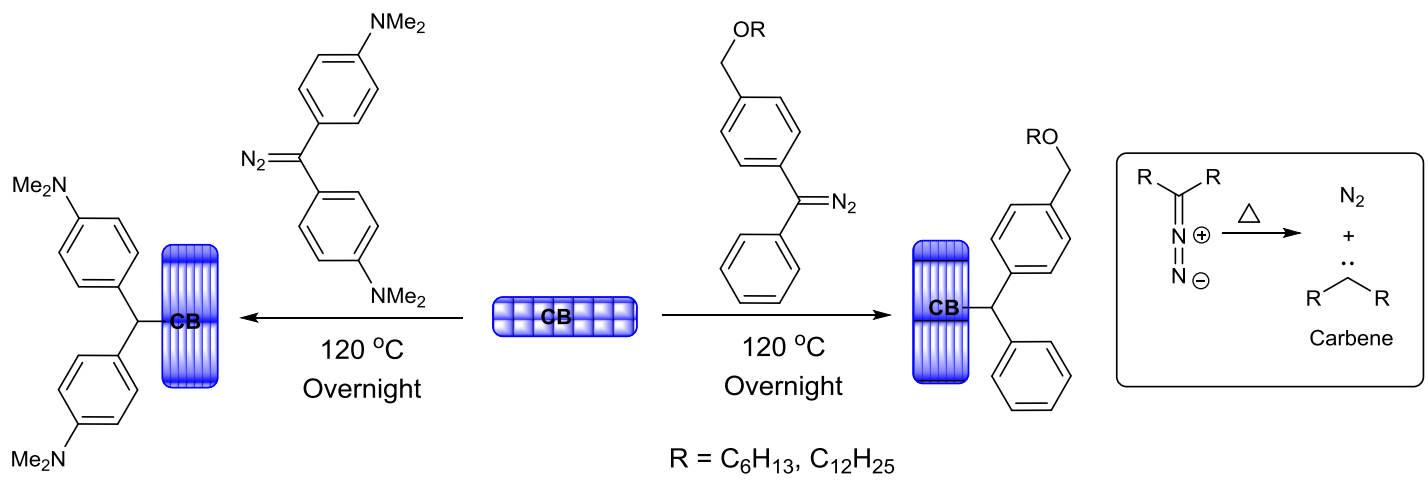

Figure 26 - Carbene insertion onto $\mathrm{CB}$ by diazonium coupling reactions. The formation of carbene is shown on the right. Adapted with permission from reference [153]. Copyright 2016 American Chemical Society.

Another covalent functionalization approach is based on Diels-Alder reactions. The addition of maleic acid derivatives to CB has been first reported by Bergemann in 2004 [154]. The reaction follows the Diels-Alder mechanism where a diene and dienophile are involved in a $2+4$ cycloaddition (Figure 27). It is believed that diene structures existing in the aromatic network of CB can react with reactive dienophiles. Depending on the melting or sublimating point of the dienophile, both solution (anisole, toluene, and water as solvent) and solid-state method can be used for this purpose, subsequently yielding from 25 to $90 \mathrm{wt}$.\% conversion.

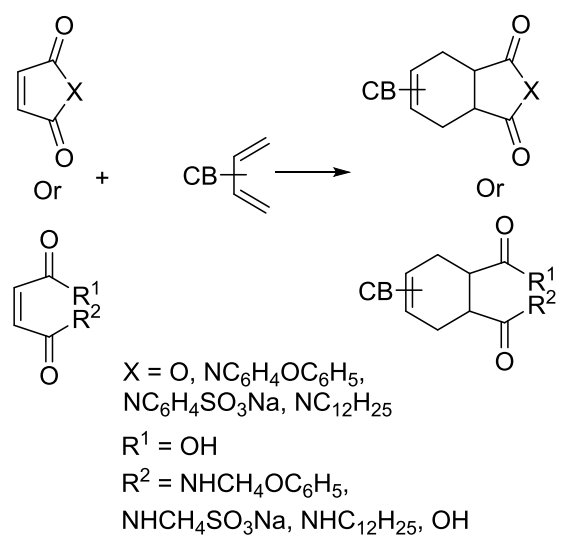

Figure 27 - Reaction of the diene part of $\mathrm{CB}$ with maleic acid derivatives. Adapted with permission from reference [154]. Copyright 2004 Elsevier. 
Radical reactions are also versatile methods to introduce covalent functionalization to aromatic compounds. Aromatic rings have a high methyl radical affinity which allows polycondensation reactions involving non-aromatic alkyl radicals including those formed from polymers $[155,156]$. CB has also proven ability to act as a strong radical scavenger, either through the quinone oxygen groups or through hydrogen atoms bound to unsaturated edge carbon atoms from the poly-condensed aromatic rings [132]. This reaction is exemplified by the polymer grafting mechanism onto $\mathrm{CB}$, where the poly-condensed aromatic rings of $\mathrm{CB}$ trap the macroradicals. Given the reactivity of $\mathrm{CB}$, the following radical reaction mechanisms have been investigated for surface functionalization: (i) polymer grafting and encapsulation, (ii) atom transfer radical polymerization, both discussed in Section 3.4, and (iii) radical reaction with small molecules.

Radical reactions with small molecules have been used to introduce perfluorinated groups on the surface of $\mathrm{CB}$. Covalent linkage of perfluorocarbon and perfluoropolyether chains on the CB surface were achieved via the reaction of VULCAN ${ }^{\circ} \mathrm{XC}-72 \mathrm{R}$ with carbon centered perfluorinated free radicals. The latter were generated by the thermolysis of perfluorodiacyl and perfluoropolyether peroxides [157]. It is worth noticing that this approach provided a highly hydrophobic $\mathrm{CB}$, further enhancing its hydrorepellence, while the textural properties of the modified $\mathrm{CB}$ were mostly retained. As a representative reaction, Figure 28 illustrates the perfluorination of $\mathrm{CB}$ by perfluoropolyether peroxides.

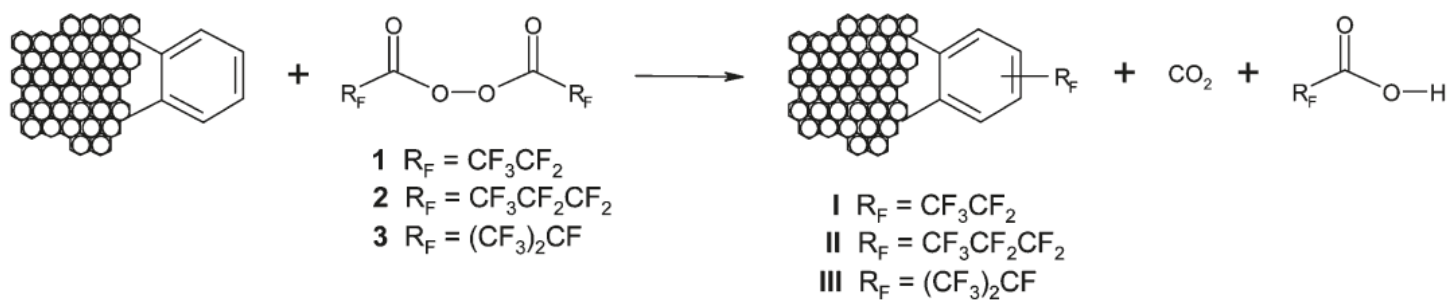

Figure 28 - Schematic of the reaction of CB with perfluorodiacyl peroxide. Reprinted with permission from reference [157]. Copyright 2009 American Chemical Society.

\subsection{Functionalization with polymers}

Polymer grafting and encapsulation have been successfully applied to CB. Encapsulation and grafting follow two distinct mechanisms, but some authors have interchangeably used the two terms. A comprehensive review by Tsubokawa on the functionalization of CB by surface grafting of polymers was reported in the early 90's, no other reviews have appeared on the 
later progress and findings since then [158]. Therefore, we report here some of the later most outstanding findings in the area of polymer grafting on CB.

Tsubokawa et al. proposed three distinct mechanisms for grafting polymers on CB. These proceeded through anionic, cationic, and living radical polymerization. These reactions have been classified as (a) Grafting onto $C B$ (i.e., CB-X + growing polymer $\rightarrow \mathrm{CB}-\mathrm{X}$-polymer, with $\mathrm{X}=$ functional group on $\mathrm{CB}$ that grafts the polymer chain and stops its growth), (b) Grafting from $C B$ (i.e., $\mathrm{CB}-\mathrm{X}+$ monomer $\rightarrow \mathrm{CB}-\mathrm{X}$-polymer, with $\mathrm{X}=$ functional group on $\mathrm{CB}$ that initiates the polymerization of the monomer), and (c) Reaction of $C B$ with polymer (i.e., $\quad \mathrm{CB}-\mathrm{X}+\mathrm{Y}$-polymer $\rightarrow \mathrm{CB}$-Z-polymer, with $\mathrm{X}+\mathrm{Y}=\mathrm{Z}$ representing the reaction between the functional groups $\mathrm{X}$, of $\mathrm{CB}$, and $\mathrm{Y}$, of the polymer).

2,2,6,6-tetramethyl-1-piperidinyloxy (TEMPO)-terminated polystyrene (PSt-TEMPO) was employed by the Tsubokawa group to graft polystyrene with controlled and narrow molecular weight distribution onto CB (Philblack I and COLOUR BLACK FW 200). This method yielded PSt-grafted CB as a stable colloidal dispersion in THF. Briefly, the polymer radicals formed from the thermal dissociation of the C-ON bond of PSt-TEMPO grafted onto the poly-condensed aromatic rings of $\mathrm{CB}$. While the polystyrene radical reacted with $\mathrm{CB}$, the TEMPO radical was not trapped on the CB surface, as presented in Figure 29 [159].

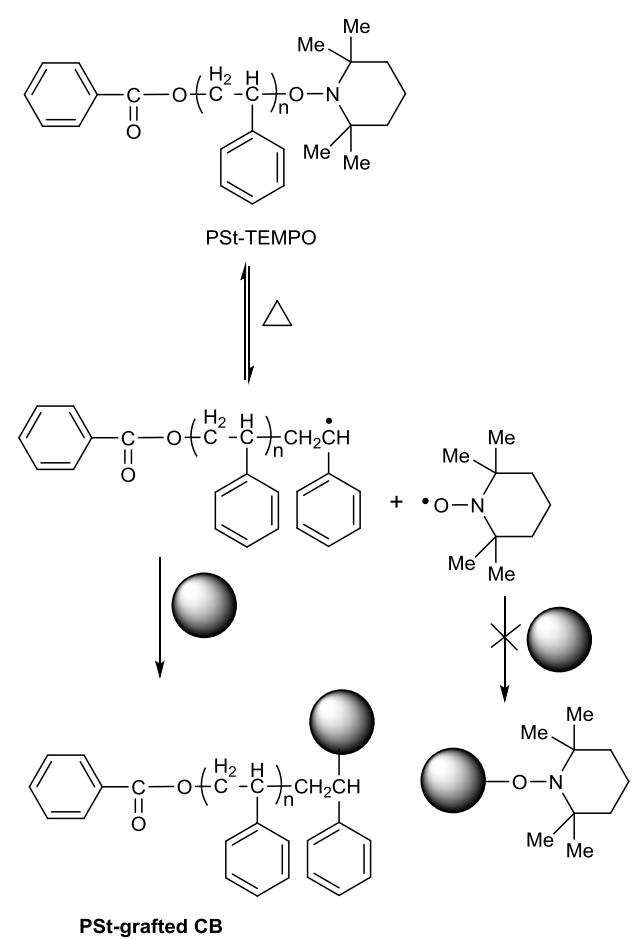

Figure 29 - Grafting polystyrene onto CB. Adapted with permission from reference [159]. Copyright 1998 Wiley-VCH. 
The presence of delocalized radicals during the reaction of PSt-TEMPO and CB was confirmed by electron spin resonance. The radicals, however, may attack either (i) a carbon at the edge of the aromatic graphene system, or (ii) quinonic oxygen groups, thus illustrating the impact of the surface heterogeneity on this reaction, as shown in Figure 30. These two mechanisms are of general value and valid for other radical scavenging reactions.

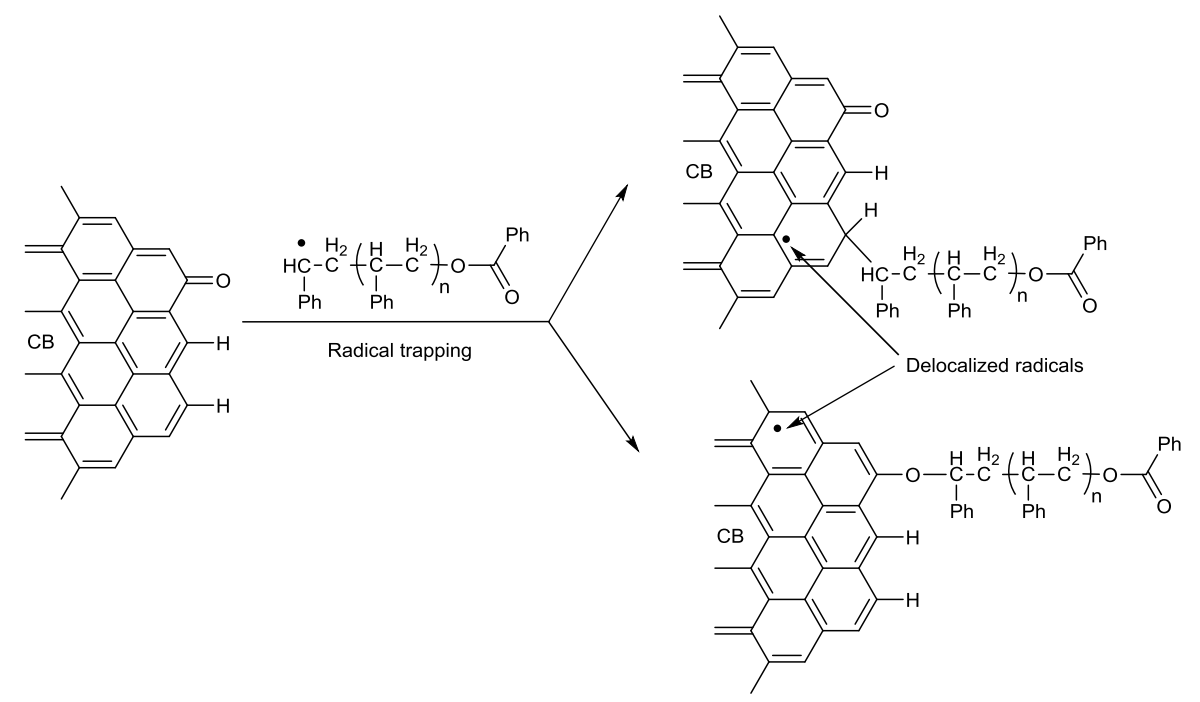

Figure 30 - Possible mechanism of scavenging of the radical molecules by CB. Adapted with permission from reference [159]. Copyright 1998 Wiley-VCH.

Atom Transfer Radical Polymerization (ATRP) can also be used to covalently attach polymers to $\mathrm{CB}$. ATPR has become one of the most widely investigated living radical polymerization methods. It has been used to develop a broad range of polymers with precisely controlled molecular weight, functionality and nanostructure [160, 161]. Figure 31 presents the multi-step modification of CB (MONARCH 700) recently carried out using ATRP [162-166]. CB modified with $\mathrm{C}_{6} \mathrm{H}_{4} \mathrm{COONa}$ groups (a) was used as the starting material. Once protonated (b), and in some cases further converted to acyl chloride (c), ATRP initiators, e.g. $\alpha$-bromoisobutyrate groups, were anchored onto the surface providing the $\mathrm{CB}-\mathrm{Br}$ platform substrate ( $\mathrm{d}$ and e) on which to grow the target polymers [164]. For example, $n$-butyl acrylate ( $n$-BA) was grown onto this substrate to give PBA (f) densely grafted on CB aggregates [163]. Another example is the grafting of 2-(dimethylamino)ethyl methacrylate (DMAEMA) in methanol to give copolymer functionalized $\mathrm{CB}$ ( $\mathrm{g}$ ) further modified to its ammonium form through quaternization with ethyl bromide (h). The CB-Br substrate (e) was also used to make $\mathrm{CB}-\mathrm{P}(n-\mathrm{BA})$ (i), equivalent to (f), on which to 
polymerize t-butyl acrylate (t-BA) (j) [165]. The t-butyl groups of the resulting P(t-BA) polymeric segments were then removed using a strong acid $\left(\mathrm{CF}_{3} \mathrm{COOH}\right)$ to convert the $\mathrm{P}(\mathrm{t}-$ BA) to poly(acrylic acid), P(AA) (k). The carboxylic groups of the $\mathrm{P}(\mathrm{AA})$ were subsequently deprotonated in aqueous $\mathrm{NaOH}$ solution to provide water dispersible $\mathrm{CB}$ (1). In these examples, the molecular weight of the polymers was controlled using sacrificial initiators such as ethyl 2-bromoisobutyrate typically used in surface-initiated controlled radical polymerizations [162].

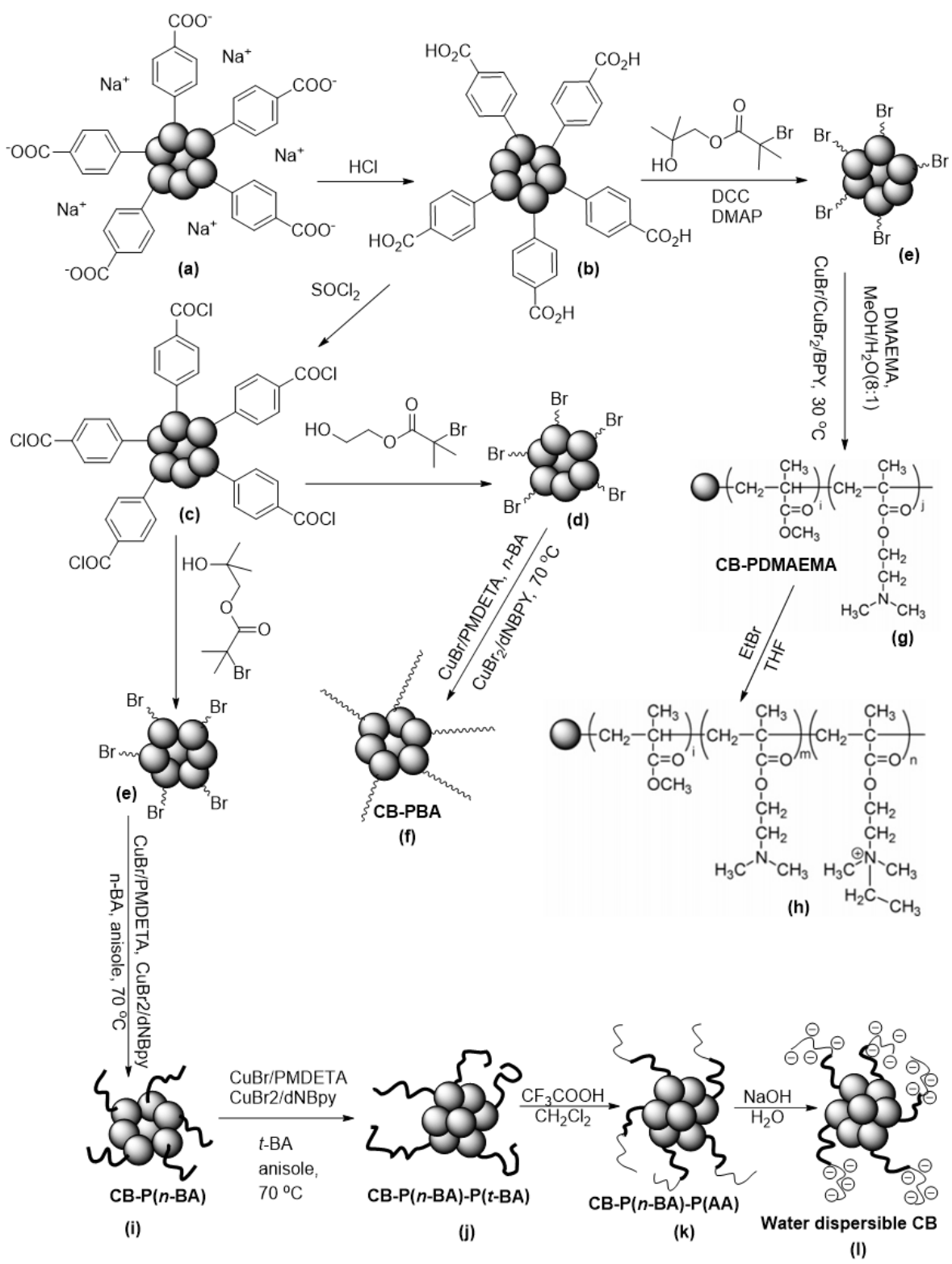

Figure 31 - Multi-step manipulation of CB by ATRP. Redesigned and adapted with permission from references [162-165]. Copyright 2002, 2003, 2006 American Chemical Society, and 2005 Wiley-VCH. 
CB-Br substrates for ATRP polymerization have also been synthesized following two other methods (Figure 32). The first is similar to what seen previously in Figure 31, reaction from (c) to (d), and consists in the reaction of acyl chloride modified CB with 2-hydroxyethyl 2bromoisobutyrate. However, in this case the $-\mathrm{COOH}$ groups necessary to introduce the acyl chloride on $\mathrm{CB}$ were initially added via wet oxidation with $\mathrm{HNO}_{3}$. The second method, instead, uses nitrene chemistry to place both azido and bromine groups directly onto the $\mathrm{CB}$ surface. In both cases, the CB surface was then functionalized with hyperbranched polymers, such as poly(p-chloromethylstyrene) (PCMS) [166].

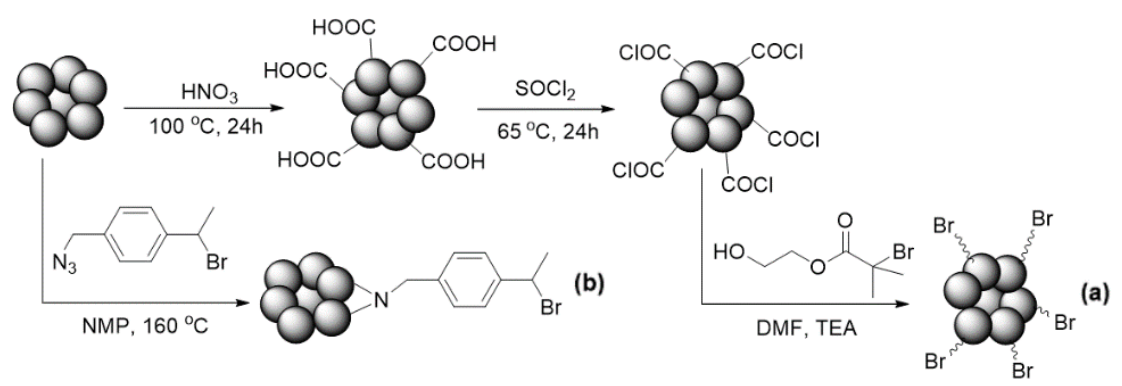

Figure 32 - Functionalization of CB with ATRP initiator by (a) acid oxidation method and (b) nitrene chemistry method. Redesigned and adapted with permission from reference [166]. Copyright 2013 Royal Society of Chemistry.

Figure 33 shows different synthetic routes to produce polymer core-shell structures. In these examples, pristine $\mathrm{CB}$ (VXC 605) was first oxidized with $\mathrm{HNO}_{3}$ to introduce carboxylic groups onto the $\mathrm{CB}$ surface, the resulting $\mathrm{OCB}$ was then treated with thionyl chloride $\left(\mathrm{SOCl}_{2}\right)$. In the following step, glycol was introduced, thus providing the substrate (a) on which to grow various polymers-CB core-shell structures. For instance, the core-shell structure (b) [167] was prepared from the surface-initiated ring-opening polymerization of $\varepsilon$ caprolactone. Similar studies led to the anchoring of $\alpha$-bromoisobutyrate groups ATRP initiators (c) from the reaction of 2-bromo-2-methylpropionyl bromide with (a). Thereupon, polystyrene (d), poly(methyl methacrylate) (e) [168], and poly( $N$-isopropylacrylamide) (f) [169], chains were grafted on the CB surface by surface-initiated ATRP. 


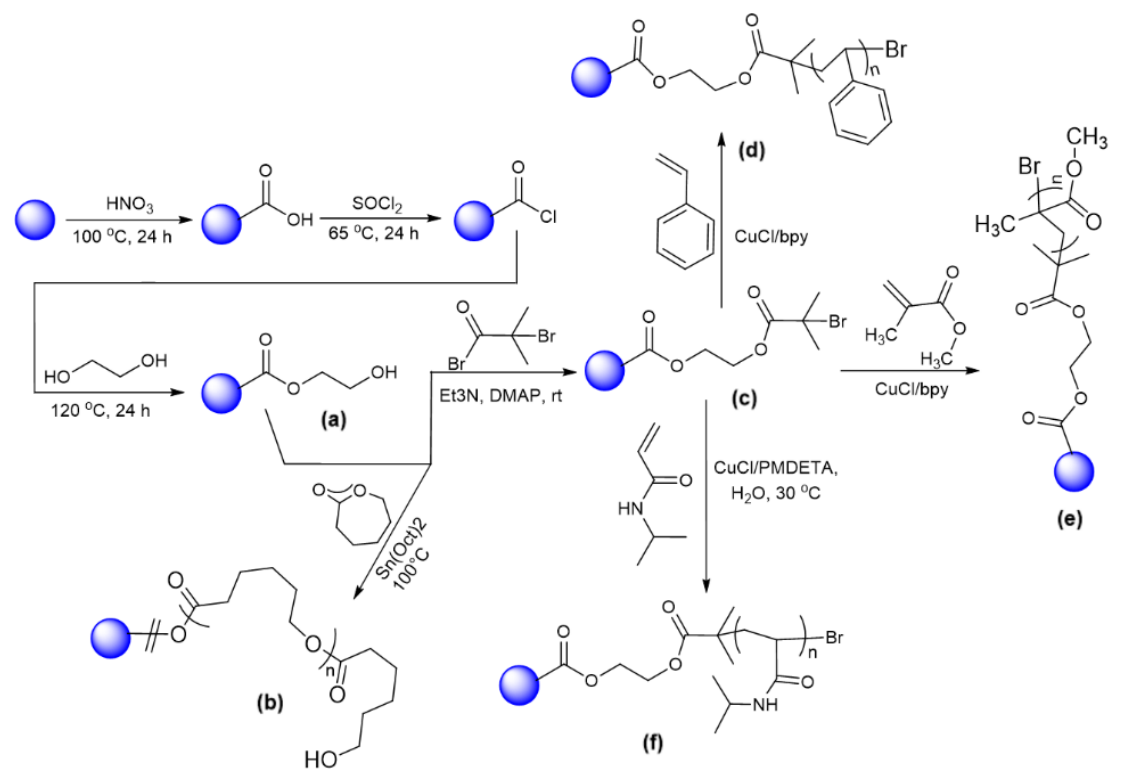

Figure 33 - Preparation of hyperbranched polymers or core-shell structures by ATRP initiator. Redesigned and adapted with permission from references [167-169]. Copyright 2007 Elsevier, 2007 and 2008 Wiley-VCH.

Polymer-modified carbon black was also prepared by Hwang et al. who synthesized sulfonated polyvinyl alcohol functionalized CB nanocomposites and tested them as downhole hydrocarbon detectors for oil and gas exploration (Figure 34). The sulfonate groups enhanced the thermal and chemical stability of the $\mathrm{CB}$ nanoparticles required for the high temperatures and high salinity conditions found in the downhole rock environment [170].

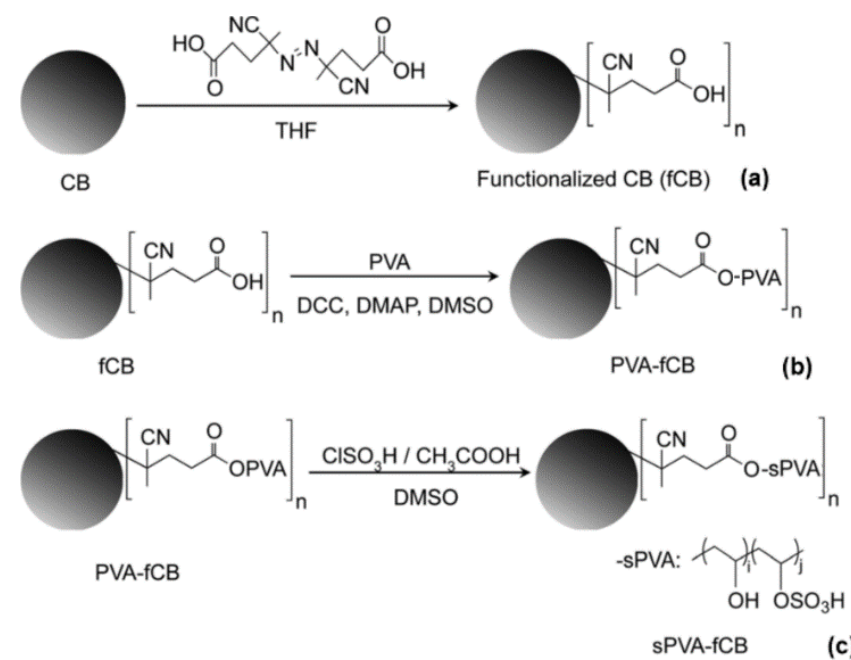

Figure 34 - Synthesis of (a) functionalized carbon black (fCB); (b) PVA modified fCB (PVA-fCB) and (c) sulfated PVA-fCB (sPVA-fCB). Sulfonation yields units with $-\mathrm{OSO}_{3} \mathrm{H}$ 
pendants. Reprinted and adapted with permission from reference [170]. Copyright 2012 Royal Society of Chemistry.

Table 4 is a summary of the most relevant CB-polymer products obtained from the grafting of polymer radicals onto $\mathrm{CB}$. In these examples, all polymer radicals were formed via thermal activation, with subsequent polymer grafting onto the $\mathrm{CB}$ surface.

Table 4 - Overview of polymer grafting on CB via thermally generated radical polymers.

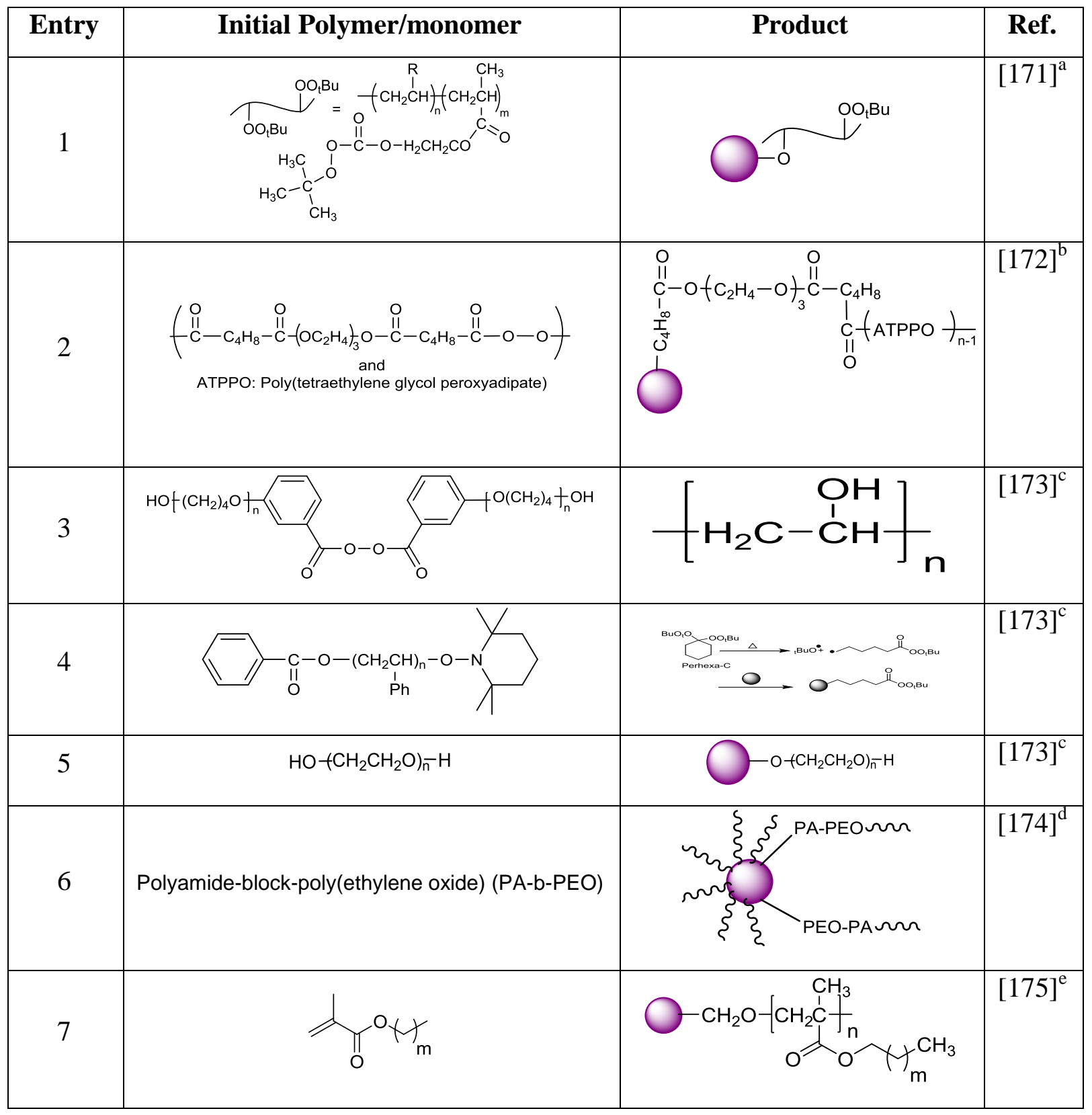




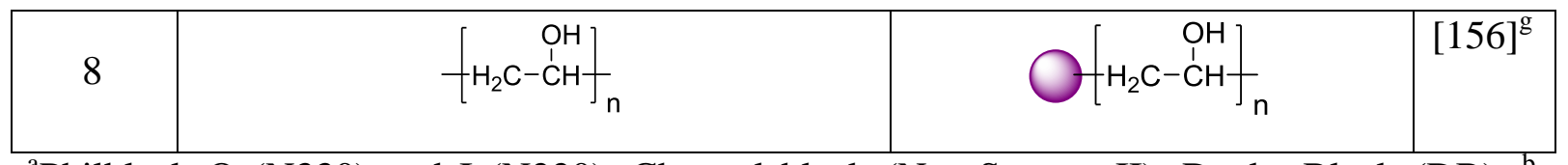

${ }^{a}$ Philblack O (N330) and I (N220), Channel black (Neo-Spectra II), Denka Black (DB). Philblack O (N330). ${ }^{\mathrm{c}}$ VXC $605 .{ }^{\mathrm{d}}$ Porousblack. ${ }^{\mathrm{e}} \mathrm{SB} 4 .{ }^{\mathrm{g}} \mathrm{CORAX}^{\circledR} \mathrm{N} 774$.

\subsection{Alternative approaches}

In 1972, Kinoshita and Bett first reported a method based on the electrochemical oxidation (corrosion) of CB (Neo-Spectra II) in concentrated phosphoric acid at a temperature of 135 ${ }^{\circ} \mathrm{C}$. The investigated parameters included the total anodic current, the amount of $\mathrm{CO}_{2}$ evolved and the oxygen content of the carbon as a function of time and potential. They also discussed a few mechanisms defining the electrochemical corrosion processes of $\mathrm{CB}$ by which both oxide growth and $\mathrm{CO}_{2}$ formation occur concurrently but as two independent reactions, and with the $\mathrm{CO}_{2}$ evolution eventually becoming the main process [176]. This approach offers a tuneable level of oxidation up to $15 \%$ by adjusting potentials from 700 to $1000 \mathrm{mV}$, nevertheless, long processing time restricts its scalability.

Electrochemical oxidation of $\mathrm{CB}$ was observed at both high and low potentials. At high potentials carbon was directly oxidized in the presence of water to produce $\mathrm{CO}_{2}, \mathrm{HCOOH}$, and $\mathrm{CO}$ as by-products. Instead, a general mechanism of $\mathrm{CB}$ oxidation at low potential was suggested by Chaparro et al., as shown in Eq 1-3, consisting in the reduction of dissolved oxygen to hydrogen peroxide that then acted as the oxidation agent of CB [177].

$$
\begin{aligned}
& 2 \mathrm{O}_{2}+4 \mathrm{e}^{-}+4 \mathrm{H}^{+} \rightarrow 2 \mathrm{H}_{2} \mathrm{O}_{2} \\
& \mathrm{C}+\mathrm{H}_{2} \mathrm{O}_{2} \rightarrow \mathrm{CO}+\mathrm{H}_{2} \mathrm{O} \\
& \mathrm{CO}+\mathrm{H}_{2} \mathrm{O}_{2} \rightarrow \mathrm{CO}_{2}+\mathrm{H}_{2} \mathrm{O}
\end{aligned}
$$

Another alternative oxidation method is the oxygen plasma. This method has been found to more uniformly oxidize the $\mathrm{CB}$ surface, when instead the wet oxidation methods produce ununiform $\mathrm{CB}$ preferentially oxidized at the edges of the BSU and in the amorphous domains present on the surface. This includes the amorphous portion, and the edge and basal plane sites of the ordered BSUs, performed under milder conditions than required with other oxidation methods [178]. Oxygen plasma generation of oxygen-containing functional groups, such as carboxyl, hydroxyl, lactone, and carbonyl groups, on the CB (ASTM N220) surface has also been reported by Park et al. [179]. Besides, oxygen plasma efficiently introduces 
surface oxygen functionalities without adding impurities onto the $\mathrm{CB}$ surfaces, as demonstrated for ASTM N200. This process also minimizes carbon burn-off or significant changes in bulk properties [180]. Although it has been established as one of the cleanest methods in industry, the oxygen plasma treatment leads to OCBs with lower oxygen contents than those obtained from wet oxidation methods.

The mechanism oxidation of $\mathrm{CB}$ with plasma-generated atomic oxygen is not fully understood at present, but it is assumed that the BSU edges terminated with hydrogen are involved in the formation of reactive intermediates, such as hydrogen and carbon radicals formed during oxygen plasma treatment. These radical intermediates are stabilized through electronic delocalization in the graphene planes [150] leading to numerous possible structural rearrangements. Each rearrangement yields broadly differing functionalities on the $\mathrm{CB}$ surface upon oxidation $[113,179]$. Figure 35 schematically represents some of the possible reaction pathways for $\mathrm{CB}$ oxidation which are likely to involve reactive oxygen intermediates formed during oxygen plasma or other oxidation reactions, such as treatment with piranha or $\mathrm{H}_{2} \mathrm{O}_{2}$ solutions $[122,181]$. The mechanisms suggest either ozonolysis or participation of atomic oxygen species in cleaving $\mathrm{C}=\mathrm{C}$ bonds, followed by further oxidation and dehydration, thus forming a variety of oxygen functionalities. Importantly, the existence of the $\mathrm{OH}, \mathrm{COOH}$, five-membered ring like cyclic anhydride etc. has been proposed for either oxidized CB or activated carbon $[113,182]$ according to X-ray photoelectron spectroscopy and FTIR analysis. Atomic oxygen can also be inserted at the edges where a radical [183] or a hydrogen atom bound to an unsaturated carbon is available [132]. 


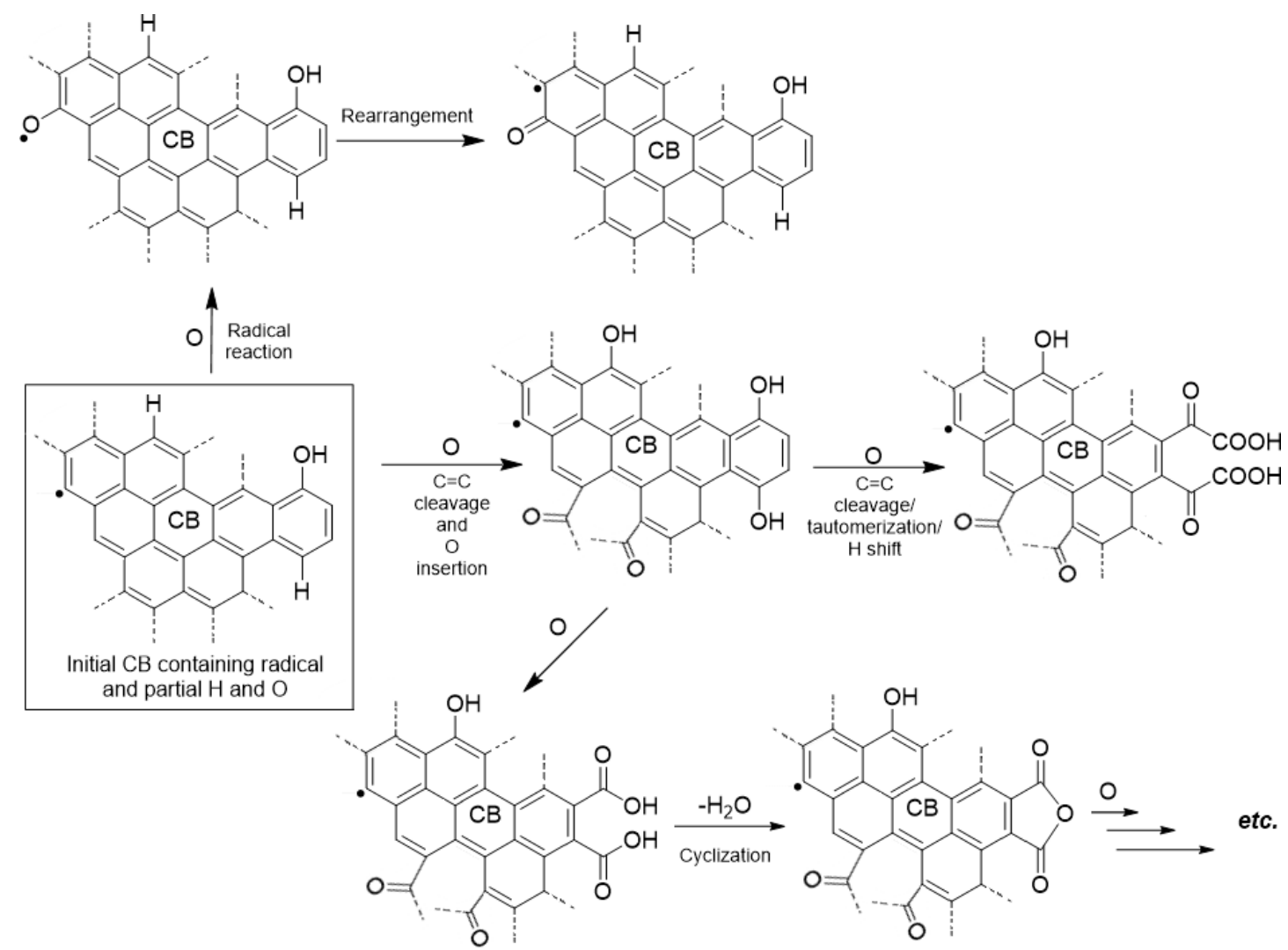

Figure 35 - Carbon surface functionalization involving reactions with atomic oxygen. Radical, hydrogen, and oxygen content is shown in the starting CB structure, as highlighted in the square frame. The dashed lines indicate the extension of the graphene plane. Partially adapted and expanded with permission from reference [122].

Oxygen and nitrogen functionalities have also been introduced in CB using mechanochemical approaches. Recently, ball milling mechanochemistry has attracted much attention due to its clean nature, high speed, and simple operation which can contribute to existing and new solid chemistry strategies. Mechanochemistry provide a simple means to tailor the porosity and improve the wettability of $\mathrm{CB}$. For instance, ball milling in the presence of ammonia solution produced nitrogen functionalized $\mathrm{CB}$ with increased surface area as compared to the starting material (Figure 36). The area increased from $67 \mathrm{~m}^{2} / \mathrm{g}$ to 307 $\mathrm{m}^{2} / \mathrm{g}$, and the total nitrogen and oxygen content increased to $2.5 \mathrm{wt} \%$ and $8.7 \mathrm{wt} \%$, respectively. Oxygen was introduced at the fractured sites, where covalent $\mathrm{C}-\mathrm{C}$ bonds were broken [184]. 


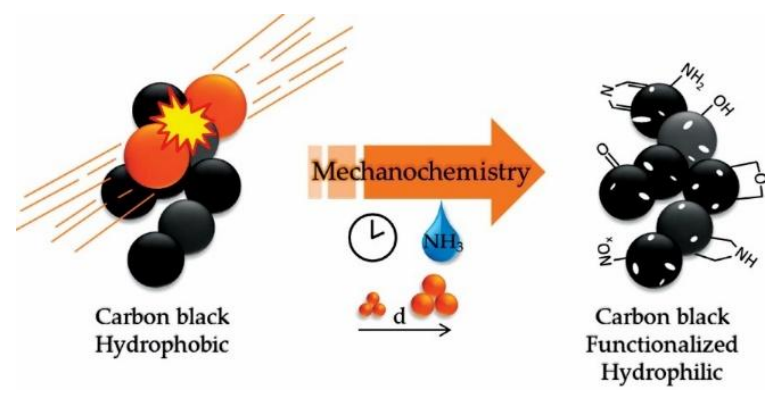

Figure 36 - Mechanochemical functionalization of $\mathrm{CB}$ in a ball mill. Reprinted with permission from reference [184]. Copyright 2009 MDPI.

\section{Emerging energy and environmental applications of CB-based materials}

\subsection{Fuel cells}

Fuel cells are electrochemical cells that convert the chemical energy stored in $\mathrm{H}_{2}$ or another fuel source into electricity through an electrochemical reaction with $\mathrm{O}_{2}$ or another oxidizing agent [185]. Many types of fuel cells exist, being these classified according to the electrolyte used. The most commonly investigated fuel cells are the proton exchange membrane fuel cell (PEMFC), direct methanol fuel cell (DMFC) or direct formic acid fuel cell (DFAFC), alkaline fuel cell (AFC), phosphoric acid fuel cell (PAFC), and solid oxide fuel cell (SOFC). Fuel cells consist of an anode, a cathode, and an electrolyte that allows protons or other charged species to move between the two sides of the fuel cell. In a typical PEMFC, a catalyst, i.e. $\mathrm{Pt}$, promotes the oxidation of $\mathrm{H}_{2}$ at the anode, thus generating protons and electrons. The protons flow from the anode to the cathode through the electrolyte after the reaction and the electrons flow from the anode to the cathode through an external circuit. The electrolyte is supported on an ionomer Nafion membrane. Nafion is a copolymer of sulfonated tetrafluoroethylene and perfluorinated vinyl ether, and it was first produced and commercialized by DuPont. Nafion is known for its excellent proton conductivity, thermal and mechanical stabilities, all desired properties for PEMFC application [186]. At the cathode, a catalyst promotes the oxygen reduction reaction (ORR) with the protons and electrons to form water. The net reaction yields $\mathrm{H}_{2} \mathrm{O}$, and the external current is used for power. The diffusion of gases in both anode and cathode sides of the cell is controlled by a gas diffusion layer (GDL) membrane, which must resist oxidation in order to minimize interaction with water and prevent side reactions. The assembled electrode containing a catalyst layer and the GDL is the gas diffusion electrode (GDE) [185, 187]. 
Due to the high cost of Pt and to the low specific surface area (SSA) and high density of the bulk metal, Pt is dispersed as finely divided nanoparticles onto an inexpensive, chemically and thermally stable, and electronically conductive support. The support also plays a role of maximizing access to the catalyst while stabilizing the Pt-NP and preventing their aggregation and sintering during fuel cell operation. Carbon materials in general, and quite extensively $\mathrm{CB}$, have been investigated as catalyst supports or as the catalyst material in fuel cell electrodes and GDL [11, 187-191]. Examples of CB commonly tested for fuel cells include Black Pearls (BP 2000), Ketjenblack® (KB EC600JD and EC300J), Shawinigan and Denka Black [192]. A key property of CB affecting performance is the surface area. For example, VULCAN XC-72R has been widely used as catalyst support due to its high SSA and good electrical conductivity, thus displaying better performance for PEMFC when compared to acetylene black, and in GDL than Shawinigan as cathode supports in PEMFC [187]. The SSA has been found to help control the catalyst NP size, as in higher SSA CB yielding smaller NP. This effect is due to increase in small mesopores due to CB particle aggregation, presence of oxygen surface functional groups, and micropores within the $\mathrm{CB}$ particles $[189,193]$.

The existence of these deep micropores and small mesopores, however, have detrimental effects for devices such as PEMFC, DMFC or DFAFC.[188, 192] The reason lies in the fact that Nafion, the proton conductive polymer used for assembling the membranes, forms micelles greater than $40 \mathrm{~nm}$ in size $[188,189,192]$. Therefore, the limited contact between the proton conducting membrane and the anode restricts the proton transfer to catalyst NP confined to pores smaller than $2 \mathrm{~nm}$. Therefore, electroactive surface areas may be drastically low, meaning, most NPs will not be participating in redox processes during fuel cell operation [188]. To avoid trapping of small Pt-NP into deep micropores, hexapod Pt-NP with roughly $20 \mathrm{~nm}$ in size and having an excess of (111) surfaces, presented in Figure 37, exhibit higher catalytic activity than commercial Pt/C catalysts [194]. Despite its bigger size, hexapods had high active surface area (ASA) of $62.4 \mathrm{~m}^{2} / \mathrm{g}$, compared to $2.7 \mathrm{~m}^{2} / \mathrm{g}$ of commercial Pt/C composites. The electroactivity of hexapod Pt-NP deposited on VULCAN $\mathrm{XC}-72 \mathrm{R}$ for $\mathrm{MeOH}$ oxidation in $\mathrm{HClO}_{4}$ reached $0.26 \mathrm{~mA} / \mathrm{cm}^{2}$ and $0.35 \mathrm{~V}$, versus 0.11 $\mathrm{mA} / \mathrm{cm}^{2}$ and $0.34 \mathrm{~V}$ of commercial Pt/C. An added benefit of working with excess (111) surfaces, was that less carbonaceous deposits formed on the surfaces of hexapods than onto commercial Pt/C. From a broader perspective, despite the large size of Pt-hexapods, small Pt NPs of $\sim 2.4 \mathrm{~nm}$ in size had $42.8 \mathrm{~m}^{2} / \mathrm{g}$ ASA. Reported mass activity for the latter Pt NPs on 
the same Vulcan XC-72R support was $313.3 \mathrm{~mA} / \mathrm{mg} \mathrm{Pt}$ and $0.732 \mathrm{~mA} / \mathrm{cm}^{2} \mathrm{Pt}$ area activity for $\mathrm{MeOH}$ oxidation. For reference, a commercial Pt/C had $37.2 \mathrm{~m}^{2} / \mathrm{g} \mathrm{ASA}, 264.6 \mathrm{~mA} / \mathrm{mg} \mathrm{Pt}$ and $37.2 \mathrm{~m}^{2} / \mathrm{g}$ mass and area activity, respectively.

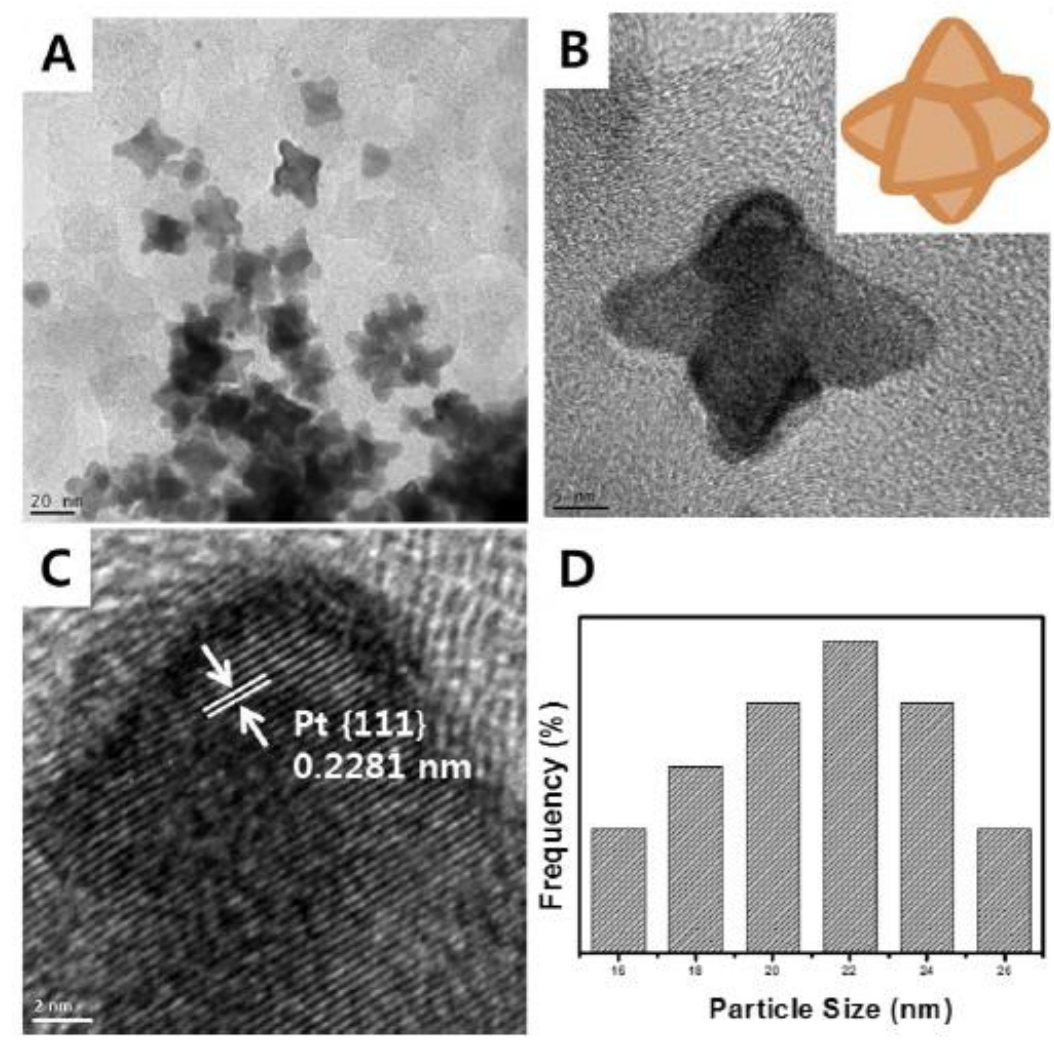

Figure 37 - TEM of Pt-hexapod NPs on commercial CB (A); HRTEM image of Pt-hexapod NP (B) and of (111) Pt plane (C); representative particle size distribution of Pt-hexapod NPs (D). Adapted from reference [194].

In general, $\mathrm{CB}$ suffer from poor chemical stability in highly acidic and in highly alkaline conditions. Very low, or very high $\mathrm{pH}$ leads to corrosion of $\mathrm{CB}$ electrodes and catalyst NP to detach from support surfaces. $\mathrm{CB}$ are also susceptible to oxidation at low temperatures at potentials greater than $1.2 \mathrm{~V}$ versus the standard hydrogen electrode (SHE), and at high temperatures at potentials approaching $0.9 \mathrm{~V}$ versus SHE. Oxidation often results in loss of ASA, changes in pore structure and in surface functional groups [188]. CB have surface oxygen functional groups, most of which are acidic, and the stability of these groups further affects the stability of the supported NP catalysts [189]. For Pt-NP, the precursor used is the acid $\mathrm{H}_{2} \mathrm{Pt}(\mathrm{Cl})_{6}$, and basic surface sites are preferred for strong interaction and better dispersion of final NPs. Therefore, oxidation methods that lead to increase in surface acidity of carbons, do not improve NP dispersion, and the solution $\mathrm{pH}$ for Pt impregnation is also 
important. Furthermore, due to the different SSA and oxygen content, CB display different interactions with Pt. For instance, REGAL 660R has been found to stabilize Pt-NP against sintering, whereas BP 2000 has lower corrosion resistance than VULCAN XC-72R [189]. A way to improve the interaction of Pt with the CB surfaces, is the alloying of $\mathrm{Pt}$ with $\mathrm{Ru}$, which binds more strongly to carbon surfaces and without greatly impacting the ASA of the electrocatalysts $[11,187]$. By employing Pt-Ru NP, some authors have reported that preoxidation of $\mathrm{CB}$ surface with hydrogen peroxide $\left(\mathrm{H}_{2} \mathrm{O}_{2}\right)$ resulted in better ORR activity of the catalysts and to better overall NP stability in PEMFC and DMFC due to better NP dispersion and accessibility. For instance, the SSA of $\mathrm{H}_{2} \mathrm{O}_{2}$ treated VULCAN XC-72R was reduced from $241 \mathrm{~m}^{2} / \mathrm{g}$ to $166 \mathrm{~m}^{2} / \mathrm{g}$ [195]. This decrease in SSA decreased NP access to deep micropores, which favored contact with Nafion membrane in the cells.

Other systems of interest include cells using alternative hydrogen sources. These sources include ammonia [196] and hydrazine [197]. One of the advantages of using ammonia or hydrazine, for example, is that it generates more power than $\mathrm{H}_{2}$ fuel cell and without Ptcatalyst. Similar to $\mathrm{H}_{2}$ fuel cells, hydrazine fuel cells have also shown positive effects linking CB chemical treatment to increased catalytic activity of the supported metal NPs. For example, the activity of silver on oxidizing acid pretreated CB was found to increase for the hydrazine oxidation [11]. Acetylene black treated in $\mathrm{KOH}$ in an anodic oxidation cell, made this CB more hydrophilic, and with lower electrical conductivity. Surface areas also decreased from $64 \mathrm{~m}^{2} / \mathrm{g}$ down to $47 \mathrm{~m}^{2} / \mathrm{g}$. The pore volumes of these OCBs also decreased slightly from $1.13 \mathrm{~cm}^{3} / \mathrm{g}$ to as low as $0.85 \mathrm{~cm}^{3} / \mathrm{g}$. Such changes improved the $\mathrm{H}_{2} \mathrm{O}_{2}$ decomposition performance, with optimum SSA of $52 \mathrm{~m}^{2} / \mathrm{g}$ being reported for this reaction [198]. Likewise, electrochemical pre-treatment of C impacts the Au-NP activity for ORR onto the carbons support [199]. The peak potential for this reaction was directly linked to the applied pre-treatment potential. Oxidation of CB with SSAs of $\sim 62 \mathrm{~m}^{2} / \mathrm{g}$ and $50 \mathrm{~nm}$ particle size (c-nergy super C65) in piranha solution (3:1 V/V $\left.\mathrm{H}_{2} \mathrm{SO}_{4}: \mathrm{HNO}_{3}\right)$, or electrochemically in $\mathrm{KOH}$ afforded metal-free catalysts for the water oxidation reaction (WOR) or alcohol oxidation reaction (AOR) (i.e. methanol, ethanol) [138]. WOR is strongly dependent on applied potential when using $\mathrm{OCB}$, as additional carbon oxidation takes place above $1.70 \mathrm{~V}$ and $1.80 \mathrm{~V}$. At this stage, faradaic efficiency dropped from $95 \%(1.59 \mathrm{~V}$, and current density of $0.65 \mathrm{~mA} / \mathrm{cm}^{2}$ ) down to $32 \%$ and $18 \%$, respectively. Electrochemical oxidation of these same $\mathrm{CB}$ in $\mathrm{KOH}$ proved even more efficient than piranha solution. For both WOR and AOR processes, ketone surface groups are responsible for the catalytic activity, and the 
electrochemical process yielded more of these groups, in addition to some degree of $\mathrm{CB}$ exfoliation during $\mathrm{KOH}$ intercalation and $\mathrm{CB}$ particle growth [138]. Other authors have reported on the surface oxidation of VULCAN XC-72R using $\mathrm{MeOH} / \mathrm{H}_{2} \mathrm{SO}_{4}, \mathrm{O}_{3}$ improved dispersion of Pt-Ru NP, and consequently catalyst activity, where $\mathrm{O}_{3}$ oxidation of a generic furnace black sample was found to yield surface basic lactone and phenol groups [200]. Whereas, citric acid treatment of VULCAN XC-72R resulted in smaller Pt particles on CB, rich in carboxyl and hydroxy groups [189, 201].

A drawback of surface oxidation is the strong interaction with water in fuel cells, as in the GDL [187, 189, 190]. For all devices, surface hydrophobicity is desired for fast water removal and reduced oxidation of the device and etching of catalyst NPs. For instance, CB particles treated at temperatures used for graphitization are preferred supports for Pt-alloys cathode catalyst used for ORR. The graphitization process makes the CB surfaces more hydrophobic, which is important for fast $\mathrm{H}_{2} \mathrm{O}$ removal, and that in turns makes the catalyst less prone to corrosion [190]. Graphitized CB-based anodes have also been found to increase the tolerance to carbon monoxide (CO) of Pt-Ru NPs supported on CBs in PEMFCs [190]. As discussed in previous sections, graphitization leads to improved ordering of graphene sublattice, BSU growth and loss of functional groups from heteroatoms. Such defects and oxygen functionalities reduce electrical conductivity of carbons. Not surprisingly, fluorinating $\mathrm{CB}$ surfaces with $\mathrm{C}_{2} \mathrm{~F}_{2}$ leads to increased surface hydrophobicity but lowers electrical conductivity due to formation of $\mathrm{C}-\mathrm{F}$ bonds and disruption of the delocalized $\mathrm{sp}^{2}$ carbon lattice [202, 203]. The fluorinated CB materials, however, were still good catalyst supports for PEMFC [189]. Other surface treatments, include low temperature thermal treatments under air or $\mathrm{N}_{2}\left(\sim 350^{\circ} \mathrm{C}\right)$, which has been found to improve the stability of CBs in $\mathrm{H}_{2} \mathrm{SO}_{4}$ by cleaning the $\mathrm{CB}$ surfaces [189]. Also, physical activation at $800-1100{ }^{\circ} \mathrm{C}$ under inert atmospheres or using air-steam have been reported to remove surface oxygen groups off VULCAN XC-72R and Shawinigan surfaces, while simultaneously increasing its surface areas by introducing micropores. Pt-supported on these activated CBs had higher ASAs than Pt-supported on nonactivated CBs or even over fullerene soot [189].

Alternatively, CB composites have been found to solve some of the issues associated with conductivity losses, catalyst dispersion and stability, or formation of deep micropores. Hydrophobicity and porosity in GDL cathodes in PEMFC have been balanced by mixing different types of CB particles forming the membrane [204]. For instance, upon mixing acetylene black (SSA $\sim 62 \mathrm{~m}^{2} / \mathrm{g}$ ) with BP 2000, membranes with tailorable thickness, and 
SSA of $335 \mathrm{~m}^{2} / \mathrm{g}$ could be assembled. While electrical conductivity was comparable for composites and individual CB-type membranes, composites had higher permeability than BP 2000 and higher hydrophobic porosity than acetylene black. The latter properties could be tailored by adjusting the acetylene black to BP 2000 ratios, thus favoring water removal and decreased mass polarization losses. Peak performance reached $0.91 \mathrm{~W} / \mathrm{cm}^{2}$ for $10 \mathrm{wt} \% \mathrm{BP}$ 2000 loading in membranes. Another benefit of mixing was the avoidance of micron-sized cracks on the surfaces of composite membranes, which were only observed in acetylene black, or in BP 2000 membranes. Similarly, membranes prepared using acetylene black, P702 and P701 CBs, were investigated for $\mathrm{O}_{2}$ electroreduction into a $\mathrm{H}_{2} \mathrm{O}_{2}$ solution [205]. The optimum membrane thickness with $70 \%$ open porosity was found to be in the range of 0.2-0.3 mm for the working layer, yielding 24-29 g/ $\mathrm{H}_{2} \mathrm{O}_{2}$ from atmospheric $\mathrm{O}_{2}$. CB-reduced graphene oxide ( $\mathrm{GGO}$ ) composites have also been investigated for ORR, by mixing $\mathrm{CB}$ with graphene oxide (GO), followed by reduction [206]. Composites were found to stabilize PtNPs and promote stability, with $95 \%$ activity being retained in $\mathrm{O}_{2}$ saturated $\mathrm{HClO}_{4}$ solution. While $\mathrm{CB}$ was found to provide the active sites to capture and re-nucleate dissolved Pt, rGO prevented leaching of Pt into the electrolyte solution.

CB-polymer composites have also been investigated in order to promote NP dispersion and accessibility. VULCAN XC-72R composites with PEI were investigated as Pt support in PEMFC [207]. PEI loading affected Pt size, with smallest NPs and narrower size distributions obtained at $0.4 \mathrm{wt} . \%$ PEI in composite support. At this optimum composition, the highest power was obtained in $\mathrm{H}_{2} \mathrm{SO}_{4}$ medium, of $1.05 \mathrm{~W} / \mathrm{cm}^{2}$ at $2.31 \mathrm{~A} / \mathrm{cm}^{2}$. Pt-supported CB-PEI had similar ASA to commercial Pt/C. CB-PEI composites, however, were much stable, retaining nearly $50 \%$ of ASA after 1200 cycles versus less than $30 \%$ retention for the reference $\mathrm{Pt} / \mathrm{C}$.

Similarly, Pt-Pd NPs deposited onto VULCAN XC-72R-PEI composites displayed enhanced activity over Pt-Pd NPs on solely VULCAN XC-72R for MeOH oxidation in $\mathrm{H}_{2} \mathrm{SO}_{4}$ [208]. Results indicate that besides favoring better NP dispersion onto supports, PEI is also an electrical conductor. ASA for Pt-Pd on PEI-CB composites reached $68 \mathrm{~m}^{2} / \mathrm{g}$, with $\mathrm{Pt}-\mathrm{Pd}$ crystallite size of $1.9 \mathrm{~nm}$. For the CB supported catalysts, Pt-Pd had ASA of $55 \mathrm{~m}^{2} / \mathrm{g}$, and crystallites of $2.4 \mathrm{~nm}$ in size. PEI further improved catalyst stability and tolerance to carbon monoxide (CO) [208]. Future advances in the preparation of low-cost, CB-conductive polymer composites with high electrical conductivity and permeability for GDL, and DFAFC. For the latter, recent studies showed that Pd-supported on conducting titanium 
suboxide (Magnéli phase) $\mathrm{Ti}_{4} \mathrm{O}_{7}$ exhibited higher ASA $\left(47.2 \mathrm{~m}^{2} / \mathrm{g}\right.$ ) for FA oxidation than Pd on VULCAN XC-72R $\left(22.1 \mathrm{~m}^{2} / \mathrm{g}\right)$ [209]. Alloying Gd with Pd further shift the CO oxidation potentials in $\mathrm{H}_{2} \mathrm{SO}_{4}$ from $-0.55 \mathrm{~V}$ to $-0.45 \mathrm{~V}$, where $\mathrm{Ti}_{4} \mathrm{O}_{7}$ improved performance at high current densities and favored reduction of Pd. The abundance of conducting polymers and of CBs, however, could lead to further improvements in oxidation potentials, catalyst stability and tolerance to $\mathrm{CO}$, at lower cost, and lighter weight over inorganic oxide supports.

The alloying of Pd with boron (B) has also been found to improve stability of the NP catalysts on VULCAN XC-72R in DFAFC [210]. Pd-B NP prepared using borane dimethylamine, $\left(\mathrm{CH}_{3}\right)_{2} \mathrm{NH} \cdot \mathrm{BH}_{3}$, were $4 \mathrm{~nm}$ in size, and exhibited improved long-term stability over NP prepared with sodium borohydride, $\mathrm{NaBH}_{4}$, especially after thermal annealing. Although B may not be catalytically active in the Pd-B alloys, metal-free B, Ndoped CBs have been found active catalysts for ORR [211]. These materials were prepared by high-energy ball-milling of hexagonal boron nitride (h-BN) with $\mathrm{CB}$ particles. This mechanochemical alloying process resulted in $\mathrm{B}-\mathrm{C}$ and $\mathrm{C}-\mathrm{N}$ bond formation, with simultaneous increase in the $\mathrm{d}_{002}$-spacing of h-BN lattice and loss of its graphitic structure. The B, N-doping levels studied ranged from $0 \mathrm{wt} . \%$ to $20 \mathrm{wt} . \%$, and although activity for ORR in $\mathrm{O}_{2}$-saturated $\mathrm{H}_{2} \mathrm{SO}_{4}$ electrolyte was below that of commercial $\mathrm{Pt} / \mathrm{C}$, further improvements in alloying process are expected to lead to metal-free catalysts with activity approaching that of Pt/C. Previous studies have focused on the role of N-doping in carbons, as in the $\mathrm{NH}_{3}$ activation of furnace black at $950^{\circ} \mathrm{C}[212,213]$. The SSA of activated carbons exceeded $800 \mathrm{~m}^{2} / \mathrm{g}$, with micropores forming from the burn off of disordered carbon in the CB particles. Iron acetate was further added to furnace black during activation study, and this work developed a model to predict ORR activity of these materials [212]. Other studies have suggested that N-doping allowed for the control of Fe NP catalysts, and in final activity, whereas $2 \mathrm{wt} . \%$ to $8 \mathrm{wt} . \% \mathrm{~N}$-doping in $\mathrm{CB}$ from the pyrolysis of urea-formaldehyde resins, was also active in the reduction of $\mathrm{O}_{2}$ into $\mathrm{H}_{2} \mathrm{O}_{2}$. A more detailed study was reported for $\mathrm{N}$ doped Ketjenblack® EC 300J materials prepared using formaldehyde cross-linked resins with melamine, urea, thiourea, and selenourea [133]. The CB were first oxidized in $\mathrm{HNO}_{3}$ prior to mixing with resins and pyrolysis between $400^{\circ} \mathrm{C}$ and $1000^{\circ} \mathrm{C}$ under $\mathrm{N}_{2}$ atmosphere. $\mathrm{N}$-doped Ketjenblack ${ }^{\circledR}$ particles had varying ratios of N, more specifically of graphitic Nsites to quaternary $\mathrm{N}$-sites in the carbon lattice. Optimum catalysts formulations were obtained from selenourea precursors pyrolyzed at $800^{\circ} \mathrm{C}$, for which the onset potential for ORR was of $0.76 \mathrm{~V}$ (SHE), and the amount of $\mathrm{H}_{2} \mathrm{O}_{2}$ produced at $0.5 \mathrm{~V}$ (SHE) reached $\sim 3 \%$ in 
$\mathrm{O}_{2}$ saturated $\mathrm{H}_{2} \mathrm{SO}_{4}$. Results indicated synergistic effects of lone-electron pair in quaternary $\mathrm{N}$-sites and additional electron donated by graphitic $\mathrm{N}$-site to the delocalized $\pi$-system in the BSU for the facilitated reduction of $\mathrm{O}_{2}$. It was further highlighted that $\mathrm{HNO}_{3}$ oxidation introduced quinone groups, which enhanced performance of $\mathrm{N}$-doped $\mathrm{CB}$ due to higher double-layer capacitance and consequently higher onset potentials for ORR [133]. Preoxidation of acetylene black by a modified Hummers method using sodium nitrate $\left(\mathrm{NaNO}_{3} \cdot \mathrm{H}_{2} \mathrm{O}\right)$ in $\mathrm{H}_{2} \mathrm{SO}_{4}$ was found to favor $\mathrm{N}$-doping, $\sim 6.3 \mathrm{wt} . \% \mathrm{~N}$, during $\mathrm{NH}_{3}$ activation in the presence of manganese chloride $\left(\mathrm{MnCl}_{2}\right)$ as illustrated in Figure 38 [214]. The SSAs increased from $77 \mathrm{~m}^{2} / \mathrm{g}$ for acetylene black to $\sim 140 \mathrm{~m}^{2} / \mathrm{g}$ for the Mn-free, N-doped CB. Final materials had good ORR activity in $0.1 \mathrm{M} \mathrm{KOH}$ with onset potential of $1.0 \mathrm{~V}$ (SHE), in a onestep 3.92 electron process, due to prevailing pyridinic and pyrrolic $\mathrm{C}-\mathrm{N}$ sites.

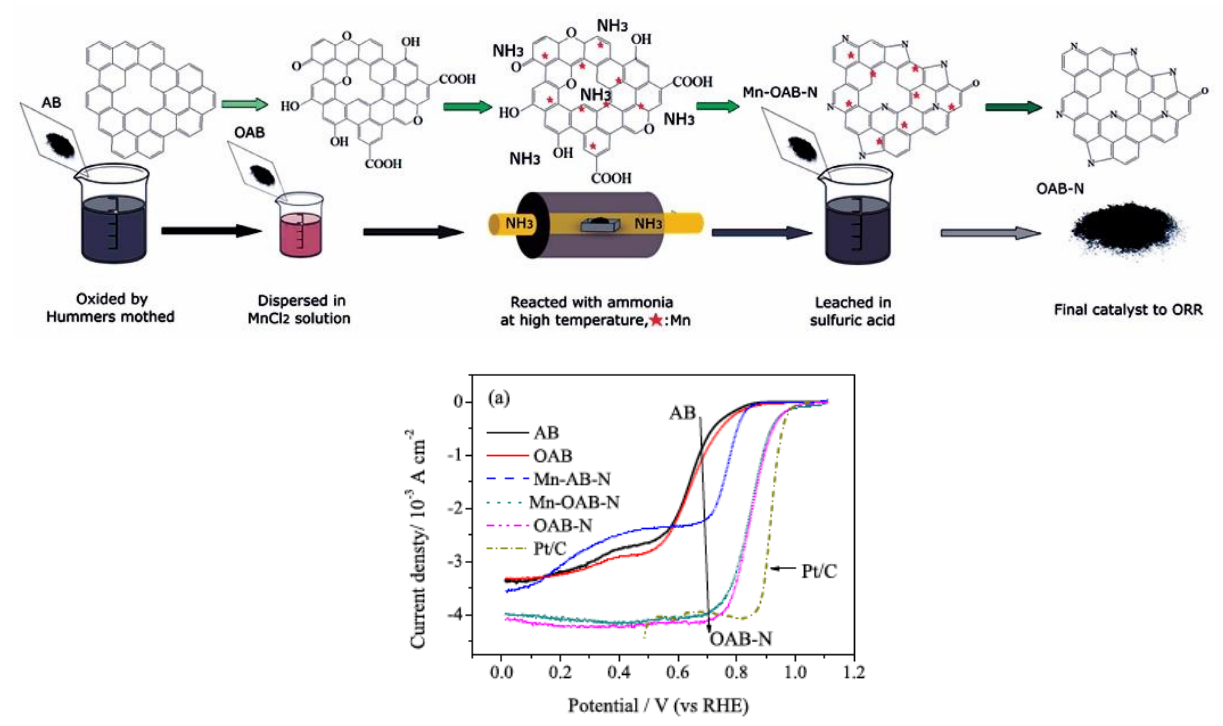

Figure 38 - Scheme illustrating the preparation of $\mathrm{N}$-doped acetylene black using $\mathrm{MnCl}_{2}$ oxidation catalyst solution (left) and LSV curves of acetylene black (AB), oxidized acetylene black (OAB), reduced Mn-containing N-doped acetylene black (AB-N), oxidized Mn-OAB$\mathrm{N}$, final metal-free OAB-N and commercial $20 \mathrm{wt} . \% \mathrm{Pt} / \mathrm{C}$ catalyst obtained at a rotation speed of $900 \mathrm{rpm}$ in $0.1 \mathrm{M} \mathrm{KOH}$, with 10mV/s. Adapted from reference [214].

Recent studies have demonstrated that $\mathrm{N}$-doped $\mathrm{CB}$ exhibit better activity for ORR in alkaline electrolytes than commercial $\mathrm{Pt} / \mathrm{C}$. These $\mathrm{N}$-doped $\mathrm{CB}$ were prepared by the pyrolysis of poly-p-phenylenediamine onto BP 2000 [215]. Electrooxidation on N-doped CB followed a 4-electron process, with higher current density and more positive half-wave potential than $\mathrm{Pt} / \mathrm{C}$, i.e. $2.17 \mathrm{~mA} / \mathrm{cm}^{2}$ vs $1.11 \mathrm{~mA} / \mathrm{cm}^{2}$ at $0.1 \mathrm{~V}$. Durability tests revealed decreased activity over time due to contamination of catalytically active sites from exposure 
to oxygen, as evidenced by increased O-contents in N-CB. Furthermore, ORR activity decreased with increasing current densities to $0.29 \mathrm{~mA} / \mathrm{cm}^{2}$ at $-0.1 \mathrm{~V}$ due to poor catalyst stability in air. N, F, and N-F-doped VULCAN XC-72R have also been tested for ORR in neutral pH media [216]. N, F-doped CB had the highest onset potential (-0.17V) over Fdoped CB (-0.20V). F-doping favors the coexistence of 2-electron and 4-electron processes, whereas co-doping with $\mathrm{F}$ and $\mathrm{N}$ favored a quasi-4-electron ORR process. Both F-CB and N, F-CB exhibited high SSAs, and enhanced tolerance for $\mathrm{MeOH}$ and $\mathrm{Na}_{2} \mathrm{~S}$ and good long-term electrochemical stability compared to $\mathrm{Pt} / \mathrm{C}$ at neutral $\mathrm{pH}$ electrolyte. Similar results have been reported for $\mathrm{N}$ and F-doped BP 2000 for ORR in alkaline conditions. F, N-CBs exhibited the best cathode performance for DMFC. BP 2000 had the lowest onset potential of $-0.22 \mathrm{~V}$ for 2-step 2-electron process. For either N-doped or F-doped CB, onset potential was $-0.15 \mathrm{~V}$ for a 1 -step 4 -electron process. For F, N-CB, the potential increased to $-0.04 \mathrm{~V}$ for this 1-step 4-electron reaction. Peak potential and half-wave potential for F, N-CB were $-0.20 \mathrm{~V}$ and $-0.12 \mathrm{~V}$, respectively. Results were comparable to commercial Pt/C. The activity of doped materials was attributed to high contents of pyrrolic and pyridinic $\mathrm{N}$-sites, and to ionic and to semi-ionic C-F bonds in or near lattice vacancy sites.

Despite the low costs of preparing metal-free catalysts, acidic electrolytes are preferred due to cell and electrode corrosion in alkaline $\mathrm{pH}$, and undesired alkali depletion upon reaction with atmospheric $\mathrm{CO}_{2}$. Such issues are not found for metal-based catalysts. As for metalbased electrooxidation, the effect of doping the carbon supports has been investigated. For instance, Pt-supported on N-doped CB have been prepared by plasma treatment of VULCAN XC-72R with allylamine, and $\mathrm{N}_{2}$; Pt-NP catalysts were then deposited onto these supports [217]. SSA of plasma treated CBs decreased from $256 \mathrm{~m}^{2} / \mathrm{g}$ to $212 \mathrm{~m}^{2} / \mathrm{g}$ for $\mathrm{N}_{2}$, and down to $152 \mathrm{~m}^{2} / \mathrm{g}$ for allylamine due to $\mathrm{CB}$ micropore blockage. Although $\mathrm{N}$-site information was not determined, N-doping introduced more basic surface sites, while lowering the numbers of carbon surface acid sites. This effect leads to better Pt-NP dispersion, size control, and Pt ASA. While NP were $\sim 2.46 \mathrm{~nm}$ in size for $\mathrm{N}_{2}$-plasma treated materials with $0.417 \mathrm{~cm}^{2} / \mathrm{g}$ ASA, these were $2.25 \mathrm{~nm}$ in size for allylamine-treated CB and ASA of $0.315 \mathrm{~cm}^{2} / \mathrm{g}$, versus $3.12 \mathrm{~nm}$ and $0.299 \mathrm{~cm}^{2} / \mathrm{g}$ on untreated VULCAN XC-72R. Pd-NP have also been deposited on 2dimensional hexagonal carbon nitride $\left(\mathrm{g}-\mathrm{C}_{3} \mathrm{~N}_{4}\right) / \mathrm{CB}$ nanocomposites [218]. The $\mathrm{g}_{-} \mathrm{C}_{3} \mathrm{~N}_{4}$ were obtained by oxidizing VULCAN XC-72R with $\mathrm{HNO}_{3}$, which was then mixed with melamine solution in ethylene glycol, followed by pyrolysis in $\mathrm{N}_{2}$ atmosphere at $350^{\circ} \mathrm{C}$ or $550^{\circ} \mathrm{C}$. Graphitic, tertiary, and primary N-sites prevailed in these nanocomposites, whereas N/C 
ratios were controlled by the melamine to $\mathrm{CB}$ ratios and pyrolysis temperature. As depicted in Figure 39, deposited Pd-NPs had broad size distributions over untreated VULCAN XC$72 \mathrm{R}$, whereas for $\mathrm{g}-\mathrm{C}_{3} \mathrm{~N}_{4}$ nanocomposites these were narrower, centered around $5 \mathrm{~nm}$ and were well-dispersed onto supports. Electrooxidation currents reached $2155 \mathrm{~mA} / \mathrm{mg} \mathrm{Pd}$ and $1720 \mathrm{~mA} / \mathrm{mg} \mathrm{Pd}$ in DFAFC and DMFC, respectively. Although the lowest charge transfer resistance was found for $\mathrm{Pd}-\mathrm{CB}$, also a consequence of the larger size of metallic NP and lack of semiconducting $\mathrm{g}-\mathrm{C}_{3} \mathrm{~N}_{4}$, the $\mathrm{g}_{-} \mathrm{C}_{3} \mathrm{~N}_{4}$ was found to protect the $\mathrm{CB}$ from corrosion in acidic and alkaline conditions. The $\mathrm{g}-\mathrm{C}_{3} \mathrm{~N}_{4}$ also promoted the stronger interaction of $\mathrm{Pd}$ with the support and their improved stability. Consequently, nanocomposites were able to retain their SSA and reactivity during cycling.
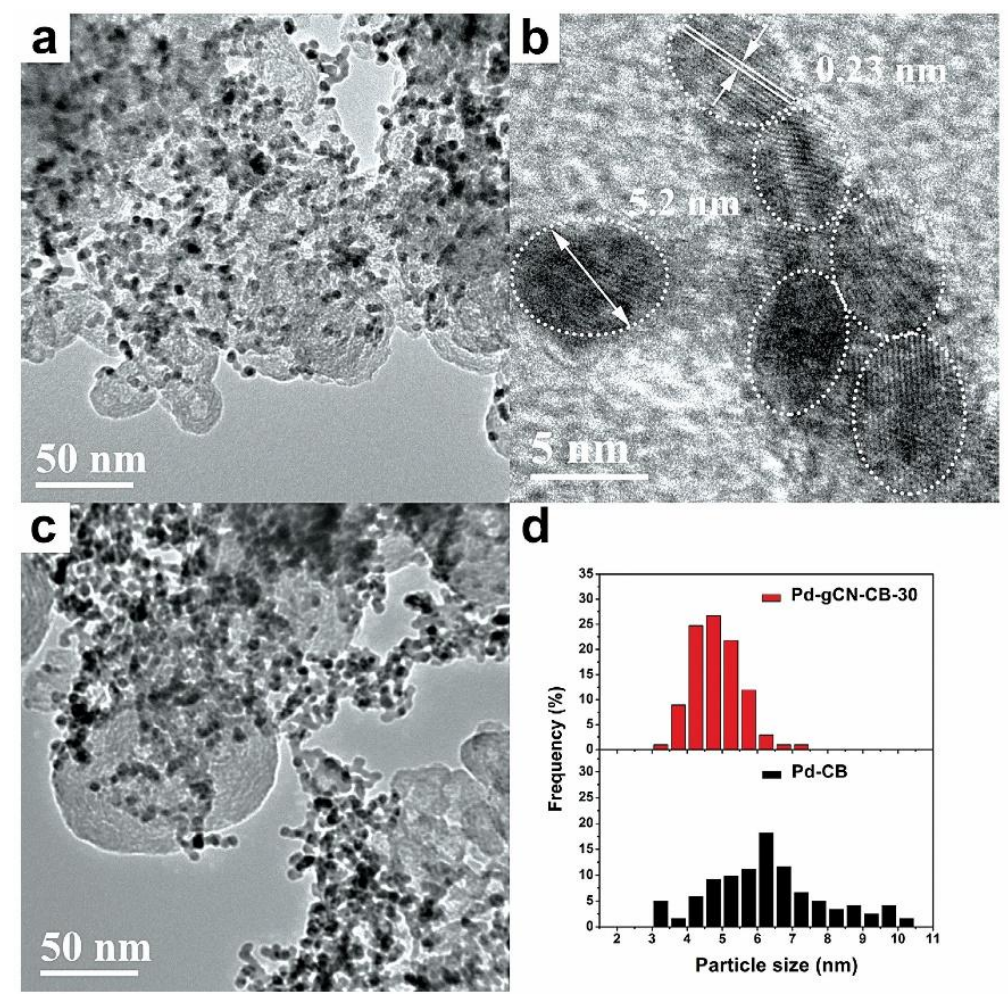

Figure 39 - Representative TEM (a) and HRTEM (b) images of Pd-g- $\mathrm{C}_{3} \mathrm{~N}_{4}-\mathrm{CB}$ with 30 wt.\% $\mathrm{CB}$; TEM image of Pd-CB (c) composite; particle size histograms of Pd-g- $\mathrm{C}_{3} \mathrm{~N}_{4}-\mathrm{CB}-30$ and Pd-CB materials. Adapted from reference [218].

While catalyst stability and costs are of major concern, one important recent development was that of graphene-CB composite cathodes for $\mathrm{O}_{2}$ reduction in GDE for the degradation of bisphenol A as a model pollutant in water [219]. By doping VULCAN XC-72R with 3wt.\% graphene, composites reduced $\mathrm{O}_{2}$ to $\mathrm{H}_{2} \mathrm{O}_{2}$ and then to decompose the peroxide into $\mathrm{OH}$ radical. In these, $\mathrm{CB}$ was found to be catalytically active in the reduction reaction into $\mathrm{H}_{2} \mathrm{O}_{2}$, 
and graphene provided the $\pi$-electrons for the peroxide decomposition in near neutral conditions. The membrane electrodes had large mesopores, which were nearly identical to that of undoped $\mathrm{CB}$, approximately 40nm in width. Despite the low levels of doping, the doping effect was clear; while 1wt.\% graphene was detrimental to the electrode performance, 3 wt.\% graphene largely increased the $\mathrm{H}_{2} \mathrm{O}_{2}$ and $\mathrm{OH}$ radical yield as compared to VULCAN XC-72R [219]. Higher doping levels of graphene have not been tested. The latter is of particular importance, due to the protective action of single-layer graphene to the VULCAN $\mathrm{XC}-72 \mathrm{R}$ layer in the GDE, especially for fuel cells operating at very alkaline $\mathrm{pH}$. Besides being hydrophobic and a good electrical conductor, both desirable properties for GDE, graphene has the potential to further improve the long-term stability of heteroatom doped-CB catalysts in ORR.

\subsection{Batteries}

Batteries are devices formed by one or multiple galvanic cells that store and deliver electrical energy through electrochemical reactions [220]. Battery components include the electrolyte(s), and a positive and a negative terminal that are physically separated by a membrane. Both terminals are connected through an external circuit through which electrons flow during the redox process. By convention, when a battery is discharging, the positive terminal is the cathode, where a positively charged species is reduced, and the negative terminal is the anode, where a species is oxidized. Thus, the anode is the source of electrons that flow through the external circuit. Different than fuel cells, batteries contain all of its components and do not require an external fuel source. Many different batteries exist, and these are classified according to the number of charge-discharge cycles. For instance, primary batteries (or cells) are single-use, whereas secondary batteries can be recharged and discharged numerous times [220]. Example of primary cells includes the alkaline batteries, and of secondary cells include $\mathrm{Ni}-\mathrm{Cd}, \mathrm{Ni}-\mathrm{Zn}$, nickel metal-hydride (Ni-MH), and lithium ion battery (LIB or Li-ion) cells. Recent research has also looked into inexpensive cations such as sodium (Na), and for multivalent cations that could lead to higher energy through increasing the number of electrons, e.g. magnesium $(\mathrm{Mg})$ and even having multiple possible oxidation states as iron $(\mathrm{Fe})[221,222]$. Also, of recent interest is the Zinc-Air battery, where $\mathrm{Zn}$ is oxidized by $\mathrm{O}_{2}$ from the air. This cell has high energy density and is of interest to power future electric vehicles (EV). It represents a hybrid system between battery and fuel cell. The latter technologies still require many more years of research until these can reach 
consumer products, and until that happens, most portable electronic devices and EV continue to move towards LIB [223].

In commercial LIB, the anode material is graphite, and a Li salt is dissolved in an organic solvent, usually a mixture of ethylene carbonate and diethyl carbonate. The organic solvents decompose on the surface of the graphite, forming a polycarbonate film on its surfaces, known as the solid electrolyte interphase (SEI). Li-cations lose their solvation shell when migrating to the anode through the SEI, then forming an intercalation compound with graphite that has an ideal stoichiometric composition of $\mathrm{LiC}_{6}$. Lithium is not reduced in the graphite, but rather interacts through charge-transfer with graphite. When a lithium metal anode is used as $\mathrm{Li}^{+}$source, the battery is classified as a half-cell. These batteries have very high energy densities but are unsafe and dangerous. Thus, graphite allowed for widespread commercialization of safer LIB. On the cathode side, oxides of aluminum, cobalt, nickel, manganese, vanadium, and iron phosphates, that have either layered or the spinel structure are used. The latter form intercalation compounds with $\mathrm{Li}^{+}$. For example, while $\mathrm{LiNiMnCoO}_{2}$ (NMC) is the most common cathode used in $\mathrm{EVs}, \mathrm{LiNiCoAlO}_{2}(\mathrm{NCA})$ is used by Panasonic in the battery cells powering the best-selling EVs in the United States, namely Tesla Models S, X, and 3 [224]. In this segment, the selected cathode material has been one of the important factors helping Tesla's EVs achieve the best overall performance, as top speed and mileage. Modern LIB with graphite anode thus have variable nominal potential depending on the cathode material selected [225]. Since these oxides have poor electrical conductivity, an additive is required to promote the conductivity of the cathode.

In this direction, $\mathrm{CB}$ has been extensively investigated as a major conductive component in composite battery cathodes. One of the first examples is that of $\mathrm{CB}-\mathrm{MnO}_{2}$ composites prepared by mixing $\mathrm{KMnO}_{3}$ with $\mathrm{AB}$ [226]. The system was then thermally treated, and the reaction between $\mathrm{KMnO}_{3}$ and carbon from $\mathrm{CB}$ surfaces yielded $\mathrm{MnO}_{2}$. The incomplete reaction left smaller CB particles in the oxide, which increased the electrical conductivity of the final composites. And the morphology of the $\mathrm{CB}-\mathrm{MnO}_{2}$ resembled that of the starting $\mathrm{CB}$ particle aggregates. When tested as cathode material for LIB, discharge capacities of 231 $\mathrm{mAh} / \mathrm{g}$ and $40 \mathrm{~mA} / \mathrm{g}$ versus Li were obtained. Another interesting development for LIBs, was that of $\mathrm{CB}$ nanocomposite cathodes with $\mathrm{LiMn}_{2} \mathrm{O}_{4}$-spinel, $\mathrm{LiCoO}_{2}$ or with $\mathrm{LiFePO}_{4}$ [227]. These were mixed using 2 wt.\% gelatin and $\mathrm{LiOH}, \mathrm{NaOH}$ or $\mathrm{KOH}$ to disperse the $\mathrm{CB}$ in the composites. CB had a more uniform distribution onto the oxide and phosphate particles than mechanically mixed components. This gelatin method resulted in cathode materials with 
higher reversible capacities, capacity retention at various rates, and cycling stability than the mechanically mixed composites. This effect of even more pronounced at lower loadings of $\mathrm{CB}$, i.e. $2 \mathrm{wt} . \%$, versus higher loadings of $10 \mathrm{wt} . \%$. Finally, the higher $\mathrm{pH}$ of 12 favored better distribution of $\mathrm{CB}$ particles than at $\mathrm{pH}$ of 9 , and resulting composites demonstrated better cathode particle coating by $\mathrm{CB}$, and consequent enhanced electrical conductivities.

As anode materials, CBs are also of major interest as conductive filler in carbon-carbon or carbon-ceramic composites. As an example of carbon-carbon composites, so-called graphitized sucrose-based carbon microspheres containing CB (GPCMs) have been investigated as an anode material for LIB [228]. After thermal treatments at $2800{ }^{\circ} \mathrm{C}$, anode materials had a reversible capacity of $480 \mathrm{mAh} / \mathrm{g}$ at a current density of $50 \mathrm{~mA} / \mathrm{g}$, which exceeds that reported for graphite microspheres (GM) of $372 \mathrm{mAh} / \mathrm{g}$. During the thermal treatment process, it has been verified that the ordering of the turbostratic graphene sublattice from CB improved, while that of sucrose-derived carbon (a hard-carbon) retained large degree of carbon lattice defects and stacking mismatch. The $\mathrm{d}_{002}$-spacing determined by HRTEM for the CB component was $0.339 \mathrm{~nm}$, still greater than the spacing expected for graphitic materials. The GPCM composites however, provided a more continuous path for electrical conductivity between CB particles. Consequently, the electrodes prepared with graphitized nanocomposites displayed lower series resistance than GM electrodes, or CB thermally treated at $2800{ }^{\circ} \mathrm{C}(\mathrm{GCB})$ alone. Besides the enhanced electrical conductivity properties contributing to the superior reversible capacity, the core-shell structure of the GPCM composites offered extra Li-ion storage sites and allowed for faster Li-ion diffusion compared to GMs and GCBs.

For carbon-oxide composites, $\mathrm{ZnO}-\mathrm{CB}(\mathrm{AB})$ composites have been prepared by ALD and tested as anodes in LIB [229]. Cells reached a capacity of $2096 \mathrm{mAh} / \mathrm{g}$ at $100 \mathrm{~mA} / \mathrm{g}$, and reversible capacity of $1080 \mathrm{mAh} / \mathrm{g}$ at $2000 \mathrm{~mA} / \mathrm{g}$. A stable reversible capacity of $1026 \mathrm{mAh} / \mathrm{g}$ was reached after 500. The reversible capacity of nanocomposites is comparable to the theoretical capacity for $\mathrm{ZnO}$ anode of $987 \mathrm{mAh} / \mathrm{g}$. In these nanocomposites, strong $\mathrm{ZnO}$ interactions with $\mathrm{CB}$ surfaces prevent large volume changes in the $\mathrm{ZnO}$ lattice during Liintercalation and deintercalation. The large volume changes in $\mathrm{ZnO}$ during charge-discharge cycles and its poor electrical conductivity have hindered its applications for batteries. In the nanocomposites, CB enhanced electrical contact between particles, whereas, oxygen vacancies in the $\mathrm{ZnO}$ surfaces allowed for faster $\mathrm{Li}$-ion transport. Other $\mathrm{ZnO}-\mathrm{CB}$ nanocomposites have been investigated for Li-air battery [225]. These studies have reported 
that while pure $\mathrm{ZnO}$ had a capacity of $556 \mathrm{mAh} / \mathrm{g}$ and power density of $17 \mathrm{~mW} / \mathrm{cm}^{2}$, the addition of 2 wt.\% CB Super P resulted in an increase to $776 \mathrm{mAh} / \mathrm{g}$ in capacity and final power density of $20 \mathrm{~mW} / \mathrm{cm}^{2}$. The latter capacity value is much closer to the theoretical one of $820 \mathrm{mAh} / \mathrm{g}$.

Other high-energy density storage device of interest is the Lithium-Silicon battery. The latter battery has a theoretical capacity of $4200 \mathrm{mAh} / \mathrm{g}$ and high potential of $0.4 \mathrm{~V}$ versus $\mathrm{Li} / \mathrm{Li}^{+}$ [225]. This device has its commercialization hampered by the electrode expansion and contraction during Li-intercalation-deintercalation in the $\mathrm{Si}$ crystal. In order to prevent $\mathrm{Si}$ degradation during battery cycling, $\mathrm{Si}$ has been deposited in the large $\mathrm{CB}$ textural (interparticle) pores [230]. Si and CB were prepared by solution plasma processing of $\mathrm{Si}$ suspension in benzene and xylene as carbon precursor. Si-particle size ranged from 50nm to $200 \mathrm{~nm}$, and the CB SSA was $\sim 200 \mathrm{~m}^{2} / \mathrm{g}$. The charge and discharge of a battery are governed by $\mathrm{C}$-rates. The capacity of a battery is commonly rated at $1 \mathrm{C}$, meaning that a fully charged battery rated at $1 \mathrm{Ah}$ should provide $1 \mathrm{~A}$ for one hour, $0.5 \mathrm{~A}$ for two hours, and so on. For these composites, the first cycle capacity was of $\sim 1000 \mathrm{mAh} / \mathrm{g}$, with $55 \%$ coulombic efficiency, meaning a reversible capacity of $553 \mathrm{mAh} / \mathrm{g}$ at a fast cycling rate of $1 \mathrm{C}, 801$ $\mathrm{mAh} / \mathrm{g}$ at $\mathrm{C} / 2$, and finally $1005 \mathrm{mAh} / \mathrm{g}$ at the slowest rate of $\mathrm{C} / 5$. Cells were stable for up to 50 cycles, and final capacities dropped to $260 \mathrm{mAh} / \mathrm{g}$.

Moreover, CB has been intensively investigated as cathode material for the high-power and high-energy density Lithium-Sulfur battery (Li-S) [231]. In these studies, CB (N330) was functionalized with $\mathrm{O}$ and $\mathrm{N}$ species that could strongly interact with $\mathrm{S}$ and prevent its dissolution as polysulfides, a well-known issue for this particular device. N-sites were introduced by $\mathrm{NH}_{3}$ activation. Whereas $\mathrm{O}$-groups were introduced via activation with $\mathrm{CO}_{2}$. Both activation methods additionally introduced micropores by etching the amorphous carbon fractions present the neat CB surfaces, as illustrated in Figure 40. A control sample was prepared by activating $\mathrm{CB}$ in a $\mathrm{CO}_{2} / \mathrm{H}_{2}$ mixture, thus eliminating the O-functionalities while introducing microporosity to the carbon. Final surface areas were $\sim 1300 \mathrm{~m}^{2} / \mathrm{g}$, whereas the total pore volume reached $1.2 \mathrm{~cm}^{3} / \mathrm{g}$; the average pore width for internal micropores and textural pores was in the range of $3 \mathrm{~nm}$ to $4 \mathrm{~nm}$ for all materials. While $\mathrm{N}$-doping significantly improved the electrochemical performance of $\mathrm{CB}$, O-doping deteriorated the battery performance due to loss of electrical conductivity of neat $\mathrm{CB}$, and to unwanted reactions with $\mathrm{S}$. N-doped electrode cells exhibited a reversible capacity of $1020 \mathrm{mAh} / \mathrm{g}$ at $0.1 \mathrm{C}$ rate and $93 \%$ coulombic efficiency. On the other end, O-doped electrode cells had a 
capacity of $550 \mathrm{mAh} / \mathrm{g}$, whereas $\mathrm{CO}_{2} / \mathrm{H}_{2}$ treated materials afforded cells with $700 \mathrm{mAh} / \mathrm{g}$ for the same charge-discharge rate. Non-functionalized acetylene black (AB) mixed with $\mathrm{S}$ was reported to have a discharge capacity of $563 \mathrm{mAh} / \mathrm{g}$ at $0.1 \mathrm{C}$ and $71 \%$ capacity retention at $0.1 \mathrm{C}[232]$.
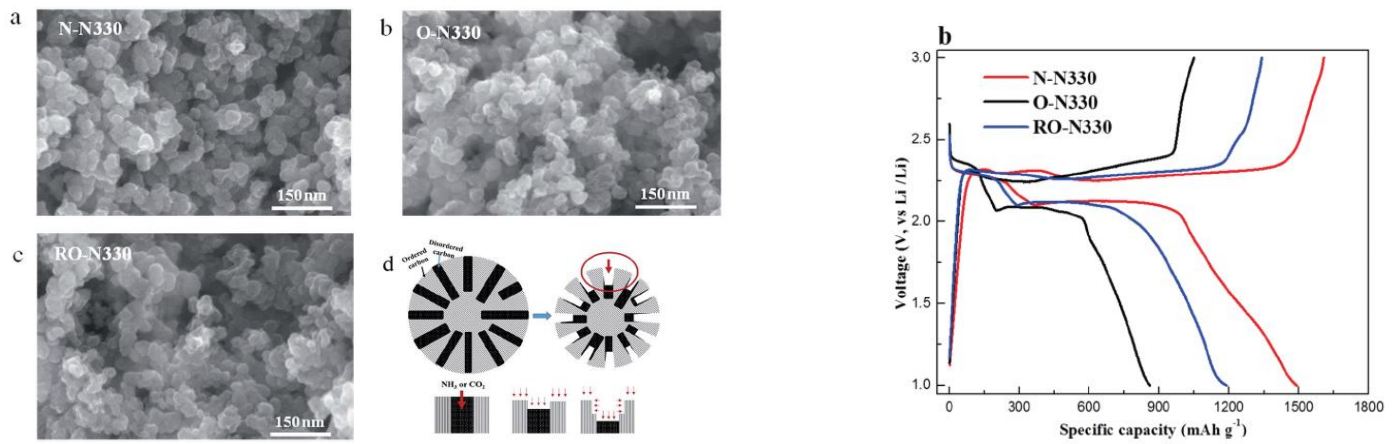

Figure 40 - Left: FESEM images of N-doped N330 (a), O-doped N330 (b), and N/O-doped N330 (c). Right: First Galvanostatic charge-discharge profiles of assembled Li-S cells using $\mathrm{N}$-doped, O-doped, and $\mathrm{CO}_{2} / \mathrm{H}_{2}$ treated $\mathrm{CBs}$ as active electrode materials. Adapted from reference [231].

Edge functionalized $\mathrm{CB}$ particles $(\mathrm{AB})$ prepared by high energy ball-milling under $\mathrm{Ar}$ atmosphere have also been investigated [233]. This process partially exfoliates CB outer layers, as seen in Figure 41, and SSA increased from $60 \mathrm{~m}^{2} / \mathrm{g}$ for CB up to $170 \mathrm{~m}^{2} / \mathrm{g}$ after ball-milling. $\mathrm{CB}$ and functionalized $\mathrm{CB}$ were then impregnated with $\mathrm{S}$ and tested in battery cells. While CB-S electrode cell had a capacity of $453 \mathrm{mAh} / \mathrm{g}$, the exfoliated CB-S composite had a capacity of $814 \mathrm{mAh} / \mathrm{g}$ after 200 cycles and $0.2 \mathrm{C}$ rate. The increased performance was due to the increased SSA of the exfoliated material, which shortened the Li-diffusion pathways, while retaining the good electrical conductivity of CB.

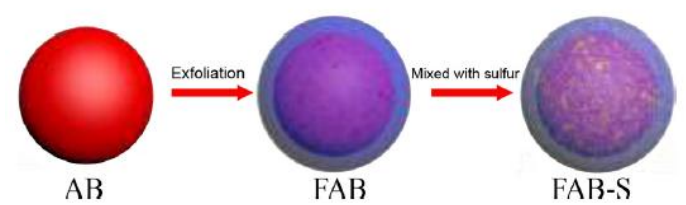



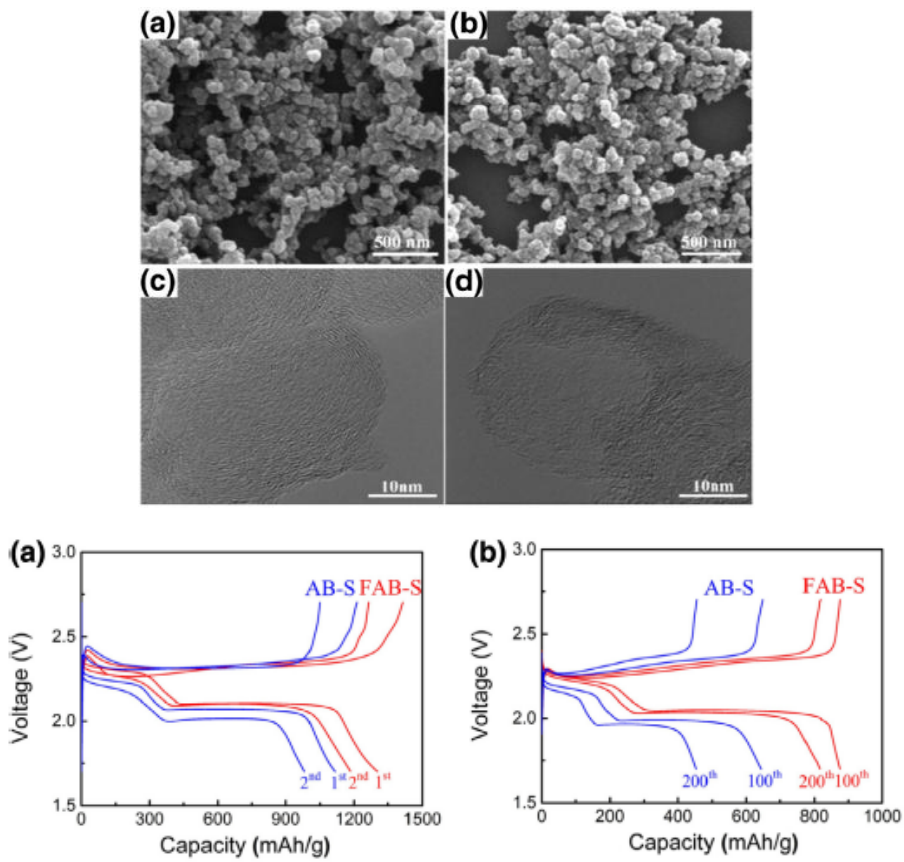

Figure 41 - Top: Scheme illustrating the fabrication of edge-functionalized acetylene blackS nanocomposites. Center: SEM images of acetylene black (a), ball-milled acetylene black (b) and respective TEM images in (c) and (d). Bottom: Galvanostatic discharge-charge profiles of the as-fabricated acetylene black-S (AB-S) and ball-milled edge-functionalized acetylene black-S (FAB-S) composites at a current density of $0.2 \mathrm{C}$ at various cycles. Adapted from reference [233].

Typical battery anodes and cathodes are prepared from powder materials, which require a binder for casting electrode films onto the current collectors. Issues of polymeric binders include their electric insulating properties and pore blockage of the active materials. To reverse the loss of electrical conductivity, $\mathrm{CB}$ is added as conductive filler, whereas pore blocking effects have proven more difficult to probe and solve. In order to investigate the latter, high-surface area $\mathrm{CB}$ particles were mixed the poly(vinylidene fluoride), (PVDF), binder, widely used for electrochemical energy storage devices, in dimethoxyethanedioxolane solvent mixture, or mixed with poly(vinylidene difluoride-cohexafluoropropylene, (PVDF-HFP), in NMP solvent [234]. The CB SSA was $\sim 1100 \mathrm{~m}^{2} / \mathrm{g}$ and particles had a total pore volume of $1.74 \mathrm{~cm}^{3} / \mathrm{g}$. Results indicate that due to poor solubility of PVDF, which hindered access of electrolyte to the cathode particles. When using PVDF-HFP in the same solvent mixture or in NMP, the copolymer had much higher solubility, which improved copolymer swelling and resulted in better electrochemical performance. The latter effect was also observed for poly(ethylene oxide) polymer (PEO). 
The PEO binder, however, led to irreversible capacity loss after each cycle. The highest capacity was obtained for a cell assembled without binder. Nonetheless, the electrode collapsed after the first few cycles [234]. Similarly, Li-S batteries assembled using AB electrode cell have been compared to a lamellar carbon electrode using PVDF binder [235]. While lamellar carbon cells had a stable reversible capacity of $787 \mathrm{mAh} / \mathrm{g}$ at $0.2 \mathrm{C}$ rate and 90\% coulombic efficiency, the CB cell reached a maximum reversible capacity of 334 $\mathrm{mAh} / \mathrm{g}$ after 100 cycles. Results point to the fact that the higher surface accessibility of the lamellar carbon electrode prevented the detrimental pore blocking effects of the binder, as opposed to CB. Finally, the effect of Shewinigan Black addition to LIB graphite anode has been quantitatively investigated, as well as binder, and the electrolytes $\mathrm{LiBF}_{4}$ and $\mathrm{LiPF}_{6}$ [236]. For $\mathrm{LiMnO}_{2}$ as cathode in the cell, reversible capacities for CB electrodes were 180 $\mathrm{mAh} / \mathrm{g}$ in the $0.01 \mathrm{~V}$ to $1.5 \mathrm{~V}$ window, but with $30 \%$ coulombic efficiency. First cycle irreversible capacities further increased from $\sim 25 \%$ to $\sim 70 \%$ as $\mathrm{CB}$ percent weights in graphite were increased from $10 \mathrm{wt} . \%$ up to $90 \mathrm{wt} . \%$. Although SEI stability was comparable between $\mathrm{CB}$ and graphite, but its degradation was stronger over CB. For PVDF binder, additional reduction reactions were observed at $0.35 \mathrm{~V}$. Oxidation reactions were observed between non-fluorinated binders with $\mathrm{CB}$ and $\mathrm{LiPF}_{6}$ in the cathodic region of 3.0-5.5 V. Interestingly, these could be avoided by switching to $\mathrm{LiBF}_{4}$.

When $A B$ was directly ball-milled with elemental sulfur $\left(S_{8}\right)$, composites were found to have uniform $S$ coating the carbon particles. The latter were further coated with sucrose-based carbon, and subsequently deoxidized [237]. This additional carbon coating was added to prevent dissolution of polysulfides in the Li-S battery cells. Indeed, capacities for the coated CB-S particles exceeded $1000 \mathrm{mAh} / \mathrm{g}$ at current densities of $100 \mathrm{~mA} / \mathrm{g}$ or $200 \mathrm{~mA} / \mathrm{g}$ after 6 cycles, whereas that of uncoated CB-S materials dropped to less than $800 \mathrm{mAh} / \mathrm{g}$ at 200 $\mathrm{mA} / \mathrm{g}$.

Finally, potential improvement in CB-based battery anodes and cathodes may come from the use of hollow CB particles [42]. These have been reported after extensive oxidation of CB in $\mathrm{HNO}_{3}$ and subsequent reduction with hydrazine [120] Given the good electrical conductivity of the resulting hollow $\mathrm{CB}$ particles, a potential application includes the Li-S batteries as $\mathrm{S}$ deposition in inner pores may prevent its dissolution as polysulfides and shuttling during battery cycling [238]. Similarly, for batteries where electrode materials undergo fragmentation due to lattice expansion, such as $\mathrm{Si}$ and $\mathrm{ZnO}$ [225, 239-243], small nanoparticles could be grown within the hollow CB cores. This modification could lead to 
mechanically stable nanoparticles that would not suffer from the lattice expansion of Si or $\mathrm{ZnO}$ issues of bulkier crystals due to Li-intercalation during battery cycling. In addition to the good electrical conductivity, these hollow CB particles have more uniform inner pore widths and restricted access by the micropores in the shells. These properties prevent the deposition of polymeric binders within these pores, as it is observed on the external textural pores. Thus, a combination of these properties, indicate new possibilities for high-energy and high-power battery technologies based on CB and CB-based composites.

While Li-batteries have dominated the market for portable electronics and EVs, few countries control nearly all the economically viable Li resources in the world [244]. Thus, there has been a need for identifying more widely available and inexpensive cations to guarantee the future of the market for rechargeable batteries. One of these metals is Sodium (Na), which is widely abundant, inexpensive and it is relatively lightweight. In this direction, carbon anode materials for sodium ion battery (NIB) have been intensively investigated in recent years [225, 245]. These batteries are promising for lightweight, high-power, and inexpensive batteries, and using abundant carbon anodes is of major importance for their popularization. CB materials from Repsol-YPF or Acetylene Black as anode for NIB have been tested versus a $\mathrm{Na}_{0.7} \mathrm{CoO}$ cathode [246]. The reversible capacity of this cell was 200 $\mathrm{mAh} / \mathrm{g}$, and an estimated $0.0155 \mathrm{~mol}$ of Na-ions per $\mathrm{cm}^{3}$ of $\mathrm{CB}$ could be reversibly intercalated-deintercalated. Further improvements in the capacity of these cells, compared to $280 \mathrm{mAh} / \mathrm{g}$ reversible capacity for hard-carbon anode, can be achieved by increasing the surface area and intrinsic porosity of CB-based anodes. The latter can further prevent the deposition of metallic sodium in the textural pores.

\subsection{Supercapacitors}

Electrical double layer capacitor (EDLCs), or simply supercapacitors, are energy storage devices with complementary properties to batteries. Supercapacitors have much higher power densities than batteries, but with lower energy densities than the latter devices [65]. Supercapacitor are composed of an electrically conductive cathode and anode, which are separated by an insulating membrane, and by an electrolyte. In supercapacitors, energy is stored in the electrical double layer (EDL) that form on the surface of the electrodes when an electric potential is applied. When the potential is removed, energy is released and electrons flow through the external circuit connecting the cathode and the anode. Similar to ceramic capacitors, the capacitance depends directly on the surface area of the electrodes. Since the electrolytes wet the surfaces of the electrodes in supercapacitors, high surface area electrodes 
are desired. Most surface area results from micropores, which are pores smaller than $2 \mathrm{~nm}$ in width. Whereas accessibility is often provided by larger mesopores, pores with widths between 2 and $50 \mathrm{~nm}$, and by macropores that are larger than $50 \mathrm{~nm}$ [247]. Thus, hierarchical pore structure is instrumental for balancing surface area and pore accessibility for optimum device performance [248]. Moreover, the power and the energy densities are proportional to the cell voltage and to the square of the voltage, respectively. The electrode and electrolyte stability during charge-discharge are key elements to prevent cell decomposition during cycling. Hence, aqueous electrolytes are limited by the electrolytic decomposition of water, $\sim 1.1 \mathrm{~V}$. Organic electrolytes extend this potential range to $3.5 \mathrm{~V}$, even though bulkier cations and anions have limited access to the micropores and consequently these devices have lower capacitance than those operating with aqueous electrolytes. The energy density of supercapacitors can also be improved with electrochemically reactive groups, as in pseudocapacitors, making these devices potential substitutes for batteries in many applications [249]. The requirements for electrode materials are: good electrical conductivity, hierarchical pore structure, chemical stability and ability to functionalize materials surfaces with redox active groups or even prepare pseudocapacitive nanocomposites with metal oxides of $\mathrm{Mn}, \mathrm{Re}, \mathrm{Ru}$ and Ir [248, 250, 251]. Among all carbon nanomaterials and nanostructured materials, $\mathrm{CB}$ has attracted considerable attention for its electrical conductivity, not to mention its availability and low cost.

Initial reports on CB (Pure Black 205) as the active electrode material in supercapacitors, indicate low gravimetric capacity, $2 \mathrm{~F} / \mathrm{g}$ for organic electrolytes compared to thermally treated nanodiamonds (ND), $35 \mathrm{~F} / \mathrm{g}$, or multiwalled carbon nanotube (MWNT), 11F/g under similar cell preparation conditions [252]. Similar to ND, the electrical conductivity of CB (N326, N121 and P366-E) has been found to increase upon thermal treatments between $1100-1600{ }^{\circ} \mathrm{C}$ [253]. Whereas thermal treatments above $1600{ }^{\circ} \mathrm{C}$, i.e. $2800{ }^{\circ} \mathrm{C}$, resulted in decreased electrical conductivities and in lower specific surface areas for CB. Between 1100 ${ }^{\circ} \mathrm{C}$ and $1600{ }^{\circ} \mathrm{C}$, disordered carbon domains in $\mathrm{CB}$ rearrange into a stacked curved graphene lattice. Further increase in temperatures lead to conversion of curved shells into flat domains [253]. This behavior of $\mathrm{CB}$, as previously discussed [15-17], differs from that of thermally treated ND, which converts into spherical onion-like carbon (OLC) particles [252]. Conversion from $\mathrm{sp}^{3}$ into $\mathrm{sp}^{2}$ carbon starts from the outer ND particle layers, with subsequent complete formation of graphitic onion structure having continuous $\mathrm{sp}^{2}$ carbon shells; polygonization of the outer particle layers then take place with graphitization at high 
temperatures $[252,254,255]$. Furthermore, while specific surface areas of thermally treated $\mathrm{ND}$ are essentially retained upon conversion into OLC, that of $\mathrm{CB}$ has been reported to decreased drastically. Such low specific surface areas result in the low capacitance values reported for $\mathrm{CB}$, as ND thermally treated at $2000{ }^{\circ} \mathrm{C}$ still exhibit a capacitance of $\sim 23 \mathrm{~F} / \mathrm{g}$ [252].

Surface oxidation of $\mathrm{CB}$, such as furnace black, using $\mathrm{O}_{3}$ or $\mathrm{NO}_{2}$ have been found to increase surface acid sites such as phenol groups and lactone groups [200]. The final capacitance of these carbons was largely improved due to added pseudocapacitive contributions of quinone type groups formed within $0.1 \mathrm{~V}$ and $0.5 \mathrm{~V}$ in $\mathrm{H}_{2} \mathrm{SO}_{4}$, especially after $\mathrm{O}_{3}$ treatment. Pseudocapacitive contributions were even more pronounced in activated CB having high SSA.

Most CB grades have much lower surface areas than most activated carbons. While the latter exhibit high capacitance, the former class of materials have considerably higher electrical conductivity, a property that is desirable for fast charge and discharge rates. Similar to GDL and some battery electrodes, CB has been found as an excellent component in high SSA "brick and mortar" type carbon-carbon nanocomposite electrode materials for supercapacitors. Examples include Acetylene Black mixed with GO under ultrasonic conditions, with GO being subsequently converted to rGO [256]. This procedure yielded a composite with $\mathrm{CB}$ anchored in rGO basal planes, acting as pillars of a layered mesoporous composite. When $\mathrm{CB}$ was directly mixed with $\mathrm{rGO}, \mathrm{CB}$ was found in rGO edge sites. Nanocomposite specific surface areas ranged from $500 \mathrm{~m}^{2} / \mathrm{g}$ to $600 \mathrm{~m}^{2} / \mathrm{g}$, whereas that of single component rGO was $\sim 270 \mathrm{~m}^{2} / \mathrm{g}$. Consequently, the reported capacitances for aqueous $\mathrm{KOH}$ electrolyte increased from $69 \mathrm{~F} / \mathrm{g}$ for $\mathrm{rGO}$ to $175 \mathrm{~F} / \mathrm{g}$ for nanocomposites. Composites further exhibited good cycling stability and capacitance retention at various rates, consequence of the low series resistivity of the cells, 0.7 Ohms. The latter may be attributed to the good electrical contact between $\mathrm{CB}$ and $\mathrm{rGO}$ which was instrumental for the high electrical conductivity of nanocomposites.

CB-rGO fibers have also been prepared by wet-spinning method, as shown in Figure 42 [257]. In the latter composites, VULCAN XC-72R was also found to act as pillars for the layered rGO lattice, and SSAs raised with increasing CB content up to roughly $255 \mathrm{~m}^{2} / \mathrm{g}$ at $40 \mathrm{wt} . \% \mathrm{CB}$. Evidence for constricted mesopores was provided by the $\mathrm{N}_{2} 77 \mathrm{~K}$ sorption isotherms (not shown), whereas neat rGO fibers were nonporous and had SSA of $13 \mathrm{~m}^{2} / \mathrm{g}$. These were tested with $\mathrm{H}_{3} \mathrm{PO}_{4}$ electrolyte in PVA, for which capacitance was $98 \mathrm{~F} / \mathrm{g}$, with 
$96 \%$ retention after 2000 cycles. The energy density reported was of $2.8 \mathrm{mWh} / \mathrm{cm}^{3}$, and 1200 $\mathrm{mW} / \mathrm{cm}^{3}$ power density. Compared to rGO fibers, composites displayed much higher capacitance, and capacitance retention, with adjustable charge-discharge properties based on the $\mathrm{CB}$ content. For instance, increasing the amount of $\mathrm{CB}$ resulted in lower electrolyte diffusion resistance, and electrochemical series resistance (ESR). Higher CB contents also translated into flexible composites with enhanced mechanical strength compared to rGO fibers. Thus, CB composite fibers have potential for uses in wearable electronic devices. 

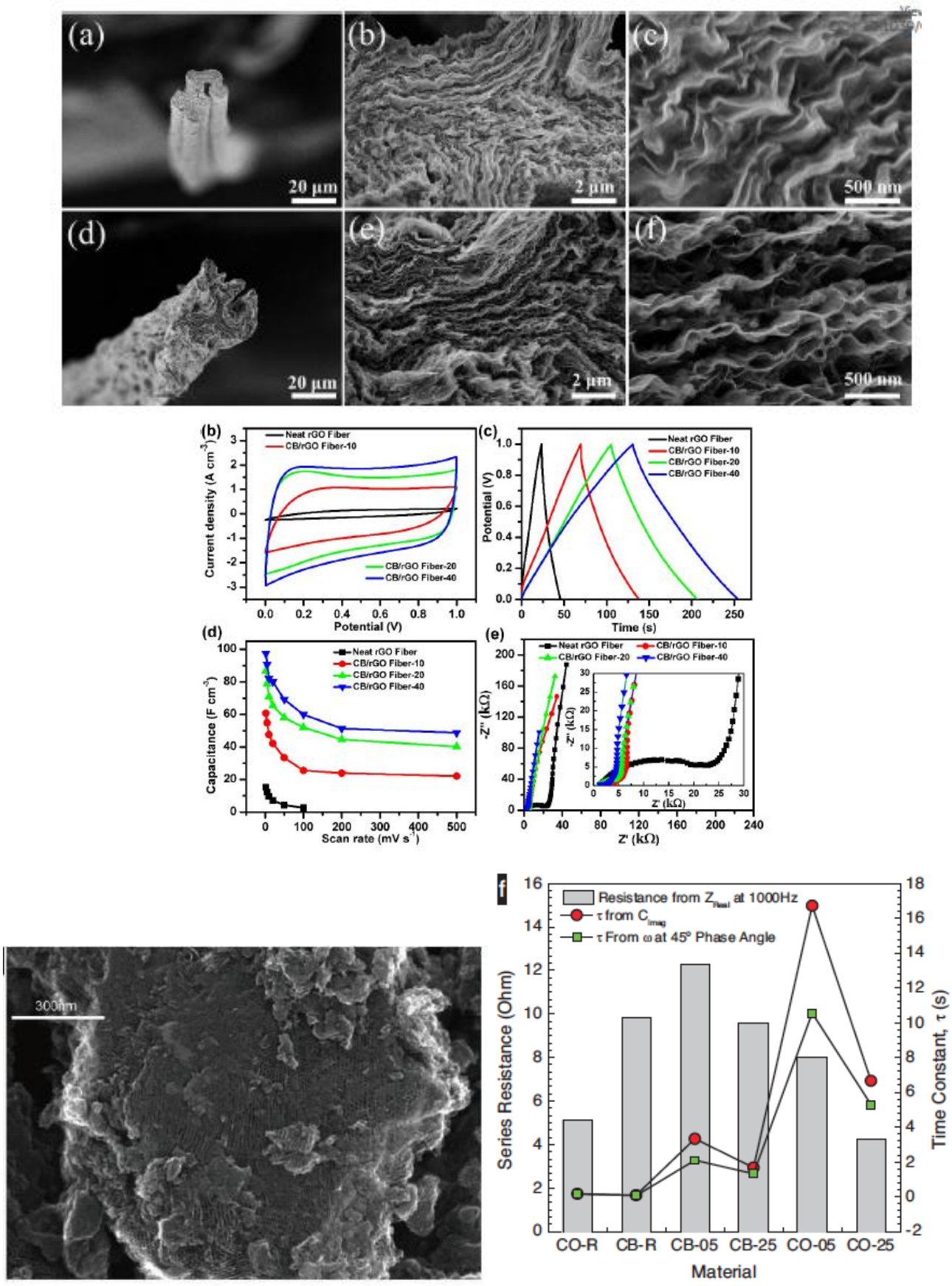

Figure 42 - Top: SEM images of cross-sections of rGO (a-c) and of CB-rGO with 40 wt.\% $\mathrm{CB}$ nanocomposite fibers. Bottom: Cyclic voltammograms (CVs) at $50 \mathrm{mV} / \mathrm{s}$ (b), galvanostatic charge-discharge $(\mathrm{GCD})$ at $0.64 \mathrm{~A} / \mathrm{cm}^{3}$ (c), volumetric capacitance at various scan rates (d) and Nyquist plots of the various nanocomposites in comparison to rGO fibers; inset shows the high frequency region of the Nyquist plots (e). Adapted from reference [257]. Bottom Left: SEM image of CB-OMC composite prepared by the "brick and mortar" softtemplating method with 25 wt.\% CB in the synthesis gel. Bottom Right: Calculated ESR and 
time constants for various $\mathrm{CB}-\mathrm{OMC}$ and $\mathrm{OLC}-\mathrm{OMC}$ nanocomposites in comparison to $\mathrm{CB}$ or OLC (CO) supercapacitor electrode materials. Adapted from reference [258].

Other high surface area nanocomposites have been reported for $\mathrm{CB}$ with a soft-templated ordered mesoporous carbon (OMC) framework [258]. The CB-OMC materials were prepared following the "brick and mortar" method [259], with CB serving as highly electronically conductive carbon "bricks" interconnected by the high surface area OMC "mortar". The OMC forms via self-assembly, in situ polymerization and microphase separation of a resorcinol-formaldehyde resin with a triblock copolymer of general formula poly-ethylene oxide-poly-propylene oxide-poly-ethylene oxide (PEO-PPO-PEO) that acts as structure directing agent. In the synthesis gels, CB particles were found to interact with the hydrophilic PEO blocks and the resorcinol. This interaction allowed for its dispersion within the resorcinol-formaldehyde resin in the phase-separated polymer composite. After thermal treatments under inert atmosphere, the resin thermosets and converts into a carbon material, whereas the PEO-PPO-PEO polymer decomposes, leaving an open structure of mesopores resembling that of the polymer mesophase. Due to the presence of $\mathrm{CB}$, the effective size of the triblock copolymer micelles increases. Consequently, by increasing the ratios of $\mathrm{CB}$ in the synthesis gels, the mesopore widths of final CB-OMC composites also increased from $\sim 6$ $\mathrm{nm}$ in the neat OMC up to $\sim 17 \mathrm{~nm}$ in composites having $50 \mathrm{wt} . \% \mathrm{CB}$. At CB ratios less than 25 wt.\% the conductive "bricks" were dispersed throughout the OMC materials as seen in Figure 42. At $50 \mathrm{wt} . \%$ and higher ratios of "bricks", thin mesoporous films coating and interconnecting the CB particle aggregates formed. The SSA of composites were varied from $200 \mathrm{~m}^{2} / \mathrm{g}$ to $700 \mathrm{~m}^{2} / \mathrm{g}$, which is far higher than most graphitic carbons. Capacitance values were comparable to that of mesoporous carbon materials with similar SSA. Another effect of increasing $\mathrm{CB}$ ratio was the decrease in series resistance of the supercapacitor cells assembled. Consequently, CB-OMC composites had better capacitance retention at fast charge-discharge rates than neat OMC or with CB loadings of $5 \mathrm{wt} \%$ or less. Similar effect was found for OLC "bricks" used in the same study [258].

Besides carbon-carbon composites, conductive composites of $\mathrm{CB}$ with polymers with fibrous and segregated particle morphologies have been investigated. Core-shell composite nanospheres of CB and polypyrrole (PPY) have been prepared in the presence of poly(2hydroxy-3-(methacryloyloxy) propane-1-sulfonate), (PHMAS), that act as both surfactant and as sulfur dopant [260]. Nanocomposites displayed good electrical conductivity, with 
maximum values found for a 20 wt.\% loading $\mathrm{CB}$, which also had the lowest $\mathrm{S} / \mathrm{N}$ ratio. Materials have semiconducting properties, with the electrical conductivity of samples increasing with increasing temperature. Capacitance values for neat PPY nanoparticles in aqueous $\mathrm{NaNO}_{3}$ electrolyte was $\sim 120 \mathrm{~F} / \mathrm{g}$, which increased to $366 \mathrm{~F} / \mathrm{g}$ for composite with 20 wt.\% CB. The capacitance dropped to $210 \mathrm{~F} / \mathrm{g}$ for higher CB loadings. Composites exhibited good cycling stability and capacitance retention at current densities of up to $5 \mathrm{~mA} / \mathrm{cm}^{2}$, and various charge-discharge rates. Finally, electrochemical series resistivities were between 3 and $8 \mathrm{Ohms}$, similar to those reported for CB-OMC composites. Other composite examples include the mechanically mixed CB (CDX975) with poly(methyl methacrylate), (PMMA), which yielded a segregated structure with PMMA forming faceted grains and CB particles aligning in a 3D network of nanowires [261]. Composites where transparent up to $1 \mathrm{wt} . \%$ of $\mathrm{CB}$ in PMMA. In contrast, solution mixing yielded randomly distributed $\mathrm{CB}$ particles in the bulk PMMA matrix, consequently having higher electronic percolation threshold. Electrochemical impedance measurements indicate that mechanically mixed composites have similar electrical conductivity to SWNT as conductive fillers. Although only electrical conductivity properties were measured for CB-PMMA composites, these are promising for flexible capacitive energy storage in electronic displays, sensors and other portable electronic devices.

Beyond conventional supercapacitors, electrochemical flow capacitor (EFC) devices have been the subject of recent investigation for large-scale energy storage [262]. Different than supercapacitors that utilize liquid electrolytes and electrode films on a current collector, EFC make use of flowable porous carbon-electrolyte solution slurries. The slurry flows through 2 separated loops, each constituting a half-cell, and with each loop being forced to flow through electrostatically charged plates that act as current collectors. At this stage, each halfcell is separated by a membrane that allows dissolved counter ions to diffuse through, thus balancing the EDL on the particles on both sides. The charged particles are then stored in separate reservoirs and released as energy is needed. The charge transfer is given by contact between particles, and concentrated slurries with good stability in the electrolyte-solvent system is desired. This stability is guaranteed by the electrical double layer once the electrolyte is adsorbed and immobilized on the particle surfaces. Although the chargedischarge process takes places within several minute intervals, the EFC devices are attractive alternative to large-scale energy storage such as power-grids, where higher energy densities than conventional supercapacitors or batteries can currently provide are desirable. Similar to 
conventional supercapacitors, active carbon particles need to have high surface area for storing charge and good electrical conductivities for charge transfer and polarization in EFC. First reports used activated carbon micron sized spheres as active material [262-265], whereas, graphene has been proposed as an efficient material for EFC [266]. In this direction, graphitized sucrose-based carbon microspheres containing $\mathrm{CB}$ are of interest due to their high SSAs and electrical conductivities [228]. Similarly, mesoporous CB-pillared graphene composites [256] have the potential for active slurry in EFC, as these retain the particle porosity even if stacking of layered particles in a slurry may take place. Additionally, there is vast number of possibilities to improve the electrical conductivity of $\mathrm{CB}$ composites with tertiary particle structures, to modify the surfaces of CB with hydrophilic or hydrophobic polymers, i.e. hydrophilic poly(sodium-4-styrene sulfonate) [267, 268], and pH responsive polymers such as poly(acrylamide-co-vinylamine) [269], for suspending these particles in water, or organic solvents, and dope the surfaces with redox active coatings [133, 207, 260] to increase the energy storage by pseudocapacitive contribution in EFC [270]. Besides ensuring good electrical conductivity, the ability to tailor interparticle and particle-electrolyte interactions, and potentially slurry viscosity for modulated charge-discharge cycles, further increase interest for CB in emerging energy storage technologies [262, 264, 270].

\subsection{Photocatalysts and Solar Devices}

$\mathrm{CB}$ is a good active component in composite electrodes for dye sensitized solar cell (DSSC) and for photocatalysts [271-273]. Photocatalysts, commonly titanium dioxide $\left(\mathrm{TiO}_{2}\right)$ and other semiconducting materials, have bandgaps of energies in the UV-vis region of the electromagnetic spectrum. Light excites electrons from the conduction band to the valence band of the solid producing an electron-hole pair. The hole $\left(h^{+}\right)$and electron $\left(e^{-}\right)$pairs generate free-radicals which promote redox reactions on the photocatalysts surfaces. Photocatalysts properties can be tailored by chemical composition, as well as by their nanoarchitecture [274, 275].

DSSC, or Grätzel cells, also operate with semiconducting materials formed between a photosensitized anode and an electrolyte [276]. The Grätzel cell design has a top transparent anode, either fluoride-doped tin oxide (FTO) or indium tin oxide (ITO) conductive glass. On the back of this conductive plate is a thin layer of sintered $\mathrm{TiO}_{2}$ nanoparticles, which has a mesoporous structure with high surface area. Since $\mathrm{TiO}_{2}$ absorbs only photons in the UV, the plate is then immersed in a mixture of a photosensitive solution of ruthenium-polypyridine dye (molecular sensitizers), i.e. [Ru(4,4'-dicarboxy-2,2'-bipyridine $\left.)_{2}(\mathrm{NCS})_{2}\right]\left(\mathrm{N}_{3}\right)$, that is 
bound to a photoanode via carboxylate moieties, an ester, a chelating, or bidentate bridging linkage. A second conductive plate made of $\mathrm{Pt}$ and coated with a thin layer of iodide electrolyte is then joined and sealed with the FTO or ITO supported electrode to prevent the electrolyte from leaking. In this cell, the dye is excited by light and rapidly injects an $e^{-}$into the $\mathrm{TiO}_{2}$ after light absorption. The $e^{-}$diffuses through the $\mathrm{TiO}_{2}$ particle network to be collected at the front side of the FTO electrode. The dye is regenerated via reduction by a redox shuttle, $\mathrm{I}_{3} / \mathrm{I}^{-}$, dissolved in a solution. Diffusion of the oxidized form of the shuttle to the counter electrode completes the circuit [277].

In photocatalysts, the good electrical conductivity of $\mathrm{CB}$ allows for fast electron transfer and leads to decreased electron-hole recombination in the semi-conducting oxide, i.e. $\mathrm{TiO}_{2}$ [274]. The CB additive further increases the thermal stability of the anatase phase, preventing its crystallization into rutile. Results confirm that indeed, Acetylene Black- $\mathrm{TiO}_{2}$ composite films have been found to exhibit 50\% more activity than $\mathrm{TiO}_{2}$ films alone [278-280]. In all these works, the intimate contact between $\mathrm{CB}$ and $\mathrm{TiO}_{2}$ photocatalyst is instrumental. Thus, nanocomposites prepared by a sol-gel method yielded materials with bang-gaps of $1.3 \mathrm{eV}$, and well-defined radiation absorption in the visible region of the electromagnetic spectrum [279]. The latter had not been obtained for physical mixtures of $\mathrm{CB}$ and $\mathrm{TiO}_{2}$. This results from the abundance of inactive sites found in the physically mixed nanocomposites, as opposed to a more uniform surface found in the sol-gel materials, for which the abundance of hydroxyl surface groups in the sol-gel composites was largely responsible for the long-term stability and activity of the photocatalysts [280]. $\mathrm{CB}-\mathrm{TiO}_{2}$ composites prepared from the solgel method further presented higher photocatalytic activity than activated carbon- $\mathrm{TiO}_{2}$ composites. That was mostly due to the large textural pores found in the $\mathrm{CB}$ composites and to their higher electrical conductivities compared to activated carbon, which favored fast diffusion of dyes to the catalyst surfaces. In addition to electrical conductivity, external polarization studies under $\mathrm{UV}$ irradiation demonstrated uniform response of $\mathrm{CB}-\mathrm{TiO}_{2}$ prepared by the sol-gel method under anodic polarization [281]. This was in contrast to results obtained for physical mixtures of the two components, which had non-uniform responses and with enhancement under cathodic polarization. Capacitive tests under dark conditions revealed larger positive shift in the rest potential of the sol-gel composites plus evidence of Fermi-level pinning due to carbon electronic states with strong acceptor character in the sol-gel derived nanocomposites [281]. 
Parallel to these developments, CB-composites have been investigated as alternative for Pt on FTO or ITO glass counter-electrodes for reduction of $\mathrm{I}_{3}$ in DSSCs [282]. Advantages of CB-FTO over Pt-FTO glass includes the fact that CB is vastly abundant, it is inexpensive, and is corrosion resistant. Other CB-nanocomposites and carbons, namely CB-graphite, CBpolyaniline, $\mathrm{CB}-\mathrm{TiO}_{2}, \mathrm{CB}-\mathrm{Pt}$, activated carbon, and single-walled carbon nanotubes (SWNT) have been reported with results pointing to $\mathrm{CB}$ as the best catalyst material [282]. In fact, PRINTEX $\mathrm{L}$ is an excellent active catalyst for the $\mathrm{I}_{3}$ reduction, with cells reaching an efficiency of $9.1 \%$ under 1 sun in a solvent mixture of acetonitrile and valeronitrile [283]. The short-circuit current $\left(\mathrm{J}_{\mathrm{SC}}\right)$ and open circuit potential $\left(\mathrm{V}_{\mathrm{OC}}\right)$ were $16.8 \mathrm{~mA} . \mathrm{cm}^{2}$ and 789.8 $\mathrm{mV}$, respectively [283]. Results represented an improvement over graphite-CB, for which a $6.67 \%$ photocatalytic efficiency (PCE) with lower $\mathrm{V}_{\mathrm{OC}}$ of $826 \mathrm{mV}$ and $\mathrm{J}_{\mathrm{SC}}$ of $11.34 \mathrm{~mA} . \mathrm{cm}^{2}$ was obtained in acetonitrile solvent [283]. The activity of CB results from the large numbers of edge sites in its short graphene domains, thus explaining the increased activity [283]. CB$\mathrm{TiO}_{2}$ composites have also been found as good catalysts for $\mathrm{I}_{3}$ reduction, with cells reaching 7.4\% efficiency for 5:1 wt. ratio of $\mathrm{TiO}_{2}: \mathrm{CB}$ compared to the $7.7 \%$ PCE of Pt-FTO in similar conditions [282]. Yet, $\mathrm{CB}-\mathrm{TiO}_{2}$ displayed lower charge transfer resistance than Pt-FTO, and comparable to the $8 \%$ PCE found for more conductive Pt-PRINTEX L [284]. Similarly, commercially available activated carbon, despite its high surface area and poor crystallinity, exhibited much lower efficiency than $\mathrm{CB}$, reaching $3.89 \% \mathrm{CE}$ in acetonitrile as the latter lacked graphene edge sites. Hence, there is possibility to further improve the reactivity of activated carbons by precursor selection. As previously mentioned, similar to graphite, CB particles such as acetylene black, furnace black, and others can also be oxidized and by $\mathrm{KMnO}_{4}-\mathrm{H}_{2} \mathrm{SO}_{4}$ solution [18], or by $\mathrm{HNO}_{3}$ and $\mathrm{HNO}_{3}-\mathrm{H}_{2} \mathrm{SO}_{4}$ mixture $[45,120,285]$ and subsequently exfoliated into graphene quantum dots (GQD) with additional OCB nanoparticles $[18,45,120]$. GQD are advanced nanomaterials with structure composed of single layer and multilayer graphene stacks having less than $30 \mathrm{~nm}$ in size, and lateral size larger than particle stacking height. GQD exhibit prolonged quantum confinement effects, stable photoluminescence and excellent chemical stability. Their electronic properties originate from the quantum confinement or conjugated $\pi$-system, the different hybridization of the carbon atoms, presence of surface groups and of covalently bound luminescent molecules, and to crosslink-enhanced emission (CEE) effect [286, 287]. In this direction, high surface area carbon aerogels (CA) coated with GQD from CB precursor have been recently reported, see Figure 43 [285]. The average GQD size obtained from microwave 
assisted oxidation of $\mathrm{CB}$ in $\mathrm{HNO}_{3}-\mathrm{H}_{2} \mathrm{SO}_{4}$ mixture was $10 \mathrm{~nm}$ and with $2 \mathrm{~nm}$ thickness. These were adsorbed on the CA, and subsequently film cast onto FTO glass. The resulting composites not only had activities comparable to Pt-FTO CEs, but greater stability than PtFTO or CA-FTO as assessed by the drop of the cathodic current for the latter 2 materials after 500 cycles. Currents slightly increased for GDQ-CA composites, thus representing a more corrosion-resistant and inexpensive alternative to Pt. Moreover, $\mathrm{N}$-doped graphene has also been proposed as an efficient $\mathrm{I}_{3}$ reduction photocatalyst [288]. Recent study on $\mathrm{N}$-doped multilayer GQD has found that the emission properties can be modulated from $300 \mathrm{~nm}$ to over $1000 \mathrm{~nm}$ [289]. Moreover, the N and O-sites in GQD have been found to catalytically active centers in reduction reactions [290]. Hence, there is a need to develop methods to prepare N-doped GQDs from $\mathrm{CB}$, as these could substantially lower the costs of this novel photocatalyst material.
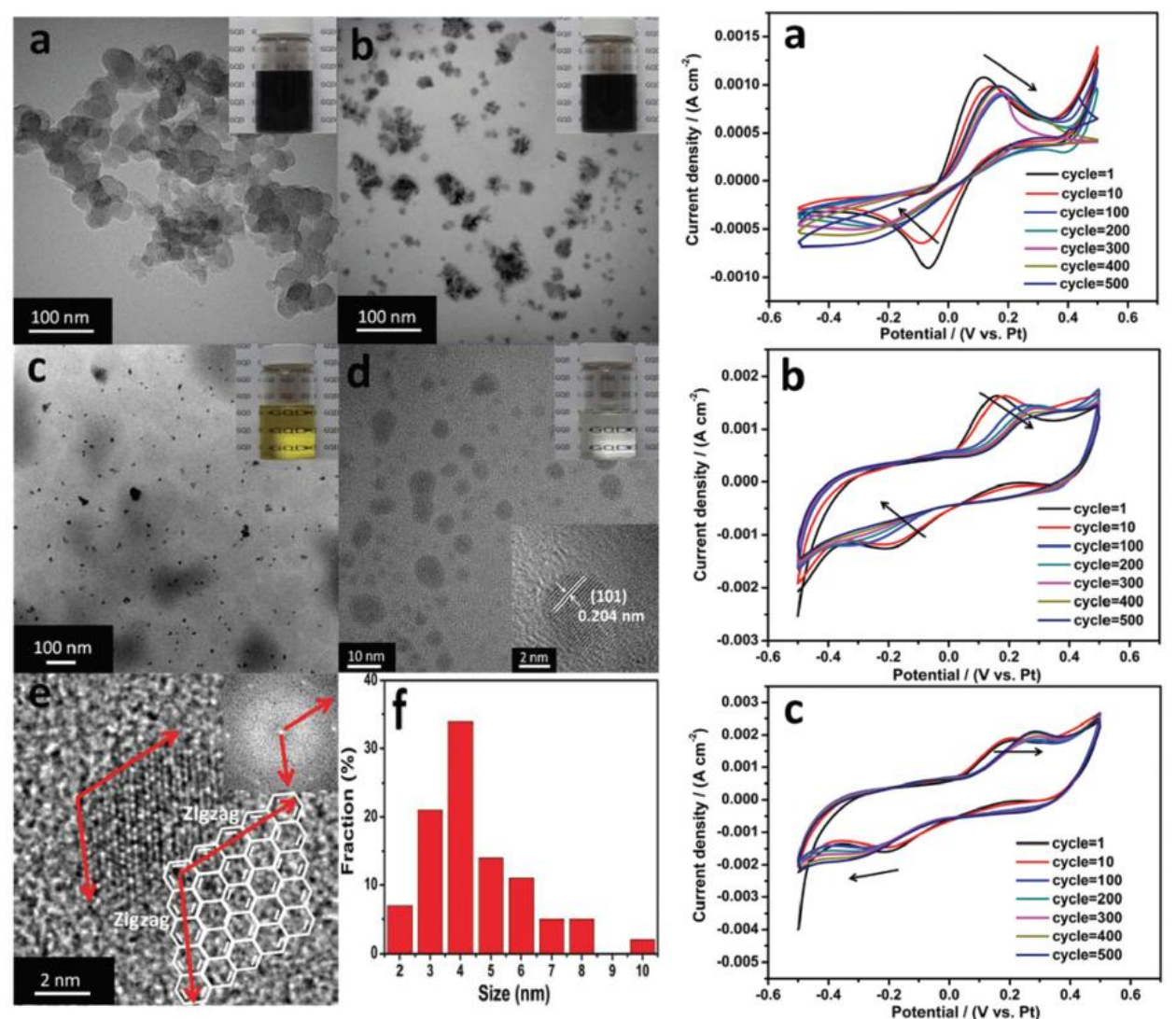

Figure 43 - Left: TEM images of CB (a), exfoliated CB for $5 \mathrm{~min}(\mathrm{~b}), 20 \mathrm{~min}(\mathrm{c})$, and $60 \mathrm{~min}$ (d), with inset pictures of product suspensions. HRTEM of GQD prepared using 60min for exfoliation, and size distribution of GQDs (f). Right: Cyclic voltammograms of Pt (a), carbon 
aerogel (CA) (b), and GQD/CA electrodes recorded for 500 cycles in $1 \mathrm{mM} \mathrm{I}_{2}, 10 \mathrm{mM} \mathrm{LiI}$, and $0.1 \mathrm{mM} \mathrm{LiClO}_{4}$ in $\mathrm{CAN}$ at $25 \mathrm{mV} / \mathrm{s}$ scan rate. Adapted from reference [285].

Another major development for more cost effective DSSC came from the substitution of FTO by stainless steel (SS) as counter electrode substrate, which was possible to attain due to the good interaction of CB with SS, and to the chemical stability of CB [291]. In this work, varying ratios of $\mathrm{CB}$ to epoxy polymer composites were directly cast onto SS current collectors. Optimum ratio of 86 wt. $\% \mathrm{CB}$, a $6.1 \%$ PCE for the $\mathrm{I}_{3}$ reduction was obtained, versus $7.1 \%$ for Pt-FTO, or for Pt-resin composite with 6 wt.\% CB (7.3\%). Besides, at 86 wt.\% $\mathrm{CB}$, electrodes exhibited better stability due to the corrosion protective properties of CB-epoxy resin and provides suitable alternative to replacing Pt-FTO in DSSC.

Besides films, C-based nanofluids have been found as inexpensive alternatives for solar energy conversion, as these nanofluids have enhanced solar absorption properties that can lead to water vapor generation without fuel resources [292, 293]. When N115 was mixed with water with various mass ratios, nanofluids with shear thinning behavior and light absorption between $200 \mathrm{~nm}$ and $2500 \mathrm{~nm}$ were obtained [292]. As shown in Figure 44, CB nanofluids and water have near perfect infrared absorption, whereas CB nanofluids have higher UV-vis light absorption than water. Furthermore, thermal conductivity of fluids could be increased by raising the N115 concentration in the nanofluid composition, given partial particle networking enhances thermal conductivity by reducing fluid interfacial resistance [294]. The combination of the latter properties results in higher fluid temperature and greater temperature enhancement of nanofluid over water. Such properties are also of great interest for the development of solar heaters, and distillation systems which are capable of generating liquid vapor without the requirement of heating the fluid volume [293]. For instance, for the vapor-liquid phase diagram of ethanol and water produced by solar distillation using N115 with $\mathrm{Au}-\mathrm{SiO}_{2}$ core-shell nanofluids (Figure 44), the mole\% ethanol obtained in the distillate, relative to a standard equilibrium distillation curve at $1 \mathrm{~atm}$ and $25{ }^{\circ} \mathrm{C}$, is consistently higher than that obtained by conventional flash distillation. Authors have pointed out that this is due to the hot surfaces of the illuminated nanoparticles induce preferential vaporization of the more volatile component of the mixture. Finally, analysis of the distillate showed no presence of particles nor any evidence of oxidation products or from chemical degradation of ethanol, such as methanol and acetic acid. 

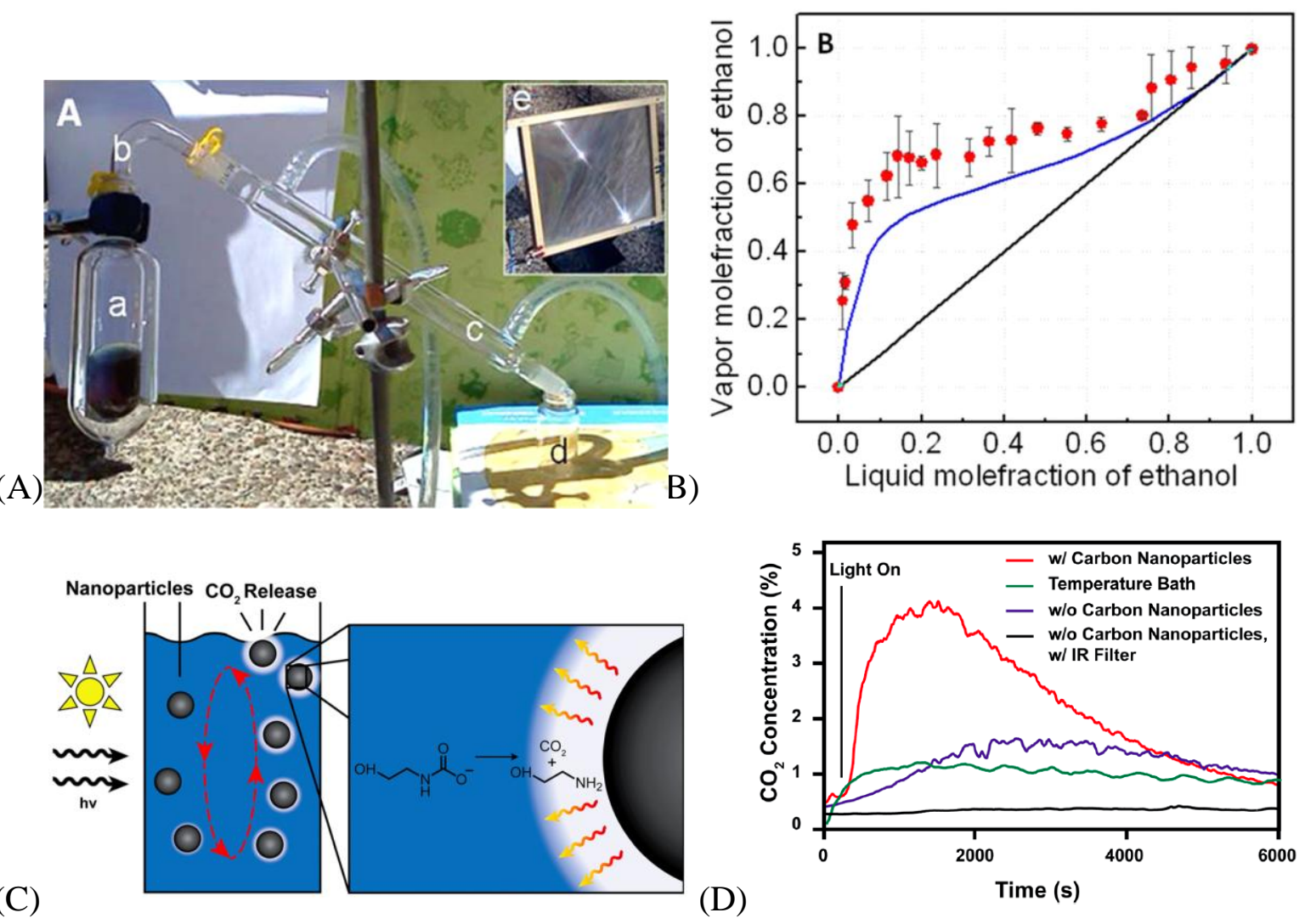

Figure 44 - Photography of an assembled system for the solar distillation of ethanol. The solar distillation apparatus includes (a) vacuum-jacketed glass container, (b) connector tube, (c) water condenser, and (d) fraction collector vessel. The solution was irradiated by a 26.67 x $26.67 \mathrm{~cm}$ Fresnel lens (e) with a $44.5 \mathrm{~cm}$ focal length (inset) in (A). Vapor-liquid diagram of ethanol-water fractions produced by solar distillation. Mole \% of ethanol in vapor phase for $\mathrm{Au}-\mathrm{SiO}_{2}$ nanoparticle alcohol-water mixtures under solar exposure (red dots) and standard equilibrium distillation curve at 1 atm and $25{ }^{\circ} \mathrm{C}$ (blue curve) in (B). The $\mathrm{Au}-\mathrm{SiO}_{2}$ nanoparticle concentration is $2.5 \times 10^{10}$ particles $/ \mathrm{cm}^{3}$. Adapted from reference [293]. CB nanoparticle $\mathrm{CO}_{2}$ regeneration mechanism (C): $\mathrm{CB}$ nanoparticles absorb actinic light and convert it to thermal energy, resulting in a high local temperature near the nanoparticle surface. At the surface, $\mathrm{CO}_{2}$ is regenerated from MEA, forming a gas bubble around the nanoparticle. The bubble can grow large enough to force the nanoparticle to the surface of the fluid and release $\mathrm{CO}_{2}$. Representative data. Release of $\mathrm{CO}_{2}$ is triggered by light in MEA. After $2000 \mathrm{~s}$, maximum $\mathrm{CO}_{2}$ release takes place and the release rate of $\mathrm{CO}_{2}$ slows. Some $\mathrm{CO}_{2}$ is released without carbon black due to the IR transmittance of the light source, resulting in increased bulk fluid temperatures (D). Adapted from references [295, 296]. 
N115-monoethanolamine nanofluids (CB-MEA) in water or 1-butanol have been tested as an energy-efficient alternative for photochemical $\mathrm{CO}_{2}$ release, regenerating monoethanolamine (MEA) in post-combustion $\mathrm{CO}_{2}$ capture technology and tuning $\mathrm{CO}_{2}$ reactivity with light $[295,296]$. As illustrated in Figure 44, the CB particles absorb heat, and a large temperature gradient between the $\mathrm{CB}$ surface and $\mathrm{MEA}-\mathrm{CO}_{2}$ solution arises. The thermal energy is then transferred from the CB particles the solution, thus breaking the bond between MEA and $\mathrm{CO}_{2}$ and regenerating the MEA without the need for heating the system. Consequently, these nanofluids are of great importance for preventing the thermal degradation and MEA evaporation during recycling in post-combustion $\mathrm{CO}_{2}$ capture.

\section{5 $\mathrm{CO}_{2}$ storage and separations}

CCS technologies aim at reducing the $\mathrm{CO}_{2}$ emissions at large emitting sources, as thermoelectrical power plants [297]. These technologies cover the separation of carbon from an effluent gas stream, and its transport to an underground geological formation. Regarding transportation, pipes seem the most cost-effective option for directing $\mathrm{CO}_{2}$ towards plants for mineralization, or to geological storage points, saline aquifers, the ocean, and also driving $\mathrm{CO}_{2}$ towards oil production sites, where it is injected in rocks for enhanced oil recovery [297].

MEA has been proposed for $\mathrm{CO}_{2}$ separation, but it is very corrosive, volatile and difficult to regenerate [297]. Recent research has focused in non-volatile systems that could replace MEA, including room temperature ionic liquids (RTIL) and task specific ionic liquids (TSIL). RTIL and TSIL are promising compounds to substitute MEA in post-combustion systems due to modular properties, less corrosive nature, better thermal stability and negligible vapor pressure than organic amines [298-300]. These compounds, however, have high viscosity and high densities, making handling difficult and limiting the diffusion and solubility of $\mathrm{CO}_{2}$. Porous membranes having functional groups capable of binding to $\mathrm{CO}_{2}$ have emerged as an attractive alternative over liquids [301]. But controlling the surface density and nature of these groups is still challenging, thus reducing the selectivity for $\mathrm{CO}_{2}$ over other post-combustion gases. Consequently, membranes supporting RTIL and TSIL compounds appear as more functional option to maximize the surface areas of high viscosity TSILs and for promoting the interaction of the porous support with $\mathrm{CO}_{2}$.

Similar to mesoporous carbon supports, RTIL have further been found to increase surface polarity of $\mathrm{CB}$ micropores, which is important for developing sorbents for $\mathrm{CO}_{2}$ [302]. Hence, 
RTIL composite membranes with mesoporous carbonaceous materials have high selectivity for $\mathrm{CO}_{2}$ separations and capture in post-combustion industrial emissions containing other less polar gases such as $\mathrm{N}_{2}$ [303-305]. Free-standing mesoporous carbon-CB composite membranes prepared by the "brick and mortar" method, were successfully tested as supports to RTIL for $\mathrm{CO}_{2}$ separations. Similar to powders prepared by the same method and tested for supercapacitors, increasing ratios of $\mathrm{CB}$ to the synthesis gels lead to carbons with enlarged mesopores. The increase in mesopores widths, facilitated the diffusion of gases and maximized the distribution of adsorbed surface RTILs and consequently the accessible active surface area for $\mathrm{CO}_{2}$ binding and the selective of the membranes over $\mathrm{N}_{2}$ [304].

Besides controlled ratios of ultramicropores (pores smaller than $0.7 \mathrm{~nm}$ ) and supermicropores (pores with widths between 0.7 and $2 \mathrm{~nm}$ ) [306], the direct $\mathrm{N}$-doping of porous carbons is another effective way to introduce basic surface groups for $\mathrm{CO}_{2}$ separations [307-311]. Recent findings for $\mathrm{N} 110 \mathrm{CB}$ indicate that $\mathrm{N}$-doping plays major role in $\mathrm{CO}_{2}$ adsorption capacity [302]. Surface areas are relatively low for N110, $143 \mathrm{~m}^{2} / \mathrm{g}$, and $\mathrm{HNO}_{3}$ oxidation increased it to $263 \mathrm{~m}^{2} / \mathrm{g}$. N-doping of oxidized N110 using DETA reduced SSAs of OCB down to $30 \mathrm{~m}^{2} / \mathrm{g}$. The $\mathrm{CO}_{2}$ uptake of the $\mathrm{N}$-doped $\mathrm{CB}$ was investigated by breakthrough method in flowing $\mathrm{CO}_{2} / \mathrm{N}_{2}$ feeds and compared to the pristine and to oxidized N110, all three supporting the (Trihexyl(tetradecyl)phosphonium 1,2,4-triazole), [ $\left.\mathrm{P}_{66614}\right][$ Triz], TSIL. Among the 3 nanocomposites, the uptake of the CB- and OCB-[P $\left.\mathrm{P}_{66614}\right][$ Triz] were approximately the same, $\sim 6.5-6.6 \mathrm{mgCO}_{2} / \mathrm{g}$ sorbent. Despite the considerably lower SSA, the $\mathrm{CO}_{2}$ capacity of the $\mathrm{N}$-doped $\mathrm{CB}$ was double that of the $\mathrm{CB}$ and $\mathrm{OCB}$ based nanocomposites, $\sim 12.8 \mathrm{mgCO}_{2} / \mathrm{g}$ [302]. Although authors claim that micropores in these nanocomposites restricted $\mathrm{CO}_{2}$ accessibility to the binding sites, it is clear by the calculated SSA that micropores and small mesopores in oxidized N110 were blocked during treatment with diethylenetriamine (DETA). More studies on N-doped activated CB having even larger SSA $[212,213]$ are thus necessary for verifying the effect of PSD on the surface availability Nsites and of its interactions with immobilized TSIL. Such studies are of major significance to the design of platforms for cost-effective, large-scale $\mathrm{CO}_{2}$ mitigation technologies.

The importance of grafting $\mathrm{CO}_{2}$ binding groups to accessible regions other than deep micropores of CB composites was recently demonstrated for Cabot BP-2000 and EC 600JD from Akzo Nobel, both before and after steam activation; these were further compared to commercial activated carbons with SSA between 1100 and $2300 \mathrm{~m}^{2} / \mathrm{g}$ [312]. All carbon materials were then modified using polyethyleneimine (PEI) by non-covalent method. The 
resulting molecular basket sorbents (MBS) were tested for the $\mathrm{CO}_{2}$ adsorption under flowing $\mathrm{CO}_{2}$. The SSA of BP-2000 and of EC 600JD were 1486 and $1121 \mathrm{~m}^{2} / \mathrm{g}$, respectively. The SSA of CB-PEI nanocomposites ranged between $320 \mathrm{~m}^{2} / \mathrm{g}$ and $30 \mathrm{~m}^{2} / \mathrm{g}$ for increasing the PEI ratios from $30 \mathrm{wt} \%$ up to 50-60 wt.\%, as PEI blocked the existing deep micropores in BP-2000 and EC 600JD. While steam treated activated commercial carbon exhibited the highest $\mathrm{CO}_{2}$ adsorption capacity among polymer-free carbons, $\sim 126 \mathrm{mgCO}_{2} / \mathrm{g}$ sorbent, the adsorption capacity of BP-2000 with 50 wt.\% PEI (SSA $\sim 37 \mathrm{~m}^{2} / \mathrm{g}$ ) reached $135 \mathrm{mgCO}_{2} / \mathrm{g}$ sorbent. The efficiency of this sorbent reached $0.26 \mathrm{mmolCO}_{2} / \mathrm{mol}$ of amine groups from PEI. Further increasing the PEI ratios resulted in lower efficiency. Despite the negligible microporosity of these nanocomposites, the majority of nitrogen functional groups of PEI were located on the external surfaces of the CB particles. PEI loadings exceeding $60 \mathrm{wt} . \%$ resulted in external aggregates with limited $\mathrm{CO}_{2}$ accessibility. Studies on the textural porosity of this type of nanocomposites and its impacts on gas diffusion are still missing and could lead to sorbents with improved surface distribution of amine sites and consequently superior gravimetric capacity.

Alternatively to non-covalent functionalization of $\mathrm{CB}$, surface covalent grafting of hyperbranched polymers having quaternary ammonium cations and hydroxyl counter ions onto $\mathrm{CB}$ via atom transfer radical polymerization (ATRP) are attractive for reversible $\mathrm{CO}_{2}$ capture [166, 313]. In fact, these types of polymer surface groups have been found to reversibly adsorb $\mathrm{CO}_{2}$ using humidity swing at very fast rates, at least an order of magnitude faster than commercial sorbent resins containing amine groups. And fast sorption kinetics is of major importance for sorbent regeneration in less time and with less energy in industrial scale carbon capture [313]. At low humidity conditions, the CB-polymer nanocomposites bind to $\mathrm{CO}_{2}$, whereas at high humidity levels, the carbonate and bicarbonate species react with water and release $\mathrm{CO}_{2}$. This mechanism mimics the functioning of plant leaves, except that humidity, rather than light, regulates the sorbent's "breathing". Compared to the same polymer having pores templated by colloidal silica crystal or by emulsion, the $\mathrm{CB}$ nanocomposites display similar adsorption and desorption rates, and CB-composite properties could be further improved by maximizing the surface area and porosity. In addition, such polymer grafting method with electrostatically charged groups improved alkali resistance of $\mathrm{CB}$, consequently prolonging the life of the sorbents and retaining their reactivity upon cycling [133, 207, 260]. These coatings further lead to higher water solubility of functional $\mathrm{CB}$ in aqueous and other polar solvents [267-269], and enhanced 
thermochemical stability. These properties make CB-polymer nanocomposites of major importance for large-scale carbon capture using photochemical energy, as previously described for functional nanofluids [166, 295, 296].

\section{Conclusions}

The structure and chemistry of Carbon Black (CB) largely resemble that of most graphitic and turbostratic carbons. The existing link is the extended two-dimensional $\mathrm{sp}^{2} \mathrm{C}$ lattice of the BSU and of most carbon materials. Surface heterogeneities such as lattice defects and small size of graphene units forming each BSU give curvature and favor stacking mismatches, further resembling a vast array of porous carbon materials. As discussed in this manuscript, $\mathrm{CB}$ comprises a family of nanostructured materials that are non-graphitizable and that are mixed with amorphous carbon, although small localized graphitic ordering has been reported for few examples of CB. The resulting morphology and particle sizes depend on the preparation method and precursors used. CB is prepared in a controlled manner, making it different from other pyrolysis carbonaceous products, i.e. Black Carbon (BC) and Soot. This review is a comprehensive report of $\mathrm{CB}$ uses in renewable energy and environmental applications, with a critical analysis of the fundamental properties of $\mathrm{CB}$ essential to these applications. The CB structure and physicochemical properties are covered in terms of pioneering and recent chemical modifications, in particular those relevant to technologically emerging applications in energy storage and conversion processes. $\mathrm{CB}$ is widely available, it is inexpensive compared to most synthetic carbons, and it is an excellent platform for the preparation of carbon-based composites for many applications. The highlights of this review are expected to engross the attention of not only the chemistry community, but also chemical engineers and materials/environmental scientists who are dealing with renewable energy challenges.

With CB being one of the most abundantly produced forms of carbon and that approximately $70 \%$ of all CB produced globally is used as pigment and as reinforcing phase in tires, there is a need to highlight recent scientific reports on other uses of CB that are of current interest, such as renewable energy harvesting and environmental remediation. Compared to other carbon nanomaterials, $\mathrm{CB}$ and functional $\mathrm{CB}$ materials have proven competitive in terms of properties for $\mathrm{CO}_{2}$ separations, supercapacitors, batteries, and as catalyst supports in fuel cells and solar cells. Some key feature of CB composites was the ability to tailor the surface 
functionality, and/or porosity from subnanometer pores up to the mesoscale. Moreover, the unique structure of $\mathrm{CB}$ makes it an excellent starting material for graphene quantum dots, hollow carbon spheres, and as components in "brick and mortar" carbon-carbon nanocomposites with unique electrical conductivity properties and reactivity. Those include advanced chemical methods to modify the surfaces such as ATRP and other polymer grafting reactions mainly due to delocalized electrons existing in the $\mathrm{CB}$ structure, with their many possibilities to prepare functional nanocomposites.

Future research may reveal other interesting properties of $\mathrm{CB}$-based materials and $\mathrm{CB}$ chemistry. For instance, although wet oxidation strategies are applicable for all CB grades, many questions still remain open for investigation, especially the existing differences among wet oxidation methods under conventional heating and microwave irradiation. Such differences determine whether oxidized CB, hollow spheres or graphene nanosheets form. Microwave [314] and ultrasound [315] are potential substitutes for conventional energyintensive processing methods, also of interest for selectively peeling-off specific fragments from the $\mathrm{CB}$ surfaces without the need for oxidation. As an example of graphite exfoliation in organic solvents [316-318], surfactants and polymers [319, 320], and RTIL [321] in ultrasound, similar methods can be investigated for CB. Besides graphene sheets and GQD, isolating fullerene-like fragments could shed new light on the structure of the different $\mathrm{CB}$ grades, their reactivity and electrical properties. HRTEM, atomic force microscopy (AFM), scanning tunneling microscopy (STM) and other high-resolution surface techniques help identify such fragments, study topology and probe for pentagonal and heptagonal defects on those various fragments. Centrifuge methods can further be developed to separate all possible products, as it has been done for graphene produced from liquid exfoliation of graphite. In addition to those, the abundance of $\mathrm{CB}$ with distinct surface reactivity, electrical conductivity, particle and aggregate sizes have yet to be systematically compared, as these offer almost unlimited possibilities to the design functional carbon materials with unique properties for energy and environmental applications.

Renewable energy applications predominantly involve the transfer and storage of charged species, hence electrical and ion conductivities are essential. CB is electrically conducting; it is also possible to alter its porosity and surface chemistry to best tailor its properties to the application. Higher electrical conductivity is often sought, although a compromise is made when surface functionalization (which is known to impact negatively on electrical conductivity) can improve performance, stability, and durability of the device. Higher surface 
areas are also important, but this has to be balanced since deep microporosity can at times be detrimental. In the following, we have recapped all key aspects of CB properties adapted and altered to meet the specific needs of key renewable energy applications: fuel cells, batteries, supercapacitors, and solar devices.

In fuel cells, $\mathrm{CB}$ has been applied as conductive support for catalysts (mainly Pt nanoparticles) where the interplay of surface area, porosity, and surface functional groups determine the resulting performance of the electrode. Porosity and functional groups prevent the aggregation of catalyst particles, but nanoparticles trapped in deep microporosity cannot contact the proton exchange membrane (mainly Nafion) impacting negatively on performance. Besides selecting CB with limited or no microporosity, a strategy to avoid this issue is the synthesis of nanoparticles with geometries and sizes that impede entrapment in micropores. Nanoparticle dispersion and stability can also be improved using alloy catalysts (e.g. Pt-Ru) able to bind more strongly to $\mathrm{CB}$. Alloying of Pd with boron (borane dimethylamine) has also been shown to improve nanoparticle stability on CB. Pre-treatment of $\mathrm{CB}$ such as oxidation can change the nature and number of the surface functional group allowing the tuning of nanoparticle stability. Oxidation can also introduce ketone functional groups able to catalyse water and alcohol oxidation reactions. However, oxygen-containing groups can also make $\mathrm{CB}$ hydrophilic impacting on the water removal necessary for the operation of the cell. For this reason, CB for ORR is commonly thermally treated in an inert atmosphere (graphitized) to remove oxygen functionalities and increase hydrophobicity. Graphitized CB has been shown to increase the catalyst tolerance towards carbon monoxide. Boron and nitrogen-doped CB has intrinsic catalytic ORR activity, B and N-doped CB were prepared mechanochemically in the presence of boron nitride, for example. F-doping has also been reported to enhance ORR. CB-polymer composites (with polyethyleneimine) have been used to promote nanoparticle dispersion and accessibility. Other aspects to consider in using $\mathrm{CB}$ as catalyst support are the limited stability in acid and alkaline media, and the easy of oxidation at relatively low potentials $(0.9 \mathrm{~V}$ vs SHE) affecting surface area, porosity and surface chemistry. CB can also be used in gas diffusion layers (GDLs), published work shows how gas permeability, electrical conductivity, hydrophobicity (water removal), and mechanical stability can be improved and tuned when using blends of different CB grades in the GDLs, or when conducting polymers are used.

In batteries, $\mathrm{CB}$ has been applied for both cathodes and anodes. CB improves the electrical conductivity of the electrodes when electrically insulating polymeric binders are used. 
Cathode composites of $\mathrm{CB}$ with $\mathrm{MnO}_{2}, \mathrm{LiMn}_{2} \mathrm{O}_{2}$-spinel, $\mathrm{LiCoO}_{2}$, or $\mathrm{LiFePO}_{2}$ have been reported for lithium-ion batteries. Functionalised CB has been used in lithium-sulfur batteries achieving high reversible specific charge capacity above $1000 \mathrm{mAh} / \mathrm{g}$ using CB with $\mathrm{N}$ sites added via $\mathrm{NH}_{3}$ activation. Partially exfoliated $\mathrm{CB}$ via high energy ball milling was also added to sulfur with resulting capacities of about $800 \mathrm{mAh} / \mathrm{g}$ after 200 cycles. Direct ball milling of CB with S produced sulfur-coated CB applied in Li-S batteries with capacities exceeding $1000 \mathrm{mAh} / \mathrm{g}$. The CB-S particles were stabilised with a carbon coating to prevent the dissolution of polysulfides. Anodes for Li-ion batteries were made from the graphitisation of spray-dried CB-sucrose mixtures, the performance of the resulting electrode was superior to that made from commercial graphite microspheres. Other LIB anodes were made from CB and $\mathrm{ZnO}$ (from atomic layer deposition) attaining specific capacities superior to the theoretical value of $\mathrm{ZnO}(987 \mathrm{mAh} / \mathrm{g})$. Strong $\mathrm{ZnO}-\mathrm{CB}$ interactions prevented large volume changes of $\mathrm{ZnO}$ during lithium intercalation and deintercalation. The addition of a few weight percent of $\mathrm{CB}$ to $\mathrm{ZnO}$ has also been shown to improve the performance of $\mathrm{Li}$-air batteries. CB materials have been used in sodium-ion batteries achieving a value of reversible capacity of $200 \mathrm{mAh} / \mathrm{g}$ thanks to the low density and disordered carbon sheets stacking of $\mathrm{CB}$ helping the reversibility of the process. Silicon can intercalate a large number of lithium ions but with a correspondingly large volume change (swelling), in the attempt to solve this problem silicon has been deposited in the interparticle space of $\mathrm{CB}$ aggregates improving durability and cycling life of silicon-based anodes.

In supercapacitors, $\mathrm{CB}$ alone was shown to achieve limited gravimetric capacities of a few F/g. This could increase via oxidation introducing electroactive species contributing to pseudocapacitive charge storage. More importantly, CB was successfully used in carboncarbon composites to increase the capacitance of reduced graphene oxide (rGO) from $69 \mathrm{~F} / \mathrm{g}$ for $\mathrm{rGO}$ to $175 \mathrm{~F} / \mathrm{g}$ for $\mathrm{rGO}-\mathrm{CB}$ in aqueous $\mathrm{KOH}$ electrolyte. This was related to the intimate mixing of $\mathrm{rGO}$ and $\mathrm{CB}$ resulting in the formation of interlayer mesopores increasing significantly the specific surface area of the blended materials. CB acted as pillars for the layered rGO lattice. Decreased electrolyte diffusion resistance was also observed in rGO-CB fibers using $\mathrm{H}_{3} \mathrm{PO}_{4}$ electrolyte in PVA, again thanks to the presence of mesoporosity. CBpillared graphene composites have also been used in electrochemical flow capacitors where flowable carbon-electrolyte slurries are used to store energy. Here, the particle porosity of CB-graphene composites was maintained even when stacking of layered particles in a slurry took place. In a different approach, CB was integrated is an ordered mesoporous carbon 
(OMC) network. Thanks to the presence of $\mathrm{CB}$ during the preparation of the material, the resulting OMC-CB achieved high capacitance values comparable to those of carbon mesoporous carbons with a similar specific surface area. CB can also be combined with conducting polymers. Capacitance values for neat polypyrrole (PPY) nanoparticles in aqueous $\mathrm{NaNO}_{3}$ electrolyte was $\sim 120 \mathrm{~F} / \mathrm{g}$, which increased to $366 \mathrm{~F} / \mathrm{g}$ for composite with 20 wt.\% CB (core-shell CB-PPY).

In solar devices, $\mathrm{CB}$ has been applied to improve the performance of photocatalysts and thermal energy absorption. In dye-sensitized solar cells, CB has been shown to decrease electron-hole recombination in the semiconducting oxide, typically $\mathrm{TiO}_{2}$, and improve its thermal stability, preventing anatase to rutile transition. $\mathrm{CB}$ added to $\mathrm{TiO}_{2}$ was $50 \%$ more active than $\mathrm{TiO}_{2}$ film alone. Key to this improvement was the method of preparation of CB$\mathrm{TiO}_{2}$ composites. Sol-gel mixtures resulted in better and more homogenously distributed contact between $\mathrm{CB}$ and $\mathrm{TiO}_{2}$, which could not be achieved via physical mixing. CB composites have also been evaluated as alternatives to Pt on transparent counter-electrodes (FTO, ITO) to facilitate the reduction of $\mathrm{I}_{3}$. Cell efficiencies above 9\% were achieved using $\mathrm{CB}$ in place of $\mathrm{Pt}$, the activity was correlated to a large number of edge sites of $\mathrm{CB}$ short graphene domains. $\mathrm{CB}$ was also used as a precursor of graphene quantum dots (GQD) obtained via oxidation and subsequent exfoliation. GQD have prolonged quantum confinement effects, stable photoluminescence and excellent chemical stability, ideal properties for photonic devices including solar cells. GQD-coated carbon aerogel composites cast on FTO glass performed better than Pt-FTO. N-doped GQD have been proposed as efficient $\mathrm{I}_{3}$ reduction photocatalysts thus there is the opportunity to prepare N-doped GQD as a cheaper alternative to Pt. Pt-free counter electrodes were also made from CB compounded in epoxy resins then coated on stainless steel, the CB-epoxy resin functioned also as a corrosion protection layer. Different from photovoltaics, yet another interesting application of $\mathrm{CB}$ is in $\mathrm{C}$-based nanofluids working on the principle of solar energy absorption. $\mathrm{CB}$ nanofluids and water have a nearly perfect infrared absorption, and CB particle networking improves the thermal conductivity of the fluid. Such fluids are of great interest for the development of solar heaters and distillation systems which are capable of generating vapor without the requirement of heating the fluid volume. We hope this final recount have now fully motivated the reader to further investigate the many different applications of $\mathrm{CB}$, and accordingly find the most suitable ways to apply this economical and largely available carbon materials in the field of renewable energy. 


\section{Abbreviation List}

AB: Acetylene black

AFC: alkaline fuel cell

AFM: atomic force microscopy

ALD: atomic layer deposition

AOR: alcohol oxidation reaction

ASA: active surface area

ATRP: atom transfer radical polymerization

bpy: 2,2-Bipyridine

BET: Brunauer-Emmett-Teller

BSU: basic structural unit

CAs: carbon aerogels

CB: Carbon Black

CBNPs: carbon black nanoparticles

CNT: carbon nanotube

CCS: carbon capture and sequestration

$\mathrm{CV}$ : cyclic voltammogram

DCC: dicyclohexylcarbodiimide

DETA: diethylenetriamine

DFAFC: direct formic acid fuel cell

DMAP: 4-Dimethylaminopyridine

DMC: dimethyl carbonate

DMF: dimethylformamide

DMSO: dimethyl Sulfoxide

DMTMM: 4-(4,6-dimethoxy-1,3,5-triazin-2-yl)-4-methylmorpholinium chloride

DMFC: direct methanol fuel cell

dNbpy: 4,4'-dinonyl-2,2'-dipyridyl

DSSC: dye sensitized solar cell

EC: ethylene carbonate

EDCI: 1-ethyl-3-(3-dimethylaminopropyl) carbodiimide

EDLC: electrical double layer capacitor

EFC: electrochemical flow capacitor 
EDL: electrical double layer

$e^{-}:$electron

ESR: electrochemical series resistance

EV: electric vehicle

FB: Furnace Black

FBO: oxidized Furnace Black

FESEM: field emission scanning electron microscopy

FTO: fluoride-doped tin oxide

GDC: galvanostatic charge-discharge

GDE: gas diffusion electrode

GDL: gas diffusion layer

GQD: graphene quantum dot

GM: graphite microsphere

GO: graphene oxide

$h^{+}:$hole

HACA: hydrogen-abstraction/carbon-addition

HRTEM: high resolution transmission electron microscopy

IARC: International Agency for Research on Cancer

ITO: indium tin oxide

$\mathrm{J}_{\mathrm{SC}}$ : short circuit current

LIB: lithium-ion battery

LSV: linear sweep voltammetry

MBS: molecular basket sorbent

MEA: monoethanolamine

MWNT: multiwalled carbon nanotube

n-BA: n-butyl acrylate

ND: nanodiamond

NIB: sodium-ion battery

Ni-MH: nickel metal hydride

NMP: N-methyl-2-pyrrolidone

NOx: nitrogen oxides

NP: nanoparticle

OC: oxygen carrier 
OCB: oxidized carbon black

ODA: octadecyl amine

OLC: onion-like carbon

OMC: ordered mesoporous carbon

ORR: oxygen reduction reaction

PAFC: phosphoric acid fuel cell

PAH: polycyclic aromatic hydrocarbon

PCE: photocatalytic efficiency

PEMFC: proton exchange membrane fuel cell

PEI: polyethyleneimine

PEO: poly(ethylene oxide) polymer

PEO-PPO-PEO: poly-ethylene oxide-poly-propylene oxide-poly-ethylene oxide

PHMAS: poly(2-hydroxy-3-(methacryloyloxy) propane-1-sulfonate)

PMMA: poly(methyl methacrylate)

PO: pyrolysis oil

PPY: polypyrrole

PPS: primary particle size

PVA: polyvinyl alcohol

PVDF: poly(vinylidene fluoride)

PVDF-HFP: poly(vinylidene difluoride-co-hexafluoropropylene

PMDETA: $\mathrm{N}, \mathrm{N}, \mathrm{N}^{\prime}, \mathrm{N}^{\prime \prime}, \mathrm{N}^{\prime \prime}-$ Pentamethyldiethylenetriamine

PDMAEMA: poly(2-dimethylaminoethyl methacrylate)

PSD: pore size distribution

PSt-TEMPO: (TEMPO)-terminated polystyrene

rGO: reduced graphene oxide

RTIL: room temperature ionic liquid

SEI: solid electrolyte interphase

SEM: scanning electron microscopy

SHE: standard hydrogen electrode

SOFC: solid oxide fuel cell

SOx: sulfur oxides

SS: stainless steel

SSA: specific surface area 
STM: scanning tunneling microscopy

STSA: statistical film thickness surface area

SW: Stone-Wales

SWNT: single-wall carbon nanotube

TEA: triethylamine

TEM: transmission electron microscopy

TEMPO: 2,2,6,6-tetramethyl-1-piperidinyloxy

THF: tetrahydrofuran

TSIL: task specific ionic liquid

$\mathrm{V}_{\mathrm{OC}}$ : open circuit potential

WOR: water oxidation reaction

\section{Acknowledgments}

EA wish to acknowledge the Reducing Industrial Carbon Emission (RICE) research and innovation project funded by the Welsh European Funding Office (WEFO), the European Regional Development Fund (ERDF), through the Welsh Government. SK and EA also wish to thank the Engineering and Physical Sciences Research Council-Impact Acceleration Account (EPSRC-IAA) for providing funding to project code RIF67. SK wish to acknowledge funding from the European Union's Horizon 2020 research and innovation program under the Marie Skłodowska- Curie grant agreement No 663830.

\section{References}

[1] Ceresana, Market Study: Carbon Black (3rd edition), 2018. https://www.ceresana.com/en/market-studies/chemicals/carbon-black/. (Accessed September 2018).

[2] M.a. Markets, Carbon Nanotubes (CNT) Market by Type (Single, Multi Walled), Method (Chemical Vapor Deposition, Catalytic Chemical Vapor Deposition, High Pressure Carbon Monoxide), Application (Electronics, Chemical, Batteries, Energy, Medical) - Global Forecast to 2022, 2017. https://www.marketsandmarkets.com/PressReleases/carbonnanotubes.asp. (Accessed September 2018).

[3] Cheaptubes.com, Multi Walled Carbon Nanotubes, 2018. https://www.cheaptubes.com/product-category/multi-walled-carbon-nanotubes/. (Accessed September 2018).

[4] Cheaptubes.com, Graphene Nanoplatelets, 2018.

https://www.cheaptubes.com/product/graphene-nanoplatelets/. (Accessed September 2018). [5] Cheaptubes.com, Carbon Fullerenes C60, 2018. https://www.cheaptubes.com/product/carbon-fullerenes-c60/ (Accessed September 2018). [6] Carbon, 6. Carbon Black, Ullmann's Encyclopedia of Industrial Chemistry. 
[7] Carbon Black, Encyclopedia of Polymer Science and Technology.

[8] I.W.G.o.t.E.o.C.R.t. Humans, Carbon black, titanium dioxide, and talc, IARC Monogr

Eval Carcinog Risks Hum 93 (2010) 1-413.

[9] COKE, CARBON BLACK, AND GRAPHITE, Handbook of Petroleum Product

Analysis.

[10] H.P. Boehm, Some aspects of the surface chemistry of carbon blacks and other carbons, Carbon 32(5) (1994) 759-769.

[11] A.A. Deshmukh, S.D. Mhlanga, N.J. Coville, Carbon spheres, Materials Science and Engineering: R: Reports 70(1) (2010) 1-28.

[12] M. Ozawa, E. Ōsawa, Chapter 6 - Carbon blacks as the source materials for carbon nanotechnology, in: L. Dai (Ed.), Carbon Nanotechnology, Elsevier, Amsterdam, 2006, pp. 127-151.

[13] H. Marsh, F. Rodríguez-Reinoso, CHAPTER 2 - Activated Carbon (Origins), in: H. Marsh, F. Rodríguez-Reinoso (Eds.), Activated Carbon, Elsevier Science Ltd, Oxford, 2006, pp. 13-86.

[14] R.C.B. Jean-Baptiste Donnet, Meng Jiao Wang, Carbon Black: Science and Technology, Second edition ed., Marcel Dekker, New York, 1993.

[15] M. Pawlyta, J.-N. Rouzaud, S. Duber, Raman microspectroscopy characterization of carbon blacks: Spectral analysis and structural information, Carbon 84 (2015) 479-490.

[16] J.P. Abrahamson, M. Singh, J.P. Mathews, R.L. Vander Wal, Pulsed laser annealing of carbon black, Carbon 124 (2017) 380-390.

[17] S. Ban, K. Malek, C. Huang, Z. Liu, A molecular model for carbon black primary particles with internal nanoporosity, Carbon 49(10) (2011) 3362-3370.

[18] P.M. Wilson, F. Orange, M.J.F. Guinel, M. Shekhirev, Y. Gao, J.A. Colon Santana, A.A. Gusev, P.A. Dowben, Y. Lu, A. Sinitskii, Oxidative peeling of carbon black nanoparticles, RSC Advances 5(112) (2015) 92539-92544.

[19] K. Jurkiewicz, M. Pawlyta, A. Burian, Structure of Carbon Materials Explored by Local Transmission Electron Microscopy and Global Powder Diffraction Probes, C 4(4) (2018) 68.

[20] B. Apicella, P. Pré, M. Alfè, A. Ciajolo, V. Gargiulo, C. Russo, A. Tregrossi, D.

Deldique, J.N. Rouzaud, Soot nanostructure evolution in premixed flames by High

Resolution Electron Transmission Microscopy (HRTEM), Proceedings of the Combustion Institute 35(2) (2015) 1895-1902.

[21] H.W. Kroto, K. McKay, The formation of quasi-icosahedral spiral shell carbon particles, Nature 331(6154) (1988) 328-331.

[22] L.B. Ebert, The icospiral model of combustion soot, Carbon 31(1) (1993) 239-240.

[23] J. Guo, J.R. Morris, Y. Ihm, C.I. Contescu, N.C. Gallego, G. Duscher, S.J. Pennycook, M.F. Chisholm, Topological Defects: Origin of Nanopores and Enhanced Adsorption Performance in Nanoporous Carbon, Small 8(21) (2012) 3283-3288.

[24] P. Gerhardt, S. Löffler, K.H. Homann, Polyhedral carbon ions in hydrocarbon flames, Chem. Phys. Lett. 137(4) (1987) 306-310.

[25] S. Iijima, Direct observation of the tetrahedral bonding in graphitized carbon black by high resolution electron microscopy, Journal of Crystal Growth 50(3) (1980) 675-683.

[26] P.J.F. Harris, Structure of non-graphitising carbons, International Materials Reviews 42(5) (1997) 206-218.

[27] P.R. Buseck, K. Adachi, A. Gelencsér, É. Tompa, M. Pósfai, Ns-Soot: A Material-Based Term for Strongly Light-Absorbing Carbonaceous Particles, Aerosol Science and Technology 48(7) (2014) 777-788.

[28] P. Kim, Across the border, Nature Materials 9 (2010) 792. 
[29] F. Cataldo, The impact of a fullerene-like concept in carbon black science, Carbon 40(2) (2002) 157-162.

[30] Y.G. Kryazhev, N.N. Koval', V.A. Likholobov, A.D. Teresov, V.A. Drozdov, M.V.

Trenikhin, Structural transformations in nanoglobular carbon under the action of pulsed highenergy-density electron beam, Technical Physics Letters 38(4) (2012) 301-303.

[31] M.V. Trenikhin, Y.G. Kryazhev, O.V. Protasova, I.V. Muromtsev, V.A. Drozdov, V.A. Likholobov, A.D. Teresov, N.N. Koval', G.M. Seropyan, Structural Transformations in Carbon Black Under the Action of Powerful Energy Beams, Springer International Publishing, Cham, 2014, pp. 159-164.

[32] M.V. Trenikhin, O.V. Ivashchenko, V.S. Eliseev, B.P. Tolochko, A.B. Arbuzov, I.V. Muromtsev, Y.G. Kryazhev, V.A. Drozdov, E. Sazhina, V.A. Likholobov, Electron Microscopy Investigation of Structural Transformation of Carbon Black under Influence of High-Energy Electron Beam, Fullerenes, Nanotubes and Carbon Nanostructures 23(9) (2015) 801-806.

[33] O.V. Ivashchenko, M.V. Trenikhin, Y.G. Kryazhev, B.P. Tolochko, V.S. Eliseev, A.B. Arbuzov, V.A. Drozdov, V.A. Likholobov, Structural transformations of carbon black by high-energy laser and electron irradiation, Nanotechnologies in Russia 10(9) (2015) 696-700. [34] M.C. Evora, D. Klosterman, K. Lafdi, L. Li, J.L. Abot, Functionalization of carbon nanofibers through electron beam irradiation, Carbon 48(7) (2010) 2037-2046.

[35] N.S. Xu, R.V. Latham, 5 - Electron Emission Based Breakdown Mechanisms, in: R.V. Latham (Ed.), High Voltage Vacuum Insulation, Academic Press, London, 1995, pp. 165204.

[36] O.V. Yazyev, I. Tavernelli, U. Rothlisberger, L. Helm, Early stages of radiation damage in graphite and carbon nanostructures: A first-principles molecular dynamics study, Physical Review B 75(11) (2007) 115418.

[37] A.J. Stone, D.J. Wales, Theoretical studies of icosahedral C60 and some related species, Chem. Phys. Lett. 128(5-6) (1986) 501-503.

[38] H. Zou, Y. Yang, Q. Li, J. Zhang, Z. Liu, X. Guo, Z. Du, Electron beam-induced structure transformation of single-walled carbon nanotubes, Carbon 40(12) (2002) 22822284.

[39] B. Peng, M. Locascio, P. Zapol, S. Li, S.L. Mielke, G.C. Schatz, H.D. Espinosa, Measurements of near-ultimate strength for multiwalled carbon nanotubes and irradiationinduced crosslinking improvements, Nature nanotechnology 3(10) (2008) 626. [40] C.P. Ewels, R.H. Telling, A.A. El-Barbary, M.I. Heggie, P.R. Briddon, Metastable Frenkel Pair Defect in Graphite: Source of Wigner Energy?, Physical Review Letters 91(2) (2003) 025505.

[41] V. Asokan, V. Venkatachalapathy, K. Rajavel, D.N. Madsen, Microwave irradiation on carbon black: Studies on the transformation of particles into nano-balls, nano-sticks and nano-onion like structures, Journal of Physics and Chemistry of Solids 99 (2016) 173-181. [42] J. Wutthiprom, N. Phattharasupakun, M. Sawangphruk, Turning Carbon Black to Hollow Carbon Nanospheres for Enhancing Charge Storage Capacities of LiMn2O4, LiCoO2, LiNiMnCoO2, and LiFePO4 Lithium-Ion Batteries, ACS Omega 2(7) (2017) 37303738 .

[43] Y.G. Andreev, P.G. Bruce, Size and shape of graphene layers in commercial carbon blacks established by Debye refinement, Journal of Applied Crystallography 49(1) (2016) 2430.

[44] Q. Li, X. Zhang, G. Wu, S. Xu, C. Wu, Sonochemical preparation of carbon nanosheet from carbon black, Ultrasonics Sonochemistry 14(2) (2007) 225-228. 
[45] P. Xue, J. Gao, Y. Bao, J. Wang, Q. Li, C. Wu, An analysis of microstructural variations in carbon black modified by oxidation or ultrasound, Carbon 49(10) (2011) 3346-3355.

[46] Y. Gao, Y.S. Zhou, J.B. Park, H. Wang, X.N. He, H.F. Luo, L. Jiang, Y.F. Lu, Resonant excitation of precursor molecules in improving the particle crystallinity, growth rate and optical limiting performance of carbon nano-onions, Nanotechnology 22(16) (2011) 165604. [47] Standard Test Method for Carbon Black\&\#x2014; Total and External Surface Area by Nitrogen Adsorption.

[48] M. Kruk, M. Jaroniec, Gas Adsorption Characterization of Ordered Organic-Inorganic Nanocomposite Materials, Chemistry of Materials 13(10) (2001) 3169-3183.

[49] J. Rouquerol, D. Avnir, C.W. Fairbridge, D.H. Everett, J.H. Haynes, N. Pernicone, J.D.F. Ramsay, K.S.W. Sing, K.K. Unger, RECOMMENDATIONS FOR THE CHARACTERIZATION OF POROUS SOLIDS, Pure Appl. Chem. 66(8) (1994) 1739-1758. [50] S. Brunauer, P.H. Emmett, E. Teller, Adsorption of Gases in Multimolecular Layers, Journal of the American Chemical Society 60(2) (1938) 309-319.

[51] M. Kruk, M. Jaroniec, Y. Bereznitski, Adsorption Study of Porous Structure

Development in Carbon Blacks, Journal of Colloid and Interface Science 182(1) (1996) 282288.

[52] M. Kruk, M. Jaroniec, K.P. Gadkaree, Nitrogen Adsorption Studies of Novel Synthetic Active Carbons, Journal of Colloid and Interface Science 192(1) (1997) 250-256.

[53] J. Rouquerol, P. Llewellyn, F. Rouquerol, Is the bet equation applicable to microporous adsorbents?, in: P.L. Llewellyn, F. Rodriquez-Reinoso, J. Rouqerol, N. Seaton (Eds.), Studies in Surface Science and Catalysis, Elsevier2007, pp. 49-56.

[54] J. Choma, M. Jaroniec, Determination of the Specific Surface Areas of Non-Porous and Macroporous Carbons, Adsorption Science \& Technology 19(9) (2001) 765-776.

[55] J. Choma, J. Gorka, M. Jaroniec, Mesoporous carbons synthesized by soft-templating method: Determination of pore size distribution from argon and nitrogen adsorption isotherms, Microporous and Mesoporous Materials 112(1-3) (2008) 573-579.

[56] B.C. Lippens, J.H. de Boer, Studies on pore systems in catalysts: V. The t method, Journal of Catalysis 4(3) (1965) 319-323.

[57] M. Jaroniec, K. Kaneko, Physicochemical Foundations for Characterization of Adsorbents by Using High-Resolution Comparative Plots, Langmuir 13(24) (1997) 65896596.

[58] L. Gardner, M. Kruk, M. Jaroniec, Reference Data for Argon Adsorption on Graphitized and Nongraphitized Carbon Blacks, The Journal of Physical Chemistry B 105(50) (2001) 12516-12523.

[59] R.H. Bradley, I. Sutherland, E. Sheng, Carbon Surface: Area, Porosity, Chemistry, and Energy, Journal of Colloid and Interface Science 179(2) (1996) 561-569.

[60] K. Kawasumi, Q. Zhang, Y. Segawa, L.T. Scott, K. Itami, A grossly warped nanographene and the consequences of multiple odd-membered-ring defects, Nature Chemistry 5 (2013) 739.

[61] D. Tashima, H. Yoshitama, M. Otsubo, S. Maeno, Y. Nagasawa, Evaluation of electric double layer capacitor using Ketjenblack as conductive nanofiller, Electrochimica Acta 56(24) (2011) 8941-8946.

[62] D. Liu, L. Ji, Y. Ding, X. Weng, F. Yang, X. Zhang, Mesoporous carbon black as a metal-free electrocatalyst for highly effective determination of chromium(VI), Journal of Electroanalytical Chemistry 803 (2017) 58-64.

[63] N.V. Maltseva, V.A. Golovin, Y.O. Chikunova, E.N. Gribov, The Effect of Electrochemical and Gas Phase Activation of High Surface Area Carbon Black Ketjen Black 
EC 600 DJ on Its Surface Composition, Electrochemical Capacitance, and Stability, Russian Journal of Electrochemistry 54(5) (2018) 426-432.

[64] D. Tashima, T. Kishita, S. Maeno, Y. Nagasawa, Mesoporous graphitized Ketjenblack as conductive nanofiller for supercapacitors, Materials Letters 110 (2013) 105-107.

[65] A.G. Pandolfo, A.F. Hollenkamp, Carbon properties and their role in supercapacitors, Journal of Power Sources 157(1) (2006) 11-27.

[66] K. Ono, M. Yanaka, Y. Saito, H. Aoki, O. Fukuda, T. Aoki, T. Yamaguchi, Effect of benzene-acetylene compositions on carbon black configurations produced by benzene pyrolysis, Chemical Engineering Journal 215-216 (2013) 128-135.

[67] K. Ono, A. Watanabe, K. Dewa, Y. Matsukawa, Y. Saito, Y. Matsushita, H. Aoki, O. Fukuda, T. Aoki, T. Yamaguchi, Detailed kinetic analysis of the impact of nucleation behavior and particle size distribution on the configurations of carbon black, Journal of Nanoparticle Research 16(7) (2014) 2519.

[68] K. Ono, A. Watanabe, K. Dewa, Y. Matsukawa, Y. Saito, Y. Matsushita, H. Aoki, O. Fukuda, T. Aoki, T. Yamaguchi, Detailed kinetic analysis of the effect of benzene-acetylene composition on the configuration of carbon nanoparticles, Chemical Engineering Journal 250 (2014) 66-75.

[69] K. Ono, K. Dewa, Y. Matsukawa, Y. Saito, Y. Matsushita, H. Aoki, K. Era, T. Aoki, T. Yamaguchi, Experimental evidence for the sintering of primary soot particles, Journal of Aerosol Science 105 (2017) 1-9.

[70] K. Dewa, K. Ono, A. Watanabe, K. Takahashi, Y. Matsukawa, Y. Saito, Y. Matsushita, H. Aoki, K. Era, T. Aoki, T. Yamaguchi, Evolution of size distribution and morphology of carbon nanoparticles during ethylene pyrolysis, Combustion and Flame 163 (2016) 115-121. [71] K. Ono, M. Yanaka, S. Tanaka, Y. Saito, H. Aoki, O. Fukuda, T. Aoki, T. Yamaguchi, Influence of furnace temperature and residence time on configurations of carbon black, Chemical Engineering Journal 200-202 (2012) 541-548.

[72] W. Zhu, D.E. Miser, W. Geoffrey Chan, M.R. Hajaligol, HRTEM investigation of some commercially available furnace carbon blacks, Carbon 42(8) (2004) 1841-1845.

[73] K. Mukawa, N. Oyama, H. Ando, T. Sugiyama, S. Ogo, Y. Sekine, Synthesis of stable anisotropic carbon particle aggregates covered by surface nano-graphitic sheets, Carbon 88 (2015) 33-41.

[74] E.A. Grulke, S.B. Rice, J. Xiong, K. Yamamoto, T.H. Yoon, K. Thomson, M.

Saffaripour, G.J. Smallwood, J.W. Lambert, A.J. Stromberg, R. Macy, N.J. Briot, D. Qian, Size and shape distributions of carbon black aggregates by transmission electron microscopy, Carbon 130 (2018) 822-833.

[75] S. Mohsenian, M.S. Esmaili, J. Fathi, B. Shokri, Hydrogen and carbon black nanospheres production via thermal plasma pyrolysis of polymers, International Journal of Hydrogen Energy 41(38) (2016) 16656-16663.

[76] N.J. Hardman, The new carbon black and its role in the United States manufacturing renaissance, Reinforced Plastics 61(3) (2017) 145-148.

[77] M. Gautier, V. Rohani, L. Fulcheri, J.P. Trelles, Influence of temperature and pressure on carbon black size distribution during allothermal cracking of methane, Aerosol Science and Technology 50(1) (2016) 26-40.

[78] M. Gautier, V. Rohani, L. Fulcheri, Direct decarbonization of methane by thermal plasma for the production of hydrogen and high value-added carbon black, International Journal of Hydrogen Energy 42(47) (2017) 28140-28156.

[79] L.F. Frédéric Fabry, Synthesis of Carbon Blacks from HDPE plastic by 3-phase AC thermal plasma, 23rd International Symposium on Plasma Chemistry, Montréal, Canada, 2017. 
[80] F. Fabry, B. Ravary, P. Bertrand, J.M. Badie, L. Fulcheri, G. Flamant, I.Y. Smurov, TEMPERATURE DISTRIBUTION IN A PLASMA PILOT REACTOR FOR CARBON BLACK PRODUCTION, 15(4) (2011) 283-291.

[81] W. Cho, Y.C. Kim, S.-S. Kim, Conversion of natural gas to C2 product, hydrogen and carbon black using a catalytic plasma reaction, Journal of Industrial and Engineering Chemistry 16(1) (2010) 20-26.

[82] K.-S. Yun, B.-R. Kim, W.-S. Kang, S.-C. Jung, S.-T. Myung, S.-J. Kim, Preparation of Carbon Blacks by Liquid Phase Plasma (LPP) Process, Journal of Nanoscience and Nanotechnology 13(11) (2013) 7381-7385.

[83] N.M. Thai, S.J. Kim, Nano Carbon Black Powder Synthesized via Liquid Phase Plasma Process as a Supercapacitor Active Material, Journal of the Electrochemical Society 162(8) (2015) A1445-A1450.

[84] J.J. Yuan, R.Y. Hong, Y.Q. Wang, W.G. Feng, Low-temperature plasma preparation and application of carbon black nanoparticles, Chemical Engineering Journal 253 (2014) 107120.

[85] D.L. Sun, F. Wang, R.Y. Hong, C.R. Xie, Preparation of carbon black via arc discharge plasma enhanced by thermal pyrolysis, Diamond and Related Materials 61 (2016) 21-31. [86] X. Jiang, L. Zhang, F. Wang, Y. Liu, Q. Guo, C. Wang, Investigation of Carbon Black Production from Coal Tar via Chemical Looping Pyrolysis, Energy \& Fuels 30(4) (2016) 3535-3540.

[87] P. Toth, T. Vikström, R. Molinder, H. Wiinikka, Structure of carbon black continuously produced from biomass pyrolysis oil, Green Chemistry (2018).

[88] S. Rodat, S. Abanades, J.-L. Sans, G. Flamant, A pilot-scale solar reactor for the production of hydrogen and carbon black from methane splitting, International Journal of Hydrogen Energy 35(15) (2010) 7748-7758.

[89] S. Rodat, S. Abanades, G. Flamant, Co-production of hydrogen and carbon black from solar thermal methane splitting in a tubular reactor prototype, Solar Energy 85(4) (2011) 645652.

[90] C.-C. Liu, A.B. Walters, M.A. Vannice, Measurement of electrical properties of a carbon black, Carbon 33(12) (1995) 1699-1708.

[91] J. Sánchez-González, A. Macías-García, M.F. Alexandre-Franco, V. Gómez-Serrano, Electrical conductivity of carbon blacks under compression, Carbon 43(4) (2005) 741-747.

[92] B. Marinho, M. Ghislandi, E. Tkalya, C.E. Koning, G. de With, Electrical conductivity of compacts of graphene, multi-wall carbon nanotubes, carbon black, and graphite powder, Powder Technology 221 (2012) 351-358.

[93] I. Balberg, A comprehensive picture of the electrical phenomena in carbon blackpolymer composites, Carbon 40(2) (2002) 139-143.

[94] G.C. Psarras, Charge transport properties in carbon black/polymer composites, Journal of Polymer Science Part B: Polymer Physics 45(18) (2007) 2535-2545.

[95] Z. Zhao, W. Yu, X. He, X. Chen, The conduction mechanism of carbon black-filled poly(vinylidene fluoride) composite, Materials Letters 57(20) (2003) 3082-3088.

[96] S. Lu, D.D.L. Chung, Viscoelastic behavior of carbon black and its relationship with the aggregate size, Carbon 60 (2013) 346-355.

[97] R.N. Rothon, Particulate Fillers for Polymers, Rapra Technology2002.

[98] E.B. Sebok, R.L. Taylor, Carbon Blacks, in: K.H.J. Buschow, R.W. Cahn, M.C.

Flemings, B. Ilschner, E.J. Kramer, S. Mahajan, P. Veyssière (Eds.), Encyclopedia of

Materials: Science and Technology, Elsevier, Oxford, 2001, pp. 902-906. 
[99] A.I. Medalia, Elastomers, Reinforcement of, in: K.H.J. Buschow, R.W. Cahn, M.C. Flemings, B. Ilschner, E.J. Kramer, S. Mahajan, P. Veyssière (Eds.), Encyclopedia of Materials: Science and Technology, Elsevier, Oxford, 2001, pp. 2475-2480.

[100] Z. Ahmad, CHAPTER 7 - COATINGS, in: Z. Ahmad (Ed.), Principles of Corrosion Engineering and Corrosion Control, Butterworth-Heinemann, Oxford, 2006, pp. 382-437. [101] G. Wypych, 11 - PRINCIPLES OF STABILIZATION, in: G. Wypych (Ed.), PVC Degradation and Stabilization (Third Edition), ChemTec Publishing, Boston, 2015, pp. 287412.

[102] C. Lahousse, N. Cors, V. Ruaux, P. Grange, C. Cellier, Preparation of Pd on Carbon Black by Deposition-precipitation: Study of the Effect of the Support Functionalisation, in: E.M. Gaigneaux, M. Devillers, D.E. De Vos, S. Hermans, P.A. Jacobs, J.A. Martens, P. Ruiz (Eds.), Studies in Surface Science and Catalysis, Elsevier2006, pp. 601-608.

[103] R.A. Grimm, H. Yeh, Infrared Welding of Thermoplastics. Colored Pigments and Carbon Black Levels on Transmission of Infrared Radiation, in: R.M. Harris (Ed.), Coloring Technology for Plastics, William Andrew Publishing, Norwich, NY, 1999, pp. 277-282. [104] J.L. Koenig, Chapter 9 - Applications of high-resolution solid-state NMR spectroscopy to polymers, in: J.L. Koenig (Ed.), Spectroscopy of Polymers (Second Edition), Elsevier Science, New York, 1999, pp. 397-440.

[105] B. Rodgers, W. Waddell, Chapter 9 - The Science of Rubber Compounding, in: J.E. Mark, B. Erman, C.M. Roland (Eds.), The Science and Technology of Rubber (Fourth Edition), Academic Press, Boston, 2013, pp. 417-471.

[106] W. Zeng, S.G. Chen, B. Zhao, M.Z. Rong, M.Q. Zhang, 20 - Gas sensing conductive polymer nanocomposites filled with carbon black nanoparticles, in: S.C. Tjong, Y.W. Mai (Eds.), Physical Properties and Applications of Polymer Nanocomposites, Woodhead Publishing2010, pp. 690-722.

[107] M. Singh, R.L. Vander Wal, Nanostructure Quantification of Carbon Blacks, C 5(1) (2018) 2.

[108] D. Tasis, N. Tagmatarchis, A. Bianco, M. Prato, Chemistry of Carbon Nanotubes, Chemical Reviews 106(3) (2006) 1105-1136.

[109] X. Wang, G. Shi, An introduction to the chemistry of graphene, Physical Chemistry Chemical Physics 17(43) (2015) 28484-28504.

[110] J. Greenwood, T.H. Phan, Y. Fujita, Z. Li, O. Ivasenko, W. Vanderlinden, H. Van Gorp, W. Frederickx, G. Lu, K. Tahara, Y. Tobe, H. Uji-i, S.F.L. Mertens, S. De Feyter, Covalent Modification of Graphene and Graphite Using Diazonium Chemistry: Tunable Grafting and Nanomanipulation, ACS Nano 9(5) (2015) 5520-5535.

[111] W. Rüdorff, E. Stumpp, W. Spriessler, F.W. Siecke, Reactions of Graphite with Metal Chlorides, Angewandte Chemie International Edition in English 2(2) (1963) 67-73.

[112] M. Khaleghi Abbasabadi, A. Rashidi, S. Khodabakhshi, Benzenesulfonic acid-grafted graphene as a new and green nanoadsorbent in hydrogen sulfide removal, Journal of Natural Gas Science and Engineering 28 (2016) 87-94.

[113] P.E. Fanning, M.A. Vannice, A DRIFTS study of the formation of surface groups on carbon by oxidation, Carbon 31(5) (1993) 721-730.

[114] H. Muckenhuber, H. Grothe, The heterogeneous reaction between soot and NO2 at elevated temperature, Carbon 44(3) (2006) 546-559.

[115] A.Y. Watson, P.A. Valberg, Carbon black and soot: two different substances, AIHAJAmerican Industrial Hygiene Association 62(2) (2001) 218-228.

[116] W.R. Smith, M.H. Polley, The Oxidation of Graphitized Carbon Black, The Journal of Physical Chemistry 60(5) (1956) 689-691. 
[117] X. Chen, M. Farber, Y. Gao, I. Kulaots, E.M. Suuberg, R.H. Hurt, Mechanisms of surfactant adsorption on non-polar, air-oxidized and ozone-treated carbon surfaces, Carbon 41(8) (2003) 1489-1500.

[118] T. Kim, C. Ham, C.K. Rhee, S.-H. Yoon, M. Tsuji, I. Mochida, Effects of oxidation and heat treatment of acetylene blacks on their electrochemical double layer capacitances, Carbon 47(1) (2009) 226-233.

[119] J.-B. Donnet, P. Ehrburger, A. Voet, Etude du mecanisme d'oxydation des noirs de carbone par l'ozone en milieu aqueux, Carbon 10(6) (1972) 737-746.

[120] K. Kamegawa, K. Nishikubo, H. Yoshida, Oxidative degradation of carbon blacks with nitric acid (I) - Changes in pore and crystallographic structures, Carbon 36(4) (1998) 433441.

[121] R.C. Sosa, R.F. Parton, P.E. Neys, O. Lardinois, P.A. Jacobs, P.G. Rouxhet, Surface modification of carbon black by oxidation and its influence on the activity of immobilized catalase and iron-phthalocyanines, Journal of Molecular Catalysis A: Chemical 110(2) (1996) 141-151.

[122] G.I. Razdyakonova, O.A. Kokhanovskaya, V.A. Likholobov, Influence of Environmental Conditions on Carbon Black Oxidation by Reactive Oxygen Intermediates, Procedia Engineering 113 (2015) 43-50.

[123] R.C. Sosa, D. Masy, P.G. Rouxhet, Influence of surface properties of carbon black on the activity of adsorbed catalase, Carbon 32(7) (1994) 1369-1375.

[124] M.C. Soares, M.M. Viana, Z.L. Schaefer, V.S. Gangoli, Y. Cheng, V. Caliman, M.S. Wong, G.G. Silva, Surface modification of carbon black nanoparticles by dodecylamine: thermal stability and phase transfer in brine medium, Carbon 72 (2014) 287-295.

[125] N. Li, X. Ma, Q. Zha, K. Kim, Y. Chen, C. Song, Maximizing the number of oxygencontaining functional groups on activated carbon by using ammonium persulfate and improving the temperature-programmed desorption characterization of carbon surface chemistry, Carbon 49(15) (2011) 5002-5013.

[126] K. Kamegawa, K. Nishikubo, M. Kodama, Y. Adachi, H. Yoshida, Oxidative degradation of carbon blacks with nitric acid: II. Formation of water-soluble polynuclear aromatic compounds, Carbon 40(9) (2002) 1447-1455.

[127] A.-L. Cui, G.-X. Feng, Y.-F. Zhao, H.-Z. Kou, H. Li, G.-H. Zhu, H.-S. Hwang, H.-C. Oh, Y.-J. Kwon, D.-C. Lee, Synthesis and separation of mellitic acid and graphite oxide colloid through electrochemical oxidation of graphite in deionized water, Electrochemistry Communications 11(2) (2009) 409-412.

[128] M. Alfè, V. Gargiulo, R. Di Capua, F. Chiarella, J.-N. Rouzaud, A. Vergara, A. Ciajolo, Wet Chemical Method for Making Graphene-like Films from Carbon Black, ACS Applied Materials \& Interfaces 4(9) (2012) 4491-4498.

[129] J.C. Curtis, R.L. Taylor, G.A. Joyce, Hydrogen peroxide oxidation of carbon black, Google Patents, 2000.

[130] W. Choi, M.-T. Leu, Nitric acid uptake and decomposition on black carbon (soot) surfaces: Its implications for the upper troposphere and lower stratosphere, The Journal of Physical Chemistry A 102(39) (1998) 7618-7630.

[131] Ş.S. Bayazit, İ. İnci, Adsorption of Cu (II) ions from water by carbon nanotubes oxidized with UV-light and ultrasonication, Journal of Molecular Liquids 199 (2014) 559564.

[132] G.G. Wildgoose, P. Abiman, R.G. Compton, Characterising chemical functionality on carbon surfaces, Journal of Materials Chemistry 19(28) (2009) 4875-4886.

[133] N.P. Subramanian, X. Li, V. Nallathambi, S.P. Kumaraguru, H. Colon-Mercado, G. Wu, J.-W. Lee, B.N. Popov, Nitrogen-modified carbon-based catalysts for oxygen reduction 
reaction in polymer electrolyte membrane fuel cells, Journal of Power Sources 188(1) (2009) 38-44.

[134] W.S. Hummers Jr, R.E. Offeman, Preparation of graphitic oxide, Journal of the american chemical society 80(6) (1958) 1339-1339.

[135] S. Stankovich, D.A. Dikin, R.D. Piner, K.A. Kohlhaas, A. Kleinhammes, Y. Jia, Y. Wu, S.T. Nguyen, R.S. Ruoff, Synthesis of graphene-based nanosheets via chemical reduction of exfoliated graphite oxide, Carbon 45(7) (2007) 1558-1565.

[136] D.R. Dreyer, S. Park, C.W. Bielawski, R.S. Ruoff, The chemistry of graphene oxide, Chem. Soc. Rev. 39(1) (2010) 228-240.

[137] H. He, J. Klinowski, M. Forster, A. Lerf, A new structural model for graphite oxide, Chem. Phys. Lett. 287(1) (1998) 53-56.

[138] B.H. Suryanto, C. Zhao, Surface-oxidized carbon black as a catalyst for the water oxidation and alcohol oxidation reactions, Chemical Communications 52(38) (2016) 64396442.

[139] M.R. Acocella, M. Maggio, C. Ambrosio, N. Aprea, G. Guerra, Oxidized Carbon Black as an Activator of Transesterification Reactions under Solvent-Free Conditions, ACS Omega 2(11) (2017) 7862-7867.

[140] J. Tang, J. Yang, X. Zhou, J. Xie, G. Chen, Oxidation of acetylene black by nitric acid in hermetically sealed condition, Microporous and Mesoporous Materials 193 (2014) 54-60. [141] Y. Han, G. Hwang, S. Park, A. Gomez-Flores, E. Jo, I.-C. Eom, M. Tong, H.-J. Kim, H. Kim, Stability of carboxyl-functionalized carbon black nanoparticles: the role of solution chemistry and humic acid, Environmental Science: Nano 4(4) (2017) 800-810.

[142] K. Amornwachirabodee, N. Tantimekin, P. Pan-In, T. Palaga, P. Pienpinijtham, C.

Pipattanaboon, T. Sukmanee, P. Ritprajak, P. Charoenpat, P. Pitaksajjakul, P. Ramasoota, S. Wanichwecharungruang, Oxidized Carbon Black: Preparation, Characterization and Application in Antibody Delivery across Cell Membrane, Scientific Reports 8(1) (2018) 2489.

[143] S. Kang, A.R. Jones, J.S. Moore, S.R. White, N.R. Sottos, Microencapsulated carbon black suspensions for restoration of electrical conductivity, Advanced Functional Materials 24(20) (2014) 2947-2956.

[144] P. Xue, Y. Bao, Q. Li, C. Wu, Impact of modification of carbon black on morphology and performance of polyimide/carbon black hybrid composites, Physical Chemistry Chemical Physics 12(37) (2010) 11342-11350.

[145] N. Tsubokawa, M. Hosoya, J. Kurumada, Grafting reaction of surface carboxyl groups on carbon black with polymers having terminal hydroxyl or amino groups using N, N'dicyclohexylcarbodiimide as a condensing agent, Reactive and Functional Polymers 27(1) (1995) 75-81.

[146] N. Tsubokawa, S. Yoshikawa, K. Maruyama, T. Ogasawara, K. Saitoh, Responsibility of electric resistance of polyethyleneimine-grafted carbon black against alcohol vapor and humidity, Polymer bulletin 39(2) (1997) 217-224.

[147] J. Panchompoo, L. Aldous, R.G. Compton, Size-effects in the chemical modification of carbon black nanoparticles with 4-nitroaniline, New Journal of Chemistry 34(11) (2010) 2643-2653.

[148] S.S. Rich, J.J. Burk, C.S. Kong, C.D. Cooper, D.E. Morse, S.K. Buratto, Nitrogen functionalized carbon black: A support for Pt nanoparticle catalysts with narrow size dispersion and high surface area, Carbon 81 (2015) 115-123.

[149] H. Han, J. Lee, D.W. Park, S.E. Shim, Surface modification of carbon black by oleic acid for miniemulsion polymerization of styrene, Macromolecular research 18(5) (2010) 435441. 
[150] J.Y. Kim, J.-Y. Oh, K.-S. Suh, Voltage switchable surface-modified carbon black nanoparticles for dual-particle electrophoretic displays, Carbon 66 (2014) 361-368.

[151] M. Toupin, D. Bélanger, Spontaneous functionalization of carbon black by reaction with 4-nitrophenyldiazonium cations, Langmuir 24(5) (2008) 1910-1917.

[152] S.A. Goetz, D.T. Nguyen, A.P. Esser-Kahn, Surface modification of carbon black nanoparticles enhances photothermal separation and release of CO 2, Carbon 105 (2016) 126135.

[153] C.1. Shepherd, E. Hadzifejzovic, F. Shkal, K. Jurkschat, J. Moghal, E.M. Parker, M. Sawangphruk, D.R. Slocombe, J.S. Foord, M.G. Moloney, New routes to functionalize carbon black for polypropylene nanocomposites, Langmuir 32(31) (2016) 7917-7928.

[154] K. Bergemann, E. Fanghanel, B. Knackfuß, T. Lüthge, G. Schukat, Modification of carbon black properties by reaction with maleic acid derivatives, Carbon 11(42) (2004) 23382340.

[155] M. Levy, M. Szwarc, Methyl affinities of aromatic compounds, The Journal of Chemical Physics 22(9) (1954) 1621-1622.

[156] Q. Li, G. Wu, Y. Ma, C. Wu, Grafting modification of carbon black by trapping macroradicals formed by sonochemical degradation, Carbon 45(12) (2007) 2411-2416. [157] M. Sansotera, C.L. Bianchi, G. Lecardi, G. Marchionni, P. Metrangolo, G. Resnati, W. Navarrini, Highly hydrophobic carbon black obtained by covalent linkage of perfluorocarbon and perfluoropolyether chains on the carbon surface, Chemistry of Materials 21(19) (2009) 4498-4504.

[158] N. Tsubokawa, Functionalization of carbon black by surface grafting of polymers, Progress in Polymer science 17(3) (1992) 417-470.

[159] S. Yoshikawa, S. Machida, N. Tsubokawa, Polymer grafting onto carbon black by use of TEMPO- terminated polystyrene with controlled molecular weight, Journal of Polymer Science Part A: Polymer Chemistry 36(17) (1998) 3165-3172.

[160] J.-S. Wang, K. Matyjaszewski, Controlled/" living" radical polymerization. Atom transfer radical polymerization in the presence of transition-metal complexes, Journal of the American Chemical Society 117(20) (1995) 5614-5615.

[161] M. Kato, M. Kamigaito, M. Sawamoto, T. Higashimura, Polymerization of methyl methacrylate with the carbon tetrachloride/dichlorotris-(triphenylphosphine) ruthenium (II)/methylaluminum bis (2, 6-di-tert-butylphenoxide) initiating system: possibility of living radical polymerization, Macromolecules 28(5) (1995) 1721-1723.

[162] K. Ohno, K.-m. Koh, Y. Tsujii, T. Fukuda, Synthesis of gold nanoparticles coated with well-defined, high-density polymer brushes by surface-initiated living radical polymerization, Macromolecules 35(24) (2002) 8989-8993.

[163] T. Liu, S. Jia, T. Kowalewski, K. Matyjaszewski, R. Casado-Portilla, J. Belmont, Grafting poly (n-butyl acrylate) from a functionalized carbon black surface by atom transfer radical polymerization, Langmuir 19(16) (2003) 6342-6345.

[164] T. Liu, R. Casado- Portilla, J. Belmont, K. Matyjaszewski, ATRP of butyl acrylates from functionalized carbon black surfaces, Journal of Polymer Science Part A: Polymer Chemistry 43(20) (2005) 4695-4709.

[165] T. Liu, S. Jia, T. Kowalewski, K. Matyjaszewski, R. Casado-Portilla, J. Belmont, Water-dispersible carbon black nanocomposites prepared by surface-initiated atom transfer radical polymerization in protic media, Macromolecules 39(2) (2006) 548-556.

[166] H. He, M. Zhong, D. Konkolewicz, K. Yacatto, T. Rappold, G. Sugar, N.E. David, K. Matyjaszewski, Carbon black functionalized with hyperbranched polymers: synthesis, characterization, and application in reversible CO2 capture, Journal of Materials Chemistry A 1(23) (2013) 6810-6821. 
[167] Q. Yang, L. Wang, W. Xiang, J. Zhou, L. Deng, J. Li, Preparation of core-shell carbon black nanoparticles and their crystallization-induced orientation, European polymer journal 43(5) (2007) 1718-1723.

[168] Q. Yang, L. Wang, W.d. Xiang, J.f. Zhou, Q.h. Tan, Preparation of polymer- grafted carbon black nanoparticles by surface- initiated atom transfer radical polymerization, Journal of Polymer Science Part A: Polymer Chemistry 45(15) (2007) 3451-3459.

[169] J. Cheng, L. Wang, J. Huo, H. Yu, Q. Yang, L. Deng, Study on synthesis of poly

(N- isopropylacrylamide) grafted carbon black and temperature- dependence conductivity of grafted carbon black/epoxy composites, Journal of Polymer Science Part B: Polymer Physics 46(15) (2008) 1529-1535.

[170] C.-C. Hwang, L. Wang, W. Lu, G. Ruan, G.C. Kini, C. Xiang, E.L. Samuel, W. Shi, A.T. Kan, M.S. Wong, Highly stable carbon nanoparticles designed for downhole hydrocarbon detection, Energy \& Environmental Science 5(8) (2012) 8304-8309.

[171] S. Hayashi, S. Handa, Y. Oshibe, T. Yamamoto, N. Tsubokawa, Grafting of polymers onto carbon black surface by the trapping of polymer radicals formed by the decomposition of peroxide polymers, Polymer journal 27(6) (1995) 623.

[172] S. Hayashi, S. Handa, N. Tsubokawa, Introduction of peroxide groups onto carbon black surface by radical trapping and radical graft polymerization of vinyl monomers initiated by the surface peroxide groups, Journal of Polymer Science Part A: Polymer Chemistry 34(8) (1996) 1589-1595.

[173] S. Hayashi, A. Naitoh, S. Machida, M. Okazaki, K. Maruyama, N. Tsubokawa, Grafting of polymers onto a carbon black surface by the trapping of polymer radicals, Applied organometallic chemistry 12(10- 11) (1998) 743-748.

[174] H. Iwata, T. Nakanoya, H. Morohashi, J. Chen, T. Yamauchi, N. Tsubokawa, Novel gas and contamination sensor materials from polyamide-block-poly (ethylene oxide)-grafted carbon black, Sensors and Actuators B: Chemical 113(2) (2006) 875-882.

[175] H. Liu, S. Wang, Y. Xiao, X. Li, Studies on the dispersity of polymethacrylate-grafted carbon black in a non-aqueous medium: the influence of monomer structure, Journal of Materials Science: Materials in Electronics 27(2) (2016) 2022-2030.

[176] K. Kinoshita, J. Bett, Electrochemical oxidation of carbon black in concentrated phosphoric acid at 135 C, Carbon 11(3) (1973) 237-247.

[177] A.M. Chaparro, N. Mueller, C. Atienza, L. Daza, Study of electrochemical instabilities of PEMFC electrodes in aqueous solution by means of membrane inlet mass spectrometry, $\mathrm{J}$. Electroanal. Chem. 591(1) (2006) 69-73.

[178] A. Cuesta, A. Martínez-Alonso, J. Tascon, Carbon reactivity in an oxygen plasma: a comparison with reactivity in molecular oxygen, Carbon 39(8) (2001) 1135-1146.

[179] S.-J. Park, K.-S. Cho, S.-K. Ryu, Filler-elastomer interactions: influence of oxygen plasma treatment on surface and mechanical properties of carbon black/rubber composites, Carbon 41(7) (2003) 1437-1442.

[180] B. Zhang, Y. Chen, F. Wang, R. Hong, Surface modification of carbon black for the reinforcement of polycarbonate/acrylonitrile-butadiene-styrene blends, Applied Surface Science 351 (2015) 280-288.

[181] M. Nagornaya, G. Razdyakonova, S.Y. Khodakova, The effect of functional groups of carbon black on rubber properties, Procedia Engineering 152 (2016) 563-569.

[182] J.L. Figueiredo, M.F.R. Pereira, M.M.A. Freitas, J.J.M. Órfão, Modification of the surface chemistry of activated carbons, Carbon 37(9) (1999) 1379-1389.

[183] J.M. Peña, N.S. Allen, M. Edge, C.M. Liauw, S.R. Hoon, B. Valange, R.I. Cherry, Analysis of radical content on carbon black pigments by electron spin resonance: influence of 
functionality, thermal treatment and adsorption of acidic and basic probes, Polymer Degradation and Stability 71(1) (2000) 153-170.

[184] D. Leistenschneider, K. Zürbes, C. Schneidermann, S. Grätz, S. Oswald, K. Wegner, B. Klemmed, L. Giebeler, A. Eychmüller, L. Borchardt, Mechanochemical Functionalization of Carbon Black at Room Temperature, C 4(1) (2018) 14.

[185] A. Kirubakaran, S. Jain, R.K. Nema, A review on fuel cell technologies and power electronic interface, Renewable and Sustainable Energy Reviews 13(9) (2009) 2430-2440. [186] K.A. Mauritz, R.B. Moore, State of Understanding of Nafion, Chemical Reviews 104(10) (2004) 4535-4586.

[187] A.L. Dicks, The role of carbon in fuel cells, Journal of Power Sources 156(2) (2006) 128-141.

[188] P. Trogadas, T.F. Fuller, P. Strasser, Carbon as catalyst and support for electrochemical energy conversion, Carbon 75 (2014) 5-42.

[189] E. Antolini, Carbon supports for low-temperature fuel cell catalysts, Applied Catalysis B: Environmental 88(1) (2009) 1-24.

[190] E. Auer, A. Freund, J. Pietsch, T. Tacke, Carbons as supports for industrial precious metal catalysts, Applied Catalysis A: General 173(2) (1998) 259-271.

[191] M. Wissler, Graphite and carbon powders for electrochemical applications, Journal of Power Sources 156(2) (2006) 142-150.

[192] S. Sharma, B.G. Pollet, Support materials for PEMFC and DMFC electrocatalysts-A review, Journal of Power Sources 208 (2012) 96-119.

[193] M. Uchida, Y. Aoyama, M. Tanabe, N. Yanagihara, N. Eda, A. Ohta, Influences of both carbon supports and heat- treatment of supported catalyst on electrochemical oxidation of methanol, Journal of the Electrochemical Society 142(8) (1995) 2572-2576.

[194] D.H. Kwak, Y.W. Lee, K.H. Lee, A.R. Park, J.S. Moon, K.W. Park, One-Step

Synthesis of Hexapod Pt Nanoparticles Deposited on Carbon Black for Improved Methanol Electrooxidation, International Journal of Electrochemical Science 8(4) (2013) 5102-5107.

[195] M. Carmo, M. Linardi, J.G. Rocha Poco, H2O2 treated carbon black as electrocatalyst support for polymer electrolyte membrane fuel cell applications, International Journal of Hydrogen Energy 33(21) (2008) 6289-6297.

[196] A. Valera-Medina, H. Xiao, M. Owen-Jones, W.I.F. David, P.J. Bowen, Ammonia for power, Progress in Energy and Combustion Science 69 (2018) 63-102.

[197] A. Serov, C. Kwak, Direct hydrazine fuel cells: A review, Applied Catalysis B:

Environmental 98(1) (2010) 1-9.

[198] K. Horita, Y. Nishibori, T. Ohshima, Surface modification of carbon black by anodic oxidation and electrochemical characterization, Carbon 34(2) (1996) 217-222.

[199] M. Bron, Carbon black supported gold nanoparticles for oxygen electroreduction in acidic electrolyte solution, Journal of Electroanalytical Chemistry 624(1) (2008) 64-68. [200] M. Ciobanu, A.-M. Lepadatu, S. Asaftei, Chemical and Electrochemical Studies of Carbon Black Surface by Treatment with Ozone and Nitrogen Oxide, Materials Today: Proceedings 3 (2016) S252-S257.

[201] C.K. Poh, S.H. Lim, H. Pan, J. Lin, J.Y. Lee, Citric acid functionalized carbon materials for fuel cell applications, Journal of Power Sources 176(1) (2008) 70-75. [202] K. Guerin, M. Dubois, A. Houdayer, A. Hamwi, Applicative performances of fluorinated carbons through fluorination routes: A review, Journal of Fluorine Chemistry 134 (2012) 11-17.

[203] Y.-S. Lee, Syntheses and properties of fluorinated carbon materials, Journal of Fluorine Chemistry 128(4) (2007) 392-403. 
[204] X.L. Wang, H.M. Zhang, J.L. Zhang, H.F. Xu, Z.Q. Tian, J. Chen, H.X. Zhong, Y.M. Liang, B.L. Yi, Micro-porous layer with composite carbon black for PEM fuel cells, Electrochimica Acta 51(23) (2006) 4909-4915.

[205] G. Kornienko, N. Chaenko, I. Vasil'eva, G. Kolyagin, V. Kornienko, Effect of the thickness and porosity of carbon-black gas-diffusion air electrodes on the efficiency of hydrogen peroxide synthesis from atmospheric oxygen, Russian Journal of Applied Chemistry 83(2) (2010) 253-257.

[206] Y. Li, Y. Li, E. Zhu, T. McLouth, C.-Y. Chiu, X. Huang, Y. Huang, Stabilization of High-Performance Oxygen Reduction Reaction Pt Electrocatalyst Supported on Reduced Graphene Oxide/Carbon Black Composite, Journal of the American Chemical Society 134(30) (2012) 12326-12329.

[207] S.H. Park, H.N. Yang, D.C. Lee, K.W. Park, W.J. Kim, Electrochemical properties of polyethyleneimine-functionalized Pt-PEI/carbon black as a catalyst for polymer electrolyte membrane fuel cell, Electrochimica Acta 125 (2014) 141-148.

[208] J. Park, S. Kim, Electrochemical Analysis of Polyethyleneimine-Conductive Carbon Black Supports for Pt-Pd Electrocatalysts, Journal of nanoscience and nanotechnology 15(2) (2015) 1610-1613.

[209] N. He, C. Qin, R. Wang, S. Ma, Y. Wang, T. Qi, Electro-catalysis of carbon black or titanium sub-oxide supported Pd-Gd towards formic acid electro-oxidation, RSC Advances 6(73) (2016) 68989-68996.

[210] J.-Y. Wang, Y.-Y. Kang, H. Yang, W.-B. Cai, Boron-Doped Palladium Nanoparticles on Carbon Black as a Superior Catalyst for Formic Acid Electro-oxidation, The Journal of Physical Chemistry C 113(19) (2009) 8366-8372.

[211] J.-i. Ozaki, T. Anahara, N. Kimura, C. Ida, A. Oya, B.B. Bokhonov, M.A. Korchagin, M. Sakashita, Preparation of BN-doped carbon blacks by mechanochemical alloying of carbon and h-BN and its use as a catalyst for the oxygen reduction, TANSO 2007(228) (2007) 153-157.

[212] F. Jaouen, J.-P. Dodelet, Non-Noble Electrocatalysts for O2 Reduction: How Does Heat Treatment Affect Their Activity and Structure? Part I. Model for Carbon Black Gasification by NH3: Parametric Calibration and Electrochemical Validation, The Journal of Physical Chemistry C 111(16) (2007) 5963-5970.

[213] J. Herranz, M. Lefèvre, N. Larouche, B. Stansfield, J.-P. Dodelet, Step-by-Step Synthesis of Non-Noble Metal Electrocatalysts for O2 Reduction under Proton Exchange Membrane Fuel Cell Conditions, The Journal of Physical Chemistry C 111(51) (2007) 19033-19042.

[214] Y. Si, M.G. Park, Z.P. Cano, Z. Xiong, Z. Chen, Heavily nitrogen-doped acetylene black as a high-performance catalyst for oxygen reduction reaction, Carbon 117 (2017) 1219.

[215] X. Su, Y. Yao, J. Tian, J. Liu, Z. Wang, Y. You, L. Huang, C. Wu, Investigation of the durability of a poly-p-phenylenediamine/carbon black composite for the oxygen reduction reaction, Chinese Journal of Catalysis 37(7) (2016) 1096-1102.

[216] X. Zhang, Y. Chen, J. Wang, Q. Zhong, Nitrogen and fluorine dual- doped carbon black as an efficient cathode catalyst for oxygen reduction reaction in neutral medium, ChemistrySelect 1(4) (2016) 696-702.

[217] K. Loganathan, D. Bose, D. Weinkauf, Surface modification of carbon black by nitrogen and allylamine plasma treatment for fuel cell electrocatalyst, International Journal of Hydrogen Energy 39(28) (2014) 15766-15771. 
[218] H. Qian, H. Huang, X. Wang, Design and synthesis of palladium/graphitic carbon nitride/carbon black hybrids as high-performance catalysts for formic acid and methanol electrooxidation, Journal of Power Sources 275 (2015) 734-741.

[219] H. Dong, X. Zhang, H. Yu, H. Yu, Graphene-doped carbon black gas diffusion electrode for nonmetallic electrochemical advanced oxidation process under mild conditions, Environmental Technology (2017) 1-8.

[220] T.B. Reddy, Linden's handbook of batteries, McGraw-hill New York2011.

[221] Y. Idemoto, T. Takahashi, N. Ishida, M. Nakayama, N. Kitamura, Synthesis, Crystal Structure Analysis, and Electrochemical Properties of Rock-Salt Type MgxNiyCozO2 as a Cathode Material for Mg Rechargeable Batteries, Inorganic Chemistry 58(9) (2019) 56645670.

[222] A.P. Vijaya Kumar Saroja, S.S. Samantaray, R. Sundara, A room temperature multivalent rechargeable iron ion battery with an ether based electrolyte: a new type of postlithium ion battery, Chemical Communications 55(70) (2019) 10416-10419.

[223] J. Fu, R. Liang, G. Liu, A. Yu, Z. Bai, L. Yang, Z. Chen, Recent Progress in Electrically Rechargeable Zinc-Air Batteries, Advanced Materials 31(31) (2019) 1805230. [224] D. Coffin, J. Horowitz, The Supply Chain for Electric Vehicle Batteries, J. Int'l Com. \& Econ. (2018) 1.

[225] V. Etacheri, R. Marom, R. Elazari, G. Salitra, D. Aurbach, Challenges in the development of advanced Li-ion batteries: a review, Energy \& Environmental Science 4(9) (2011) 3243-3262.

[226] X.K. Huang, H.J. Yue, A. Attia, Y. Yang, Preparation and properties of manganese oxide/carbon composites by reduction of potassium permanganate with acetylene black, Journal of the Electrochemical Society 154(1) (2007) A26-A33.

[227] R. Dominko, M. Gaberscek, J. Drofenik, M. Bele, S. Pejovnik, J. Jamnik, The role of carbon black distribution in cathodes for Li ion batteries, Journal of Power Sources 119-121 (2003) 770-773.

[228] L. Zhang, M. Zhang, Y. Wang, Z. Zhang, G. Kan, C. Wang, Z. Zhong, F. Su, Graphitized porous carbon microspheres assembled with carbon black nanoparticles as improved anode materials in Li-ion batteries, Journal of Materials Chemistry A 2(26) (2014) 10161-10168.

[229] S. Lu, H. Wang, J. Zhou, X. Wu, W. Qin, Atomic layer deposition of ZnO on carbon black as nanostructured anode materials for high-performance lithium-ion batteries, Nanoscale 9(3) (2017) 1184-1192.

[230] K. Hanvin, Y. Yongsub, L. Young-Chan, L. Myeong-Hoon, S. Nagahiro, K. Jun, Synthesis of silicon-carbon black composite as anode material for lithium ion battery, Japanese Journal of Applied Physics 57(1) (2018) 0102B2.

[231] X. Li, X. Li, M.N. Banis, B. Wang, A. Lushington, X. Cui, R. Li, T.-K. Sham, X. Sun, Tailoring interactions of carbon and sulfur in Li-S battery cathodes: significant effects of carbon-heteroatom bonds, Journal of Materials Chemistry A 2(32) (2014) 12866-12872.

[232] G. Jin, Z. Mingang, Y. Shijian, Y. Xiaoyan, W. Shiwei, Electrochemical properties of modified acetylene black/sulfur composite cathode material for lithium/sulfur batteries, Ionics (2017).

[233] W. Qin, S. Lu, Z. Wang, X. Wu, Edge-functionalized acetylene black anchoring sulfur for high-performance Li-S batteries, Journal of Energy Chemistry 26(3) (2017) 448-453. [234] M.J. Lacey, F. Jeschull, K. Edström, D. Brandell, Porosity Blocking in Highly Porous Carbon Black by PVdF Binder and Its Implications for the Li-S System, The Journal of Physical Chemistry C 118(45) (2014) 25890-25898. 
[235] X.-B. Yang, W. Zhu, K. Qin, H.-Y. Wang, Preparation of lamellar carbon matrix for sulfur as cathode material of lithium-sulfur batteries, Electrochimica Acta 143 (2014) 374382.

[236] L. Fransson, T. Eriksson, K. Edström, T. Gustafsson, J.O. Thomas, Influence of carbon black and binder on Li-ion batteries, Journal of Power Sources 101(1) (2001) 1-9.

[237] X. Yang, W. Zhu, G. Cao, X. Zhao, Preparation of reduced carbon-wrapped carbonsulfur composite as cathode material of lithium-sulfur batteries, RSC Advances 5(114) (2015) 93926-93936.

[238] X. Ji, L.F. Nazar, Advances in Li-S batteries, Journal of Materials Chemistry 20(44) (2010) 9821-9826.

[239] C.K. Chan, H. Peng, G. Liu, K. McIlwrath, X.F. Zhang, R.A. Huggins, Y. Cui, Highperformance lithium battery anodes using silicon nanowires, Nature Nanotechnology 3 (2007) 31.

[240] L.-F. Cui, Y. Yang, C.-M. Hsu, Y. Cui, Carbon-Silicon Core-Shell Nanowires as High Capacity Electrode for Lithium Ion Batteries, Nano Letters 9(9) (2009) 3370-3374.

[241] J. Kong, W.A. Yee, Y. Wei, L. Yang, J.M. Ang, S.L. Phua, S.Y. Wong, R. Zhou, Y. Dong, X. Li, X. Lu, Silicon nanoparticles encapsulated in hollow graphitized carbon nanofibers for lithium ion battery anodes, Nanoscale 5(7) (2013) 2967-2973.

[242] H. Li, X. Huang, L. Chen, Z. Wu, Y. Liang, A high capacity nano Si composite anode material for lithium rechargeable batteries, Electrochemical and Solid-State Letters 2(11) (1999) 547-549.

[243] G.-L. Xu, Y. Li, T. Ma, Y. Ren, H.-H. Wang, L. Wang, J. Wen, D. Miller, K. Amine, $\mathrm{Z}$. Chen, PEDOT-PSS coated $\mathrm{ZnO} / \mathrm{C}$ hierarchical porous nanorods as ultralong-life anode material for lithium ion batteries, Nano Energy 18 (2015) 253-264.

[244] S.E. Kesler, P.W. Gruber, P.A. Medina, G.A. Keoleian, M.P. Everson, T.J. Wallington, Global lithium resources: Relative importance of pegmatite, brine and other deposits, Ore Geology Reviews 48 (2012) 55-69.

[245] J. Górka, C. Vix-Guterl, C. Matei Ghimbeu, Recent Progress in Design of BiomassDerived Hard Carbons for Sodium Ion Batteries, C 2(4) (2016) 24.

[246] R. Alcántara, J.M. Jiménez-Mateos, P. Lavela, J.L. Tirado, Carbon black: a promising electrode material for sodium-ion batteries, Electrochemistry Communications 3(11) (2001) 639-642.

[247] M. Kruk, M. Jaroniec, Gas adsorption characterization of ordered organic-inorganic nanocomposite materials, Chemistry of Materials 13(10) (2001) 3169-3183.

[248] Y. Zhai, Y. Dou, D. Zhao, P.F. Fulvio, R.T. Mayes, S. Dai, Carbon materials for chemical capacitive energy storage, Advanced materials 23(42) (2011) 4828-4850.

[249] P. Simon, Y. Gogotsi, Materials for electrochemical capacitors, Nature Materials 7(11) (2008) 845-854.

[250] Y. Zhang, H. Feng, X. Wu, L. Wang, A. Zhang, T. Xia, H. Dong, X. Li, L. Zhang, Progress of electrochemical capacitor electrode materials: A review, International Journal of Hydrogen Energy 34(11) (2009) 4889-4899.

[251] M. Zhi, C. Xiang, J. Li, M. Li, N. Wu, Nanostructured carbon-metal oxide composite electrodes for supercapacitors: a review, Nanoscale 5(1) (2013) 72-88.

[252] C. Portet, G. Yushin, Y. Gogotsi, Electrochemical performance of carbon onions, nanodiamonds, carbon black and multiwalled nanotubes in electrical double layer capacitors, Carbon 45(13) (2007) 2511-2518.

[253] Y.V. Surovikin, A.G. Shaitanov, I.V. Resanov, A.V. Syr'eva, The Properties of Nanodispersed Carbon Black Particles After Thermal Treatment, Procedia Engineering 113 (2015) 519-524. 
[254] O.O. Mykhaylyk, Y.M. Solonin, D.N. Batchelder, R. Brydson, Transformation of nanodiamond into carbon onions: A comparative study by high-resolution transmission electron microscopy, electron energy-loss spectroscopy, x-ray diffraction, small-angle x-ray scattering, and ultraviolet Raman spectroscopy, Journal of Applied Physics 97(7) (2005) 074302.

[255] P. Ganesh, P.R.C. Kent, V. Mochalin, Formation, characterization, and dynamics of onion-like carbon structures for electrical energy storage from nanodiamonds using reactive force fields, Journal of Applied Physics 110(7) (2011) 073506.

[256] J. Yan, T. Wei, B. Shao, F. Ma, Z. Fan, M. Zhang, C. Zheng, Y. Shang, W. Qian, F. Wei, Electrochemical properties of graphene nanosheet/carbon black composites as electrodes for supercapacitors, Carbon 48(6) (2010) 1731-1737.

[257] W. Ma, S. Chen, S. Yang, M. Zhu, Hierarchically porous carbon black/graphene hybrid fibers for high performance flexible supercapacitors, RSC Advances 6(55) (2016) 5011250118.

[258] P.F. Fulvio, R.T. Mayes, X. Wang, S.M. Mahurin, J.C. Bauer, V. Presser, J.

McDonough, Y. Gogotsi, S. Dai, "Brick-and-Mortar" Self-Assembly Approach to Graphitic Mesoporous Carbon Nanocomposites, Advanced Functional Materials 21(12) (2011) 22082215.

[259] J.M. Szeifert, D. Fattakhova-Rohlfing, D. Georgiadou, V. Kalousek, J. Rathouský, D. Kuang, S. Wenger, S.M. Zakeeruddin, M. Grätzel, T. Bein, "Brick and Mortar" Strategy for the Formation of Highly Crystalline Mesoporous Titania Films from Nanocrystalline Building Blocks, Chemistry of Materials 21(7) (2009) 1260-1265.

[260] C. Yang, P. Liu, T. Wang, Well-Defined Core-Shell Carbon Black/Polypyrrole Nanocomposites for Electrochemical Energy Storage, ACS Applied Materials \& Interfaces 3(4) (2011) 1109-1114.

[261] R. Ou, S. Gupta, C.A. Parker, R.A. Gerhardt, Fabrication and Electrical Conductivity of Poly(methyl methacrylate) (PMMA)/Carbon Black (CB) Composites: Comparison between an Ordered Carbon Black Nanowire-Like Segregated Structure and a Randomly Dispersed Carbon Black Nanostructure, The Journal of Physical Chemistry B 110(45) (2006) 22365-22373.

[262] V. Presser, C.R. Dennison, J. Campos, K.W. Knehr, E.C. Kumbur, Y. Gogotsi, The Electrochemical Flow Capacitor: A New Concept for Rapid Energy Storage and Recovery, Adv. Energy Mater. 2(7) (2012) 895-902.

[263] M. Boota, K. Hatzell, M. Beidaghi, C. Dennison, E. Kumbur, Y. Gogotsi, Activated carbon spheres as a flowable electrode in electrochemical flow capacitors, Journal of The Electrochemical Society 161(6) (2014) A1078-A1083.

[264] J.W. Campos, M. Beidaghi, K.B. Hatzell, C.R. Dennison, B. Musci, V. Presser, E.C. Kumbur, Y. Gogotsi, Investigation of carbon materials for use as a flowable electrode in electrochemical flow capacitors, Electrochimica Acta 98 (2013) 123-130.

[265] C. Zhang, K.B. Hatzell, M. Boota, B. Dyatkin, M. Beidaghi, D. Long, W. Qiao, E.C. Kumbur, Y. Gogotsi, Highly porous carbon spheres for electrochemical capacitors and capacitive flowable suspension electrodes, Carbon 77 (2014) 155-164.

[266] M. Boota, K. Hatzell, M. Alhabeb, E. Kumbur, Y. Gogotsi, Graphene-containing flowable electrodes for capacitive energy storage, Carbon 92 (2015) 142-149.

[267] Y.-F. Du, P.-W. Shi, Q.-Y. Li, Y.-C. Li, C.-F. Wu, Effect of poly(sodium 4styrenesulfonate) modified carbon black on the dispersion and properties of waterborne polyurethane nanocomposites, Colloids and Surfaces A: Physicochemical and Engineering Aspects 454 (2014) 1-7. 
[268] P.-W. Shi, Q.-Y. Li, Y.-C. Li, C.-F. Wu, Preparation and characterization of poly(sodium 4-styrenesulfonate)-decorated hydrophilic carbon black by one-step in situ ball milling, Colloids and Surfaces A: Physicochemical and Engineering Aspects 443 (2014) 135140.

[269] J. Yang, B. Tang, W. Qiu, S. Zhang, Controlled dispersion and precipitation of carbon black by a $\mathrm{pH}$-responsive polyampholyte containing amino groups and aryl sulfonates, Carbon 50(15) (2012) 5621-5624.

[270] K.B. Hatzell, M. Beidaghi, J.W. Campos, C.R. Dennison, E.C. Kumbur, Y. Gogotsi, A high performance pseudocapacitive suspension electrode for the electrochemical flow capacitor, Electrochimica Acta 111 (2013) 888-897.

[271] L. Li, W. Zhu, P. Zhang, Z. Chen, W. Han, Photocatalytic oxidation and ozonation of catechol over carbon-black-modified nano-TiO2 thin films supported on $\mathrm{Al}$ sheet, Water Research 37(15) (2003) 3646-3651.

[272] R. Leary, A. Westwood, Carbonaceous nanomaterials for the enhancement of TiO2 photocatalysis, Carbon 49(3) (2011) 741-772.

[273] Y. Cong, X. Li, Z. Dong, G. Yuan, Z. Cui, J. Zhang, Core-shell structured carbon black@TiO2 microsphere with enhanced visible light photocatalytic activity, Materials Letters 138 (2015) 200-203.

[274] A.L. Linsebigler, G. Lu, J.T. Yates, Photocatalysis on TiO2 Surfaces: Principles, Mechanisms, and Selected Results, Chemical Reviews 95(3) (1995) 735-758.

[275] X. Li, J. Yu, M. Jaroniec, Hierarchical photocatalysts, Chem. Soc. Rev. 45(9) (2016) 2603-2636.

[276] B. O'Regan, M. Grätzel, A low-cost, high-efficiency solar cell based on dye-sensitized colloidal TiO2 films, Nature 353(6346) (1991) 737-740.

[277] T.W. Hamann, R.A. Jensen, A.B.F. Martinson, H. Van Ryswyk, J.T. Hupp, Advancing beyond current generation dye-sensitized solar cells, Energy \& Environmental Science 1(1) (2008) 66-78.

[278] C.-C. Mao, H.-S. Weng, Promoting effect of adding carbon black to TiO2 for aqueous photocatalytic degradation of methyl orange, Chemical Engineering Journal 155(3) (2009) 744-749.

[279] M.E. Rincón, M.E. Trujillo-Camacho, A.K. Cuentas-Gallegos, Sol-gel titanium oxides sensitized by nanometric carbon blacks: Comparison with the optoelectronic and photocatalytic properties of physical mixtures, Catalysis Today 107-108 (2005) 606-611. [280] M.E. Rincón, M.E. Trujillo-Camacho, A.K. Cuentas-Gallegos, N. Casillas, Surface characterization of nanostructured $\mathrm{TiO} 2$ and carbon blacks composites by dye adsorption and photoelectrochemical studies, Applied Catalysis B: Environmental 69(1) (2006) 65-74. [281] M.E. Rincón, M.E. Trujillo, J. Ávalos, N. Casillas, Photoelectrochemical processes at interfaces of nanostructured $\mathrm{TiO} 2 /$ carbon black composites studied by scanning photoelectrochemical microscopy, Journal of Solid State Electrochemistry 11(9) (2007) 1287-1294.

[282] J. Lim, S.Y. Ryu, J. Kim, Y. Jun, A study of TiO2/carbon black composition as counter electrode materials for dye-sensitized solar cells, Nanoscale Research Letters 8(1) (2013) 227.

[283] T.N. Murakami, S. Ito, Q. Wang, M.K. Nazeeruddin, T. Bessho, I. Cesar, P. Liska, R. Humphry-Baker, P. Comte, P. Pechy, M. Gratzel, Highly efficient dye-sensitized solar cells based on carbon black counter electrodes, Journal of the Electrochemical Society 153(12) (2006) A2255-A2261. 
[284] C.-S. Wu, S. Venkatesan, T.-W. Chang, Y.-L. Lee, Platinum/carbon black composites as counter electrodes for high-performance dye-sensitized solar cells, Journal of Solid State Electrochemistry 22(1) (2018) 255-262.

[285] C.-C. Wang, S.-Y. Lu, Carbon black-derived graphene quantum dots composited with carbon aerogel as a highly efficient and stable reduction catalyst for the iodide/tri-iodide couple, Nanoscale 7(3) (2015) 1209-1215.

[286] S. Zhu, Y. Song, X. Zhao, J. Shao, J. Zhang, B. Yang, The photoluminescence mechanism in carbon dots (graphene quantum dots, carbon nanodots, and polymer dots): current state and future perspective, Nano Research 8(2) (2015) 355-381.

[287] J. Shen, Y. Zhu, X. Yang, C. Li, Graphene quantum dots: emergent nanolights for bioimaging, sensors, catalysis and photovoltaic devices, Chemical Communications 48(31) (2012) 3686-3699.

[288] Y. Zhang, J. Hao, J. Li, C. Hao, Theoretical study of triiodide reduction reaction on nitrogen-doped graphene for dye-sensitized solar cells, Theoretical Chemistry Accounts 135(1) (2016) 23.

[289] L. Tang, R. Ji, X. Li, G. Bai, C.P. Liu, J. Hao, J. Lin, H. Jiang, K.S. Teng, Z. Yang, S.P. Lau, Deep Ultraviolet to Near-Infrared Emission and Photoresponse in Layered NDoped Graphene Quantum Dots, ACS Nano 8(6) (2014) 6312-6320.

[290] Y. Li, Y. Zhao, H. Cheng, Y. Hu, G. Shi, L. Dai, L. Qu, Nitrogen-Doped Graphene Quantum Dots with Oxygen-Rich Functional Groups, Journal of the American Chemical Society 134(1) (2012) 15-18.

[291] G. Kang, J. Choi, T. Park, Pt-Free Counter Electrodes with Carbon Black and 3D Network Epoxy Polymer Composites, Scientific Reports 6 (2016) 22987.

[292] D. Han, Z. Meng, D. Wu, C. Zhang, H. Zhu, Thermal properties of carbon black aqueous nanofluids for solar absorption, Nanoscale Research Letters 6(1) (2011) 457. [293] O. Neumann, A.S. Urban, J. Day, S. Lal, P. Nordlander, N.J. Halas, Solar Vapor Generation Enabled by Nanoparticles, ACS Nano 7(1) (2013) 42-49.

[294] L.V.W. Randy, D.M. Steven, P. Vladimir, Nanocarbon nanofluids: morphology and nanostructure comparisons, Nanotechnology 20(10) (2009) 105702.

[295] S.A. Goetz, D.T. Nguyen, A.P. Esser-Kahn, Surface modification of carbon black nanoparticles enhances photothermal separation and release of CO2, Carbon 105 (2016) 126135.

[296] D.T. Nguyen, R. Truong, R. Lee, S.A. Goetz, A.P. Esser-Kahn, Photothermal release of $\mathrm{CO} 2$ from capture solutions using nanoparticles, Energy \& Environmental Science 7(8) (2014) 2603-2607.

[297] R.S. Haszeldine, Carbon Capture and Storage: How Green Can Black Be?, Science 325(5948) (2009) 1647.

[298] P.C. Hillesheim, S. Dai, S. Mahurin, P. Fulvio, Task specific ionic liquids for postcombustion $\mathrm{CO} 2$ sequestration and gas separation applications, Abstracts of Papers of the American Chemical Society 244 (2012).

[299] P.C. Hillesheim, S.M. Mahurin, P.F. Fulvio, J.S. Yeary, Y. Oyola, D.-e. Jiang, S. Dai, Synthesis and Characterization of Thiazolium-Based Room Temperature Ionic Liquids for Gas Separations, Industrial \& Engineering Chemistry Research 51(35) (2012) 11530-11537. [300] P.C. Hillesheim, J.A. Singh, S.M. Mahurin, P.F. Fulvio, Y. Oyola, X. Zhu, D.-e. Jiang, S. Dai, Effect of alkyl and aryl substitutions on 1,2,4-triazolium-based ionic liquids for carbon dioxide separation and capture, Rsc Advances 3(12) (2013) 3981-3989.

[301] X. Zhu, C.-L. Do-Thanh, S. Dai, Ionic Liquid-Derived Carbonaceous Adsorbents for CO2 Capture, in: A.-H. Lu, S. Dai (Eds.), Porous Materials for Carbon Dioxide Capture, Springer Berlin Heidelberg, Berlin, Heidelberg, 2014, pp. 1-14. 
[302] V. Gargiulo, M. Alfè, P. Ammendola, F. Raganati, R. Chirone, CO2 sorption on surface-modified carbonaceous support: Probing the influence of the carbon black microporosity and surface polarity, Applied Surface Science 360 (2016) 329-337. [303] X.Q. Wang, Q. Zhu, S.M. Mahurin, C.D. Liang, S. Dai, Preparation of free-standing high quality mesoporous carbon membranes, Carbon 48(2) (2010) 557-560.

[304] S.-H. Chai, P.F. Fulvio, P.C. Hillesheim, Z.-A. Qiao, S.M. Mahurin, S. Dai, "Brickand-mortar" synthesis of free-standing mesoporous carbon nanocomposite membranes as supports of room temperature ionic liquids for $\mathrm{CO} 2-\mathrm{N} 2$ separation, Journal of Membrane Science 468 (2014) 73-80.

[305] T.C. Merkel, H. Lin, X. Wei, R. Baker, Power plant post-combustion carbon dioxide capture: An opportunity for membranes, Journal of Membrane Science 359(1-2) (2010) 126139.

[306] N.P. Wickramaratne, M. Jaroniec, Importance of small micropores in CO2 capture by phenolic resin-based activated carbon spheres, Journal of Materials Chemistry A 1(1) (2013) 112-116.

[307] X. Zhu, S. Chai, C. Tian, P.F. Fulvio, K.S. Han, E.W. Hagaman, G.M. Veith, S.M. Mahurin, S. Brown, H. Liu, S. Dai, Synthesis of Porous, Nitrogen-Doped Adsorption/Diffusion Carbonaceous Membranes for Efficient CO2 Separation, Macromolecular Rapid Communications 34(5) (2013) 452-459.

[308] S.M. Mahurin, P.F. Fulvio, P.C. Hillesheim, K.M. Nelson, G.M. Veith, S. Dai, Directed Synthesis of Nanoporous Carbons from Task-Specific Ionic Liquid Precursors for the Adsorption of CO2, ChemSusChem 7(12) (2014) 3284-3289.

[309] A. Primo, A. Forneli, A. Corma, H. García, From Biomass Wastes to Highly Efficient CO2 Adsorbents: Graphitisation of Chitosan and Alginate Biopolymers, ChemSusChem 5(11) (2012) 2207-2214.

[310] N.P. Wickramaratne, J. Xu, M. Wang, L. Zhu, L. Dai, M. Jaroniec, Nitrogen Enriched Porous Carbon Spheres: Attractive Materials for Supercapacitor Electrodes and CO2 Adsorption, Chemistry of Materials 26(9) (2014) 2820-2828.

[311] J. Liu, N.P. Wickramaratne, S.Z. Qiao, M. Jaroniec, Molecular-based design and emerging applications of nanoporous carbon spheres, Nature Materials 14 (2015) 763. [312] D. Wang, X. Ma, C. Sentorun-Shalaby, C. Song, Development of Carbon-Based "Molecular Basket" Sorbent for CO2 Capture, Industrial \& Engineering Chemistry Research 51(7) (2012) 3048-3057.

[313] H. He, W. Li, M. Zhong, D. Konkolewicz, D. Wu, K. Yaccato, T. Rappold, G. Sugar, N.E. David, K. Matyjaszewski, Reversible CO2 capture with porous polymers using the humidity swing, Energy \& Environmental Science 6(2) (2013) 488-493.

[314] J. Menéndez, A. Arenillas, B. Fidalgo, Y. Fernández, L. Zubizarreta, E.G. Calvo, J.M. Bermúdez, Microwave heating processes involving carbon materials, Fuel Processing Technology 91(1) (2010) 1-8.

[315] S.E. Skrabalak, Ultrasound-assisted synthesis of carbon materials, Physical Chemistry Chemical Physics 11(25) (2009) 4930-4942.

[316] J.N. Coleman, M. Lotya, A. O’Neill, S.D. Bergin, P.J. King, U. Khan, K. Young, A. Gaucher, S. De, R.J. Smith, I.V. Shvets, S.K. Arora, G. Stanton, H.-Y. Kim, K. Lee, G.T. Kim, G.S. Duesberg, T. Hallam, J.J. Boland, J.J. Wang, J.F. Donegan, J.C. Grunlan, G. Moriarty, A. Shmeliov, R.J. Nicholls, J.M. Perkins, E.M. Grieveson, K. Theuwissen, D.W. McComb, P.D. Nellist, V. Nicolosi, Two-Dimensional Nanosheets Produced by Liquid Exfoliation of Layered Materials, Science 331(6017) (2011) 568-571.

[317] Y. Hernandez, V. Nicolosi, M. Lotya, F.M. Blighe, Z.Y. Sun, S. De, I.T. McGovern, B. Holland, M. Byrne, Y.K. Gun'ko, J.J. Boland, P. Niraj, G. Duesberg, S. Krishnamurthy, R. 
Goodhue, J. Hutchison, V. Scardaci, A.C. Ferrari, J.N. Coleman, High-yield production of graphene by liquid-phase exfoliation of graphite, Nature Nanotechnology 3(9) (2008) 563568.

[318] U. Khan, A. O'Neill, M. Lotya, S. De, J.N. Coleman, High-Concentration Solvent Exfoliation of Graphene, Small 6(7) (2010) 864-871.

[319] M. Lotya, Y. Hernandez, P.J. King, R.J. Smith, V. Nicolosi, L.S. Karlsson, F.M. Blighe, S. De, Z.M. Wang, I.T. McGovern, G.S. Duesberg, J.N. Coleman, Liquid Phase Production of Graphene by Exfoliation of Graphite in Surfactant/Water Solutions, Journal of the American Chemical Society 131(10) (2009) 3611-3620.

[320] A. Ciesielski, P. Samorì, Graphene via sonication assisted liquid-phase exfoliation, Chem. Soc. Rev. 43(1) (2014) 381-398.

[321] X. Wang, P.F. Fulvio, G.A. Baker, G.M. Veith, R.R. Unocic, S.M. Mahurin, M. Chi, S. Dai, Direct exfoliation of natural graphite into micrometre size few layers graphene sheets using ionic liquids, Chemical Communications 46(25) (2010) 4487-4489. 


\section{${ }^{*}$ Declaration of Interest Statement}

\section{COLLEGE OF ENGINEERING}

Department of Nuclear Engineering

\section{ATM TEXAS A\&M

The authors declare no financial or competing conflict of interest. All authors have contributed equally to the writing and to the revisions of the manuscript. All authors have approved the final version of the article being submitted to Carbon.

Pasquale F. Fulvio, PhD. Chemistry

E-mail:pfulvio@tamu.edu 